\title{
Contact and Distant Luminescence Quenching in Solutions
}

\author{
Anatoly I. Burshtein \\ Weizmann Institute of Science, Rehovot 76100, Israel \\ Correspondence should be addressed to Anatoly I. Burshtein, cfbursh@wisemail.weizmann.ac.il
}

Received 17 April 2008; Revised 13 June 2008; Accepted 8 July 2008

Recommended by Eric Vauthey

The limitations and advantages of modern encounter theories of remote transfer are discussed, as well as their application to particular transfer reactions assisted by encounter diffusion. Comparison is made with contact multiparticle theories, Brownian dynamic simulations, and the actual experimental data requiring a distant description of energy and/or electron transfer.

Copyright (C) 2009 Anatoly I. Burshtein. This is an open access article distributed under the Creative Commons Attribution License, which permits unrestricted use, distribution, and reproduction in any medium, provided the original work is properly cited.

\section{Introduction}

Non-Markovian chemical kinetics started a century ago based on the famous work of Smoluchowski [1] who studied the encounter diffusion of spherical particles reacting at any contact. The model implied that the reaction is accomplished as soon as the intersphere distance $r$ reduces to the size of their diameter, $\sigma$. A typical example of such a reaction is the irreversible quenching of excited molecule $A^{*}$ by the impurity $B$, stopping abruptly the luminescence of $A^{*}$ :

$$
\begin{gathered}
A^{*}+B \stackrel{k_{A}}{\longrightarrow} A+B \\
\downarrow^{\tau_{A}}
\end{gathered}
$$

The time-dependent reaction constant established by Smoluchowski,

$$
k_{A}(t)=4 \pi \sigma D\left[1+\sqrt{\frac{\sigma^{2}}{\pi D t}}\right],
$$

makes the differential kinetic equation non-Markovian:

$$
\dot{N}_{A}^{*}=-k_{A}(t) c N_{A}^{*}-\frac{N_{A}^{*}}{\tau_{A}} .
$$

It becomes the conventional Markovian equation of chemical kinetics only over long times when $k_{A}(t)$ approaches the constant (stationary) value $\lim _{t \rightarrow \infty} k_{A}(t)=4 \pi \sigma D \equiv k_{D}$. This is the diffusional rate constant linear in encounter diffusion coefficient $D$.

In reality, not any contact of reactants results in complete quenching of $A^{*}$. Being less efficient, such contacts are characterized by a phenomenological parameter $k_{a}$, known as the kinetic rate constant. It was first introduced in the extended contact theory of Collins and Kimball [2] wherein the Markovian (stationary) limit of $k_{A}(t)$ is

$$
k=\lim _{t \rightarrow \infty} k_{A}(t)=\frac{k_{a} k_{D}}{k_{a}+k_{D}} .
$$

However, such a generalization did not overcome the contact nature of the theory that could be scarcely applied to the distant electron transfer reactions and even more so to the long distant energy transfer.

Only an original differential encounter theory (DET) elaborated in the late 1960s overcame the contact limitations of the Smoluchowski-Collins-Kimball model [3-5]. Instead of the kinetic rate constant $k_{a}$, it incorporates into the theory the noncontact, space-dependent rate $W_{A}(r)$. The latter determines $k_{a}$ as well as the whole non-Markovian kinetics of quenching which becomes exponential (Markovian) at later time. The stationary quenching constant $k=4 \pi R_{Q} D$ and its effective quenching radius $R_{Q}$ are also determined by $W_{A}(r)$ and $D$. DET had a tremendous success in the treatment of luminescence quenching by irreversible energy or electron transfer [6]. Assuming an exponential shape of $W(r)$ [7], the theory attempted to fit the real viscosity dependence of the quenching radius $R_{Q}(D)$ [8]. 
Although DET covers only the irreversible transfer, it is known to be exact in the particular case of the target problem (immobile $A^{*}$ quenched by independently moving point $B$ 's) [9]. In the simplest case the time-dependent reaction constant of DET substituted for its contact analog (2) has the following definition:

$$
k_{A}(t)=\int W_{A}(r) v(r, t) e^{t / \tau_{A}} d^{3} r .
$$

Here $\tau_{A}$ is the excitation life time and $\nu(r, t)$ is the pair correlation function of the reactants, obeying the equation for encounter diffusion accompanied by a distant reaction with the rate $W_{A}(r)$ :

$$
\dot{v}=-\left(\frac{1}{\tau_{A}}+W_{A}\right) \nu+D \Delta \nu
$$

At later times, the theory becomes Markovian, that is, $k_{A}(t) \rightarrow k=4 \pi R_{Q} D$ which is the conventional (time independent) rate constant.

At the same time, DET failed to describe the reversible reaction between the meta-stable reactants, $A^{*}$ and $B^{*}$. When their lifetimes are different $\left(\tau_{A} \neq \tau_{B}\right)$, one of the DET "rate constants" diverges. To avoid such a divergency, the alternative integral encounter theory (IET) was developed [10-12] and applied to the reaction of quasiresonance energy transfer [13-15]. Since IET is a kind of memory function formalism, the time-dependent rate constants of DET give way to the memory functions (the kernels of the integral terms) of IET equations not ever turning to the Markovian ones even at later times. On the other hand, the stationary reaction characteristics such as the fluorescence yield or the yields of reaction products are even easier to calculate with IET. The Laplace transformation turns the integral equations into algebraic ones which can be solved analytically. However, IET was known from the very beginning [13-16] as a theory for the lowest concentration of reactants, $N_{A}$ and $N_{B}$. Some of the multiparticle effects accounted for by DET are lost in IET.

To minimize this drawback, a modified version of the integral theory (MET) was developed [17-23]. Creation of MET extended IET to higher concentrations keeping its advantages in accounting for the reaction reversibility but eliminating some of the weaknesses demerits in the asymptotic description of the long time kinetics. The contemporary matrix versions of IET $[14,15]$ and MET [24-26] allow considering reactions of any complexity and arbitrary number of internal electronic states of the reactants and products including their spin states.

In parallel, great progress was made in extending the contact reaction model to higher concentrations of reacting spheres. A number of the multiparticle Kernel theories, MPK1, MPK2, MPK3, were developed [27-29] as well as the superposition approximation [30-33] (SA) and some others. More recently, the relaxation time approximation (RTA) was developed which revives the archaic Smoluchowski approach by accounting for triple encounters: the pair $\mathrm{AB}$ with one more particle B ("bachelor" intervening in the family business). Although original, the self-consistent RTA
(SCRTA) [34] does not add very much to more fundamental multiparticle theories in describing the high concentration effects. Even less warranted are the latest attempts to find better phenomenological constants for RTA, verifying the choice by how it agrees with Brownian dynamic (BD) simulations of reaction kinetics [35].

The principle difference between encounter theories and the inferior Smoluchowski-like approach is in their ability to account for the internal states of the reactants (including spin-multiplicity of the pairs) and the distant dependence of the transfer rates or corresponding Hamiltonian. To emphasize this point this review has the following structure. A brief overview of the integral theory of intra- and intermolecular transfer and later IET modifications is given in Section 2. Also the long time reaction kinetics of contact reactions studied with IET, MET and competing multiparticle theories is compared there with the latest $\mathrm{BD}$ simulations. Then we consider the quantum yields of the luminescence, reversible exciplex formation and electroluminescence (Section 3), focusing attention on their concentration dependence and difference between $\delta$-pulse and stationary excitation. Finally, we turn to the spin assisted reactions (Section 4) and the remote electron transfer (Section 5) using the integral and original unified theory (UT) for fitting the actual experimental data collected in these sections.

\section{Integral Encounter Theories}

Among numerous applications of the integral theory, the reversible reactions of intermolecular or intramolecular energy transfer are the simplest ones. To demonstrate clearly the main features of the integral (memory function) formalism it is better to start from them.

2.1. Intermolecular Energy Transfer. The transfer first studied with IET had the following reaction scheme [13]:

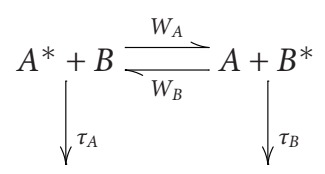

To make the problem even easier let us assume that the nonexcited partners are present in great excess $\left([A]=N_{A} \gg\right.$ $N_{A}^{*}=\left[A^{*}\right]$ and $\left.[B]=N_{B} \gg N_{B}^{*}=\left[B^{*}\right]\right)$. Then the terms in the kinetic equations which are second order in the excited state concentrations, $N_{A}^{*}(t)$ and $N_{B}^{*}(t)$, are negligible and the set of two IET equations can be linearized in the particle densities [15]:

$$
\begin{aligned}
\dot{N}_{A}^{*}= & N_{A} \int_{0}^{t}\langle S(t-\tau)\rangle N_{B}^{*}(\tau) d \tau \\
& -N_{B} \int_{0}^{t}\langle R(t-\tau)\rangle N_{A}^{*}(\tau) d \tau-\frac{N_{A}^{*}}{\tau_{A}}, \\
\dot{N}_{B}^{*}= & -N_{A} \int_{0}^{t}\langle S(t-\tau)\rangle N_{B}^{*}(\tau) d \tau \\
& +N_{B} \int_{0}^{t}\langle R(t-\tau)\rangle N_{A}^{*}(\tau) d \tau-\frac{N_{B}^{*}}{\tau_{B}} .
\end{aligned}
$$


This set should be solved with the initial conditions

$$
N_{A}^{*}(0)=N_{0}, \quad N_{B}^{*}(0)=0,
$$

provided that $A^{*}$ was created by $\delta$-pulse excitation at $t=0$.

In any memory function formalism the main problem is how to define the kernels "memory functions" of integral equations. The great advantage of IET is the absence of such a problem. The space-averaged kernels of the integral equations (8) are defined from first principles by their Laplace transformations:

$$
\begin{aligned}
\langle\widetilde{S}(s)\rangle & =\left(s+\frac{1}{\tau_{B}}\right) \int\left[W_{B}(r) \tilde{\mu}_{3}(r, s)-W_{A}(r) \tilde{\nu}_{3}(r, s)\right] d^{3} r, \\
\langle\widetilde{R}(s)\rangle & =\left(s+\frac{1}{\tau_{A}}\right) \int\left[W_{A}(r) \tilde{\nu}_{2}(r, s)-W_{B}(r) \tilde{\mu}_{2}(r, s)\right] d^{3} r .
\end{aligned}
$$

The auxiliary equations for the dyads of pair distributions $\nu_{3}, \mu_{3}$, and $\nu_{2}, \mu_{2}$ are the same:

$$
\begin{aligned}
& \dot{\nu}=-\left(\frac{1}{\tau_{A}}+W_{A}\right) \nu+W_{B} \mu+D \Delta v, \\
& \dot{\mu}=-\left(\frac{1}{\tau_{B}}+W_{B}\right) \mu+W_{A} \nu+D \Delta \mu .
\end{aligned}
$$

Only the initial conditions for them are different:

$$
\begin{aligned}
& \nu_{2}(r, 0)=1, \\
& \mu_{2}(r, 0)=0 ; \\
& \nu_{3}(r, 0)=0, \\
& \mu_{3}(r, 0)=1 .
\end{aligned}
$$

The global structure of IET formalism is similar to that of DET. Although the kinetic equations are integral, their kernels are defined via the same transfer rates, $W_{A}(r)$ and $W_{B}(r)$, and the pair distribution functions obey the auxiliary equations similar to that of DET. when

It is very easy to solve (11) in the contact approximation

$$
W_{A}(r)=\frac{k_{a} \delta(r-\sigma)}{4 \pi \sigma^{2}}, \quad W_{B}(r)=\frac{k_{b} \delta(r-\sigma)}{4 \pi \sigma^{2}} .
$$

In highly polar solvents,

$$
k_{a}=\int W_{A}(r) d^{3} r, \quad k_{b}=\int W_{B}(r) d^{3} r
$$

are the kinetic rate constants of the forward and backward transfer. They relate both the kernels

$$
\langle\widetilde{R}(s)\rangle=k_{a} \hat{\Sigma}(s), \quad\langle\widetilde{S}(s)\rangle=k_{b} \hat{\Sigma}(s),
$$

to a single factor

$$
\begin{aligned}
\tilde{\Sigma}(s) & =\left[1+\frac{k_{a} / k_{D}}{1+\sqrt{\left(s+1 / \tau_{A}\right) \tau_{d}}}+\frac{k_{b} / k_{D}}{1+\sqrt{\left(s+1 / \tau_{B}\right) \tau_{d}}}\right]^{-1} \\
& =\frac{1}{F(s)}
\end{aligned}
$$

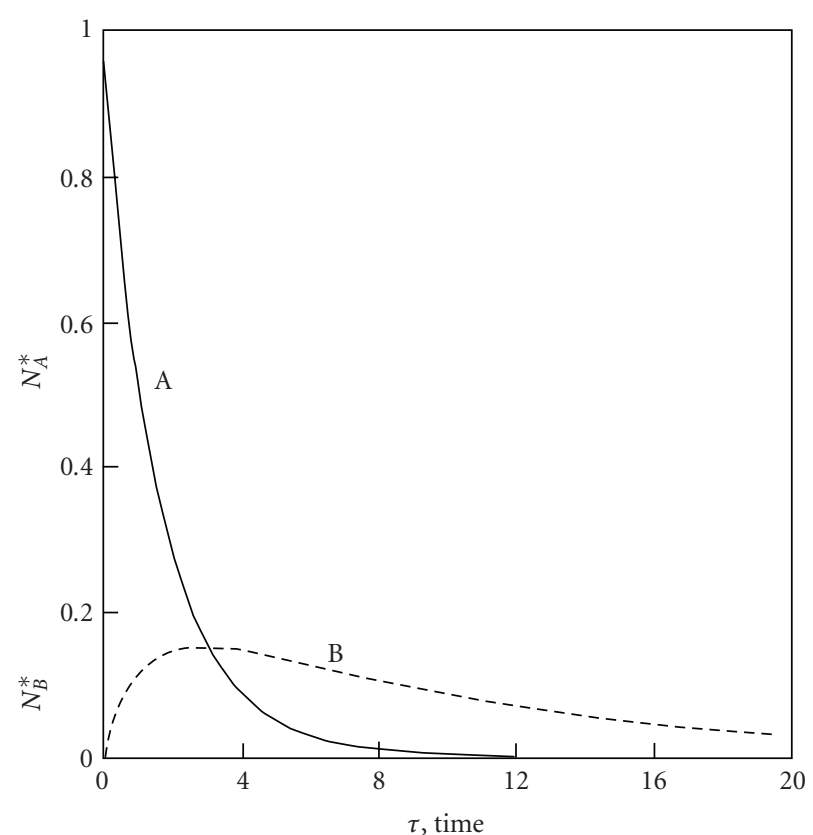

FIgURE 1: The decay of the initially excited molecule, $N_{A}^{*}(t)$ and (A) accumulation/dissipation of transferred energy, $N_{B}^{*}(t)$, (B) reflected receipt and reimbursement of energy by a partner molecule [14].

where $\tau_{d}=\sigma^{2} / D$ is the encounter time. With these kernels, the general IET equations (8) take the particular form specific only to the contact reactions [15]:

$$
\begin{aligned}
\dot{N}_{A}^{*}= & k_{b} N_{A} \int_{0}^{t} \Sigma(t-\tau) N_{B}^{*}(\tau) d \tau \\
& -k_{a} N_{B} \int_{0}^{t} \Sigma(t-\tau) N_{A}^{*}(\tau) d \tau-\frac{N_{A}^{*}}{\tau_{A}}, \\
\dot{N}_{B}^{*}= & -k_{b} N_{A} \int_{0}^{t} \Sigma(t-\tau) N_{B}^{*}(\tau) d \tau \\
& +k_{a} N_{B} \int_{0}^{t} \Sigma(t-\tau) N_{A}^{*}(\tau) d \tau-\frac{N_{B}^{*}}{\tau_{B}} .
\end{aligned}
$$

At a great excess of the $B$ molecule, one may neglect the reencounters of $B^{*}$ with $A$ in the bulk. In this particular case, which attracted the most attention, there is actually only a single $A$ surrounded by $B$ s. In such a situation, the general set (17) reduces to even a simpler one:

$$
\begin{aligned}
& \dot{N}_{A}^{*}-k_{a} N_{B} \int_{0}^{t} \Sigma(t-\tau) N_{A}^{*}(\tau) d \tau-\frac{N_{A}^{*}}{\tau_{A}}, \\
& \dot{N}_{B}^{*}=+k_{a} N_{B} \int_{0}^{t} \Sigma(t-\tau) N_{A}^{*}(\tau) d \tau-\frac{N_{B}^{*}}{\tau_{B}} .
\end{aligned}
$$

These equations describe the quenching of the initially excited $A$, as well as accumulation of energy on $B$, its reverse transfer to $A$, and subsequent natural decay after the end of the encounter (Figure 1). From here on we should discriminate between the qualitatively different situations. 
(1) Energy quenching. This is a case when either the transfer is irreversible $k_{b}=0$ or $\tau_{B} \rightarrow 0$ so that nothing can be transferred backward.

(2) Equal times. In a border case, $\tau_{A}=\tau_{B}=\tau$, the decay of excited states and energy redistribution between them proceed independently.

(3) Energy storing. At $\tau_{A}<\tau_{B}$ the initial quenching of $A^{*}$ by the forward energy transfer results in energy storing by $B$ 's and the backward transfer from them to the short-lived $A^{*}$ during the same encounter.

To describe the energy quenching kinetics one can transform the integral equation (18a) to a differential one, (3), used in DET. This procedure is very delicate, especially since there is a power decay of the memory function. It is valid in a restricted time interval, where the main part of the quenching proceeds quasiexponentially with the timedependent rate constant [14],

$$
k_{A}(t)=k_{a} \int_{0}^{t} \sum\left(t^{\prime}\right) e^{t^{\prime} / \tau_{A}} d t^{\prime},
$$

that monotonously decreases up to the stationary (Markovian) value

$$
k_{s}=\lim _{t \rightarrow \infty} k_{A}=k_{a} \tilde{\Sigma}\left(-\frac{1}{\tau_{A}}\right) .
$$

In fact, this value is unattainable because the reduction of IET to DET does not hold so long. The asymptotic behavior of $N_{A}^{*}$ in IET was shown to be the following [14]:

$$
\frac{N_{A}^{*}(t)}{N_{A}^{*}(0)}= \begin{cases}\exp \left(-c \int_{0}^{t} k_{A}\left(t^{\prime}\right) d t^{\prime}-\frac{t}{\tau_{A}}\right) & \text { at } t \longrightarrow 0, \\ \left(\frac{\tau_{d}}{t}\right)^{3 / 2} \exp \left(-\frac{t}{\tau_{A}}\right) & \text { at } t \longrightarrow \infty .\end{cases}
$$

However, the power time asymptotic quenching in IET was shown to be false at least at $\tau_{B} \leq \tau_{A}$ and was essentially corrected by the multiparticle theories reviewed below.

Qualitatively different are the results in the opposite, energy storing situation when $\tau_{B}>\tau_{A}$. The rate constant (19) changes the sign with time and diverges while the "stationary constant" (20) becomes a complex quantity. In contrast, the effective IET analog of (19),

$$
k_{\mathrm{eff}}(t)=-\frac{d \ln \left(N_{A}^{*} e^{t / \tau_{A}}\right)}{c d t},
$$

can be specified using the solution to the original IET equation (18a). It does not diverge although it becomes negative at long times (Figure 2). This is just a result of changing the sign of the energy flux first directed from $A$ to $B$ but then turning back from $B$ to $A$. In other words, the initial decay of the excited state is faster than the natural one but finally becomes slower and obeys the asymptotic power time dependence over very long times [14]:

$$
\frac{N_{A}^{*}}{N_{0}}=\left(\frac{\tau_{d}}{t}\right)^{3 / 2} e^{-t / \tau_{B}} \quad \text { at } \tau_{A}<\tau_{B} .
$$

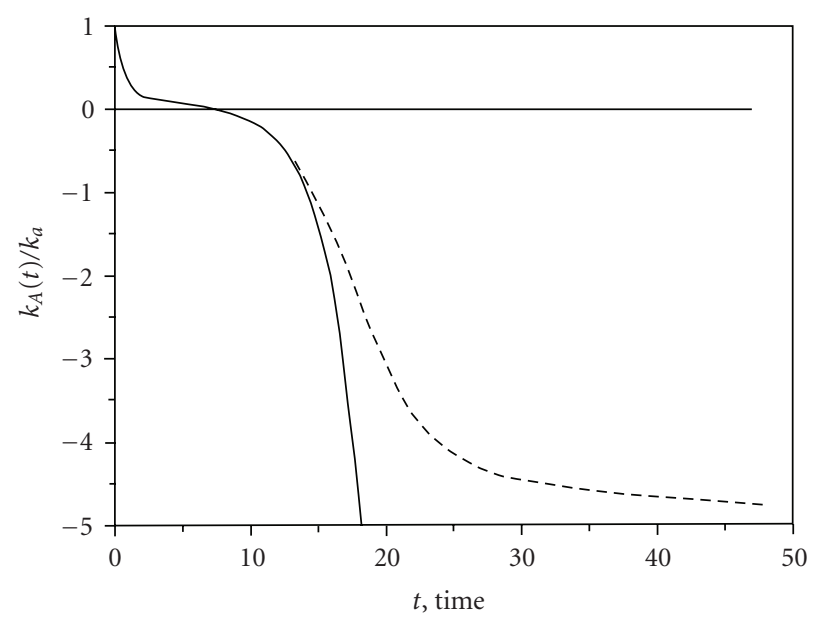

FIGURE 2: The sign alteration of the effective reaction constant of IET (dashed line) and that of DET (solid line), in the case of energy storing (at $\left.\tau_{B}=\infty, \tau_{A}=2 \tau_{d}\right)$ and $k_{a} c \tau_{d}=0.1$ [14].

It should be noted that a similar power law time dependence is an exact result of the geminate reaction of reversible dissociation, $A B \rightleftarrows A+B$, first discovered by Berg [36] and re-derived afterwards [37]. This law was confirmed experimentally [38] and essentially generalized later on [3941].

2.2. Intramolecular Energy Transfer. Even a simpler example of a reversible reaction of the same sort provides the intersystem crossing $A^{*} \rightleftarrows B^{*}$ induced by an inert partner $C$ according to the following scheme:

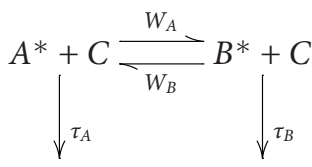

Initially excited $A^{*}$ as well as $B^{*}$ are two electronic states of the same particle $A$ reacting with $C$ given in concentration $c=$ const. In the contact approximation the IET equations take the form [26]

$$
\begin{aligned}
\frac{d N_{A}^{*}}{d t}= & -k_{a} c \int_{0}^{t} \Sigma(t-\tau) N_{A}^{*}(\tau) d \tau \\
& +k_{b} c \int_{0}^{t} \Sigma(t-\tau) N_{B}^{*}(\tau) d \tau-\frac{N_{A}^{*}}{\tau_{A}} \\
\frac{d N_{B}^{*}}{d t}= & k_{a} c \int_{0}^{t} \Sigma(t-\tau) N_{A}^{*}(\tau) d \tau \\
& -k_{b} c \int_{0}^{t} \Sigma(t-\tau) N_{B}^{*}(\tau) d \tau-\frac{N_{B}^{*}}{\tau_{B}},
\end{aligned}
$$

where the Laplace transformation of $\Sigma(t)$ is given by (16). This set of equations is equivalent to the set (17) at $N_{A}=$ $N_{B}=c$ but the reduction to (18a), (18b) is impossible and the backward transfer during reencounters is inevitable.

However, the numerical solution of (25) was an easy matter. This was done in [26] with the initial conditions 
$N_{A}^{*}(0)=N_{0}, N_{B}^{*}(0)=0$ corresponding to the instantaneous ( $\delta$-pulse) excitation of state $A$. The results are shown in Figure 3. In the absence of $C$ there is only a spontaneous exponential decay of $A^{*}$ (solid line in Figure 3(a)). The intramolecular energy transfer to $B^{*}$ modulates this process. If the transfer is irreversible $\left(k_{b}=0\right)$, it only facilitates the decay. This is the energy quenching proceeding exponentially but faster than the natural decay (dashed line in Figure 3(a)). At $k_{b} \neq 0$, the energy comes back due to reencounters with $C$, supporting the delayed fluorescence from $A^{*}$ if $\tau_{B}>\tau_{A}$. Under such a condition, the dashed-dotted line representing $N_{A}^{*}(t)$ short after rapid quenching goes down much slower than the spontaneous decay. The smaller $k_{b}$, the later, weaker and slower should be the delayed fluorescence. This energy storing is the most pronounced at $\tau_{B}=\infty$.

This picture is supplemented with the corresponding kinetics of energy accumulation in $B^{*}$ and the subsequent decay of this state. The latter takes place even at $\tau_{B}=\infty$ due to the collision induced reverse transfer to $A^{*}$ (Figure 3(b)). Such a decay is absent only at $k_{b}=0$ and is faster, the higher is $k_{b}$. At any $\tau_{A}<\tau_{B}$, the energy is stored in a more stable excited state $B^{*}$ but finally comes back to the short-lived excited state $A^{*}$, causing the delayed fluorescence.

Between the opposite limits of energy quenching $\left(\tau_{A}>\right.$ $\left.\tau_{B}\right)$ and energy storing $\left(\tau_{A}<\tau_{B}\right)$ there is a border case of equal times that deserves special attention. This exceptional case allows the exact multiparticle solution of the problem obtained in [42]. When $\tau_{A}=\tau_{B}=\tau$, the natural decay of both states can be excluded from consideration by a simple substitution:

$$
N_{A}^{*}(t)=\mathcal{P}_{A}(t) e^{-t / \tau}, \quad N_{B}^{*}(t)=\mathcal{P}_{B}(t) e^{-t / \tau} .
$$

The relative populations of excited states, $\mathcal{P}_{A}(t)$ and $\mathcal{P}_{B}(t)$, tend to their equilibrium values as $t \rightarrow \infty$ :

$$
\mathcal{P}_{A}(\infty)=\frac{1}{1+K}=P_{A}, \quad \mathcal{P}_{B}(\infty)=\frac{K}{1+K}=P_{B},
$$

where $K=k_{a} / k_{b}$ is the equilibrium constant in a system of two excited states. The general solution of the problem can be represented as

$$
\mathcal{P}_{A}(t)=\frac{1}{1+K}+\frac{K}{1+K} \mathcal{P}_{\mathrm{eff}}(t)=1-\mathcal{P}_{B}(t),
$$

where $\mathcal{P}_{\text {eff }}(t)$ was the subject of calculations in [42]. After the exact averaging over the multiparticle distribution of $C$ it was shown to be

$$
\mathcal{P}_{\text {eff }}(t)=\exp \left(-c \int_{0}^{t} k\left(t^{\prime}\right) d t^{\prime}\right)
$$

with $k(t)$ obeying the original DET equations (5) and (6) but with substituting $W_{A}(r)+W_{B}(r)$ for $W_{A}(r)$. In the contact approximation this leads to the conventional SmoluchowskiCollins-Kimball solution but with $k_{a}+k_{b}$ substituted for $k_{a}$.

2.3. Intermolecular Electron Transfer. Let us now turn to the reversible reaction of electron transfer, $A^{*}+D \rightleftarrows A^{-}+$ $D^{+}$accompanied by charge recombination. After charge separation, the free ions recombine in the bulk to either the ground state or backward to the excited neutral products, which contribute again to the total fluorescence:

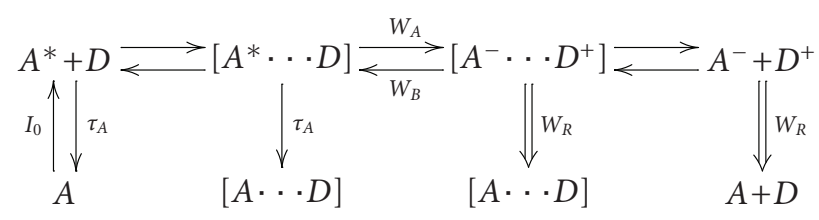

Such a complete scheme enables studying the luminescence quenching proceeding from left to right, as well as the electroluminescence (resulting from the recombination of injected ions) going from right to left.

The complex kinetics of the luminescence switched by $\delta$ excitation of $A$ in reaction (30) was studied in [43] using the general IET equations:

$$
\begin{aligned}
\dot{N}_{A}^{*}= & -c \int_{0}^{t} R^{*}(\tau) N_{A}^{*}(t-\tau) d \tau \\
& +\int_{0}^{t} R^{\sharp}(\tau) P^{2}(t-\tau) d \tau-\frac{N_{A}^{*}(t)}{\tau_{A}}+I_{0} N_{A}, \\
\dot{P}= & c \int_{0}^{t} R^{\dagger}(\tau) N_{A}^{*}(t-\tau) d \tau-\int_{0}^{t} R^{\ddagger}(\tau) P^{2}(t-\tau) d \tau,
\end{aligned}
$$

where $P(t)=\left[A^{-}\right]=\left[D^{+}\right]$is the free-ion concentration and $c=[D]=$ const is the concentration of donors present in great excess. When the geminate recombination is completed and gives way to ion recombination, the latter restores either the ground or excited state of the neutral reactants. The fluorescence of the excited ones is delayed. In fact this is an electroluminescence though not from injected ions but from those which escaped the geminate recombination after photo separation. The density of restored excitations at a later stage of their decay is quadratic in the free-ion concentration that should be large enough to make the delayed fluorescence detectable. To reach this goal one has to use as strong pumping as possible and choose the fluorophors with rather long-lived excited states. Then their fluorescence at times $t>\tau_{A}$ will be stronger than in the absence of charge recombination.

The long time asymptote of the delayed decay can be described by the reduced equation (31), where the light pumping is absent and charge recombination is considered as irreversible in view of a negligible concentration of restored $A^{*}[43]$ :

$$
\begin{aligned}
\dot{N}_{A}^{*}= & -\frac{N_{A}^{*}}{\tau_{A}}-c \int_{0}^{t} R^{*}(\tau) N_{A}^{*}(t-\tau) d \tau \\
& +\int_{0}^{t} R^{\sharp}(\tau) P^{2}(t-\tau) d \tau, \\
\dot{P}= & -\int_{0}^{t} R^{\ddagger}(\tau) P^{2}(t-\tau) d \tau .
\end{aligned}
$$




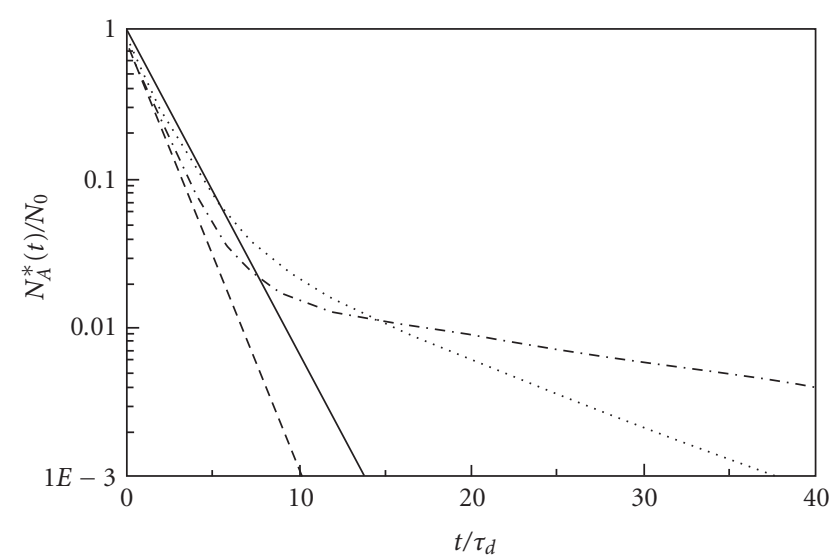

(a)

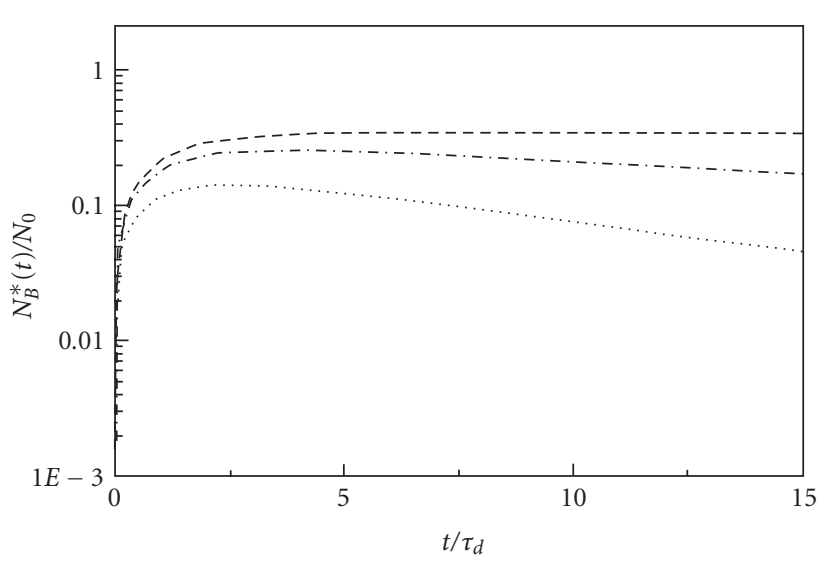

(b)

Figure 3: Relaxation of state populations after instantaneous excitation of $A$ at $\tau_{B}=\infty$ and $c k_{a} \tau_{d}=1.0$. (a) Spontaneous (exponential) decay of $A^{*}$ with lifetime $\tau_{A}=2 \tau_{d}$ (thick straight line) and its dissipation accompanied by intersystem crossing with the backward rates $k_{b}=5.0 k_{D}$ (dotted line), $k_{b}=k_{D}$ (dashed-dotted line), and $k_{b}=0$ (dashed line). (b) Accumulation and dissipation of the initially empty state $B^{*}$, which decays through $A^{*}$ faster the higher is $k_{b}$, or does not decay at all if $k_{b}=0$ [26].

The initial condition for free ions is given by the following equation:

$$
P(0)=N_{A}^{*}(0)(1-\eta) \bar{\varphi}=P_{0},
$$

where the yield of photo-generated ions is $1-\eta$ and the yield of their separation $\bar{\varphi}$ is defined in (67).

Since ions are stable particles, those terms in the integral equations (32) which describe the ion recombination may be transformed at $t \gg \tau_{d}$ into their differential and even Markovian analogs [13]. The same can be done with the remaining ionization term, but at a much longer time, $t \gg \tau_{A}$, when the concentration of excitations levels decreases slowly following charge recombination. Hence, the delayed fluorescence can be described by the following set of differential (Markovian) equations:

$$
\begin{gathered}
\dot{N}_{A}^{*}=-\left[\frac{1}{\tau_{A}}+c \kappa_{0}\right] N_{A}^{*}(t)+k^{*} P^{2}, \\
\dot{P}=-k^{\times} P^{2},
\end{gathered}
$$

where $k^{*}=\widetilde{R}^{\sharp}(0)$ and $k^{\times}=\widetilde{R}^{\ddagger}(0)$. The delayed fluorescence can be obtained from the quasistationary solution of (34):

$$
\begin{aligned}
N_{A}^{*}(t) & =\frac{k^{*} P^{2}}{\left[1 / \tau_{A}+c \kappa_{0}\right]} \longrightarrow \frac{A}{t^{2}}, \\
P & =\frac{P_{0}}{1+k^{\times} P_{0} t} \longrightarrow \frac{1}{k^{\times} t},
\end{aligned}
$$

where

$$
A=\frac{k^{*}}{\left[1 / \tau_{A}+c \kappa_{0}\right]\left(k^{\times}\right)^{2}}
$$

was calculated in the contact approximation in [43]. In the same paper the full kinetics of luminescence was shown to have a long tail approaching the delayed fluorescence asymptote (35a) (Figure 4). The latter screens the intermediate asymptote of the multiparticle quenching (21) which is known to be false in IET.

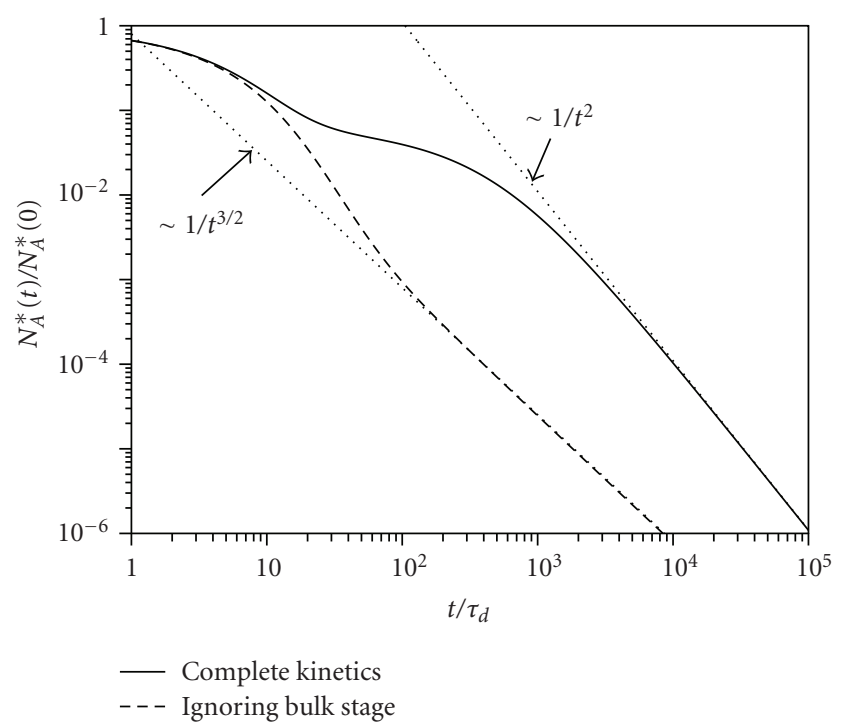

FIGURE 4: The false asymptote of geminate excitation quenching (long dashed line) in comparison to the second power asymptote (dashed line) of true excitation decay (solid line) [43].

2.4. Modifications of Integral Theory. Unfortunately, the IET is the theory keeping only the lowest order terms in reactant concentrations. To account for the higher ones by MET, one only has to modify the kernels of the integral equations. In $[24,25]$ this was recommended to be done by the substitution of the generalized decay rates

$$
\frac{1}{\tau_{A}^{*}}=\frac{1}{\tau_{A}}+k_{a} N_{B} \tilde{\Sigma}(s), \quad \frac{1}{\tau_{B}^{*}}=\frac{1}{\tau_{B}}+k_{b} N_{A} \tilde{\Sigma}(s),
$$

for the inverse times $1 / \tau_{A}$ and $1 / \tau_{B}$ figuring in (16). Such an obtained MET was shown to correct IET even better than the first multiparticle kernel (MPK1) theory [27], which at first corrected the false IET asymptote of quenching. However, 
the authors of the MPK1 intuitively neglected some threeparticle correlations in comparison with others, while MET accounts explicitly for all of them $[24,25]$. An excellent analysis of the drawback in MPK1 and its consequences was recently presented by Ivanov [44]. Fortunately this drawback was later overcome by the same authors [29] making their last theory, MPK3, almost equivalent to MET [25].

MET essentially corrects IET over long times [44]. Figure 5 demonstrates the difference in time behavior between IET and MET effective quenching constants for the forward and reverse transfer,

$$
\begin{aligned}
& k_{f}^{\mathrm{eff}}=\frac{k_{a} \int_{0}^{t} \Sigma(t-\tau) N_{A}^{*}(\tau) d \tau}{N_{A}^{*}(t)}, \\
& k_{r}^{\mathrm{eff}}=\frac{k_{b} \int_{0}^{t} \Sigma(t-\tau) N_{B}^{*}(\tau) d \tau}{N_{B}^{*}(t)} .
\end{aligned}
$$

The former is the analog of (22) which is known from IET to become negative with time (Figures 2 and 5(a)) but in MET turns to be positive again at $t \gg \tau_{d}$ ) (Figure 5(b)). At these long times the transfer proceeds not between geminate partners that have already separated but with many other B's that, being in the ground state, act as the fresh quenchers. As to the quenching constant for stable excitation $B^{*}$, it remains positive at all times in both theories but only in MET approaches the truly stationary value $k_{b} \Sigma(0)$.

In the case of irreversible quenching $\left(k_{b}=0\right)$, the kernel modification can also be done as was recommended earlier [19-23], by the straightforward substitution

$$
\Sigma(t) \longrightarrow \Sigma(t) \exp (-c k t)
$$

where $k$ is the stationary (Markovian) constant of the irreversible transfer (4). The difference between the two recipes of modification originates from the way in which the "point encounter approximation" was used to simplify the three-particle terms; only in space or also in the time domain [24]. However, this choice rather insignificantly affects the results. Performing point encounter approximation in the coordinate space one implies that the reaction pair evolution proceeds at distances larger than the reaction zone which can be ignored (see [24, Equations (6.15) and (6.16)]). This allows to derive the binary kinetic equation by the simplification of three-particle terms in concentration expansion keeping only their dependence on relative mobility of the reactants.

An original matrix reformulation of IET and its modification similar to (39) were presented in [45]. Such an obtained MET is applicable to the reversible reactions of any complexity and is in fact identical to that used in [17-21]. However, the authors did not restrict themselves to the integral form of the theory but transformed their matrix integrodifferential equation into a set of two coupled differential equations which can be solved easier numerically. They also developed a general computer code (available on the Internet [45]) and demonstrated it application to the well-known Lotka-Volterra reaction which is oscillatory with time [46].
2.5. Asymptotic Excitation Decay. The pre-exponential power time dependence of $N_{A}^{*}(t) / N_{A}^{*}(0)$ indicated in (21) is known to be the false IET asymptote for the long time quenching. The latter can be easily seen in the case of irreversible transfer, rather than in the reversible case.

Assuming that the transfer from $A^{*}$ to $B$ is irreversible $\left(k_{b}=0\right)$, the quenching kinetics was studied in [45] by solving the original differential equations equivalent to either IET or MET. As can be seen from Figure 6, the difference between the curves representing these theories is insignificant within the validity limits for IET established in $[17,18]$ :

$$
t \ll \ln \left(\xi^{-1}\right) / k c
$$

where $\xi=(4 / 3) \pi \sigma^{3} c$. The validity region for MET is known to be much wider:

$$
t \ll \frac{\xi^{-1}}{k c}
$$

This was confirmed in [45] by a straightforward comparison of the MET solution with that of DET:

$$
P_{A}^{*}=N_{A}^{*} \exp \left(\frac{t}{\tau_{A}}\right)=\exp \left[-c \int_{0}^{t} k_{A}\left(t^{\prime}\right) d t^{\prime}\right]
$$

The latter which is exact for the target problem is not distinguishable from MET, represented by the solid line in Figure 6. The difference appears to be less than the precision of the numerical calculations, provided $P^{*}(t)>e^{-1 / \xi}$, where $\xi<1$. On the contrary, the dashed curve representing IET significantly deviates from the exact result when the time exceeds the border (40) indicated by the vertical line in this figure. The false asymptote of IET is to the right of it. In other words the IET is valid until $P^{*}(t)>\xi$.

As has been reported, the reversible intramolecular transfer between the meta-stable states

$$
\begin{gathered}
A^{*}+B \stackrel{k_{a}}{\rightleftharpoons} C_{k_{b}}^{*}+D \\
\downarrow_{k_{A}}
\end{gathered}
$$

results in either energy quenching, if $k_{C}>k_{A}$, or in energy storing, if $k_{A}>k_{C}$. However, inspecting the accuracy of different ABCD theories, one cannot use DET as a standard because this theory is not applicable to the energy storing limit (due to the divergency of the time-dependent forward rate constant) [13-16]. The only alternative is to compare the theoretical results with those obtained by the BD simulation of the transfer kinetics.

Since these simulations are always done only for contact reactions between chemically isotropic hard spheres, they can be compared only with contact IET and MET, as well as with MPK, SA, SCRTA, and others, applied to the same Smoluchowsci-like model. Even though in such a primitive model only the concentration and time dependence of $N_{A}^{*}$ can be inspected it is yet useful and instructive. The numerous modifications of SCRTA were the main interest of the authors of the last $\mathrm{BD}$ simulations but some other 


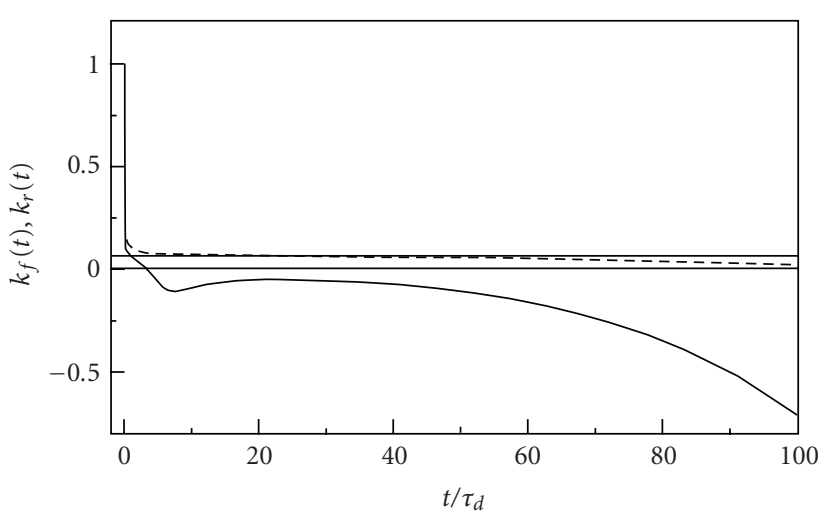

(a)

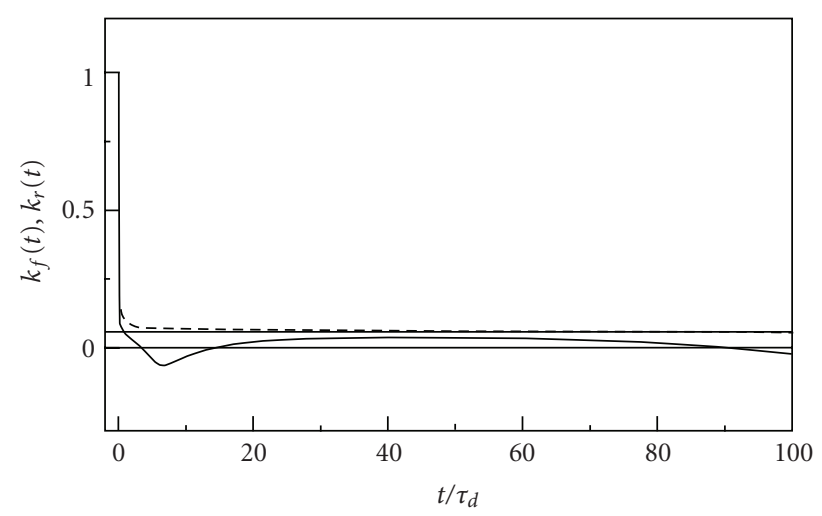

(b)

FIGURE 5: The time effective rate "constants" for the forward and reverse transfer, $k_{f}(t)=k_{f}^{\text {eff }} / k_{a}$ (solid lines) and $k_{r}(t)=k_{r}^{\text {eff }} / k_{a}($ dashed lines), calculated with $\operatorname{IET}(\mathrm{a})$ and MET (b) at $\tau_{B}=\infty$, equal concentrations of $A$ and $B$ and equal kinetic constants $k_{f}^{\text {eff }}(0)=k_{r}^{\text {eff }}(0)=k_{a}=$ $10 k_{D}$. The thin solid line shows the stationary value of $k_{r}^{\text {eff }}(\infty)[44]$.

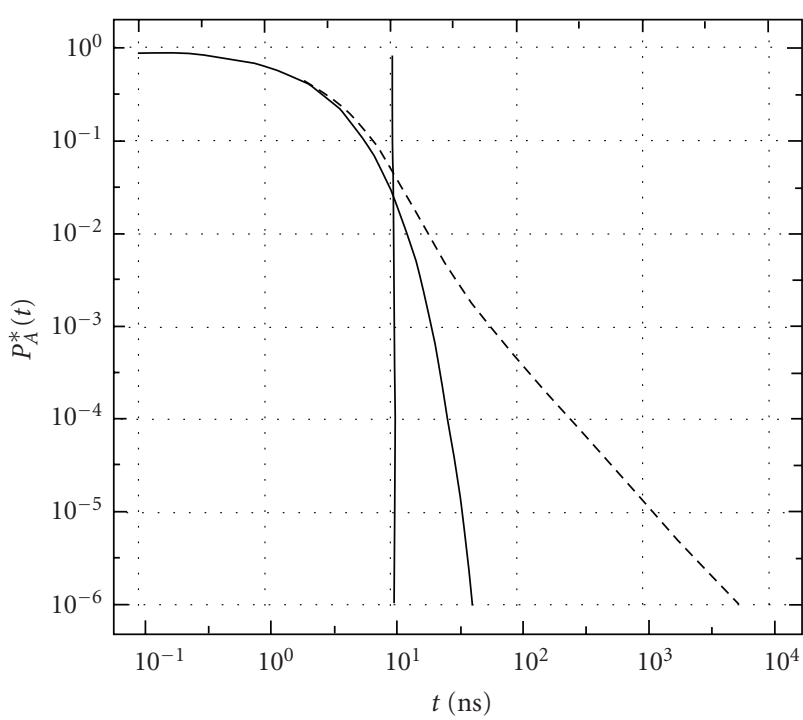

FIGURE 6: The quenching kinetics obtained with IET (dashed line) and MET (solid line). The vertical strip denotes the upper boundary of the region of IET validity [45].

theories were also included into comparison shown in Figure 7 [35]. The two upper panels are represented as they were published (in black and white), while the lower ones are just the colored originals of them placed at our disposal by one of the authors.

We can see from this figure that the two variants of BD simulations, depicted by the open symbols: triangles (algorithm 1) and circles (algorithm 2), are actually indistinguishable. It can also be seen from the black and white panels that they agree perfectly with MPK3 and SCRTA but not as well as with MPK1 and even worse with IET. The black and white presentations of data are suitable to give the impression that IET is the worst among the other theories. To refute this conclusion it is enough to glance at the colored version of the same data.

IET deviates from the exact results only at a high concentration (right panels) where it is known to be inapplicable. In this sense, it is actually "the inferior to the MET" as stated in [35] but only in the same sense as the theory of ideal gases is the inferior to the Van der Waals theory. Moreover, even at large concentrations IET only fails to describe the energy quenching, $k_{C}=10\left(k_{A}=\right.$ $0)$, but not the energy-storing case, $k_{A}=10\left(k_{C}=0\right)$, where the false IET asymptote is screened (as in Figure 4) by delayed fluorescence. Of course, the coincidence of the IET results with $\mathrm{BD}$ simulations is excellent in any case when the concentration is low. Moreover, the expected imperfection of IET at high concentration is totally corrected by implication of MET $\equiv$ MPK3.

In short, the false asymptote of IET comes to light only at high concentrations and only in the energy quenching case. This drawback of IET is completely removed by MET which accounts for the transfer not only to the partner in a pair but also to other surrounding reactants. By this way, MET corrects the high concentration behavior of all the quantum yields considered in Section 3.

\section{Concentration Phenomena}

3.1. Luminescence after Pulse Excitation. The quantum yield of the fluorescence following pulse excitation is

$$
\eta=\frac{\int_{0}^{\infty} N_{A}^{*}(t) d t}{N_{A}^{*}(0) \tau_{A}}
$$

provided that the light excites instantaneously (at $t=0$ ) only $A$ and that the luminescence comes only from this 

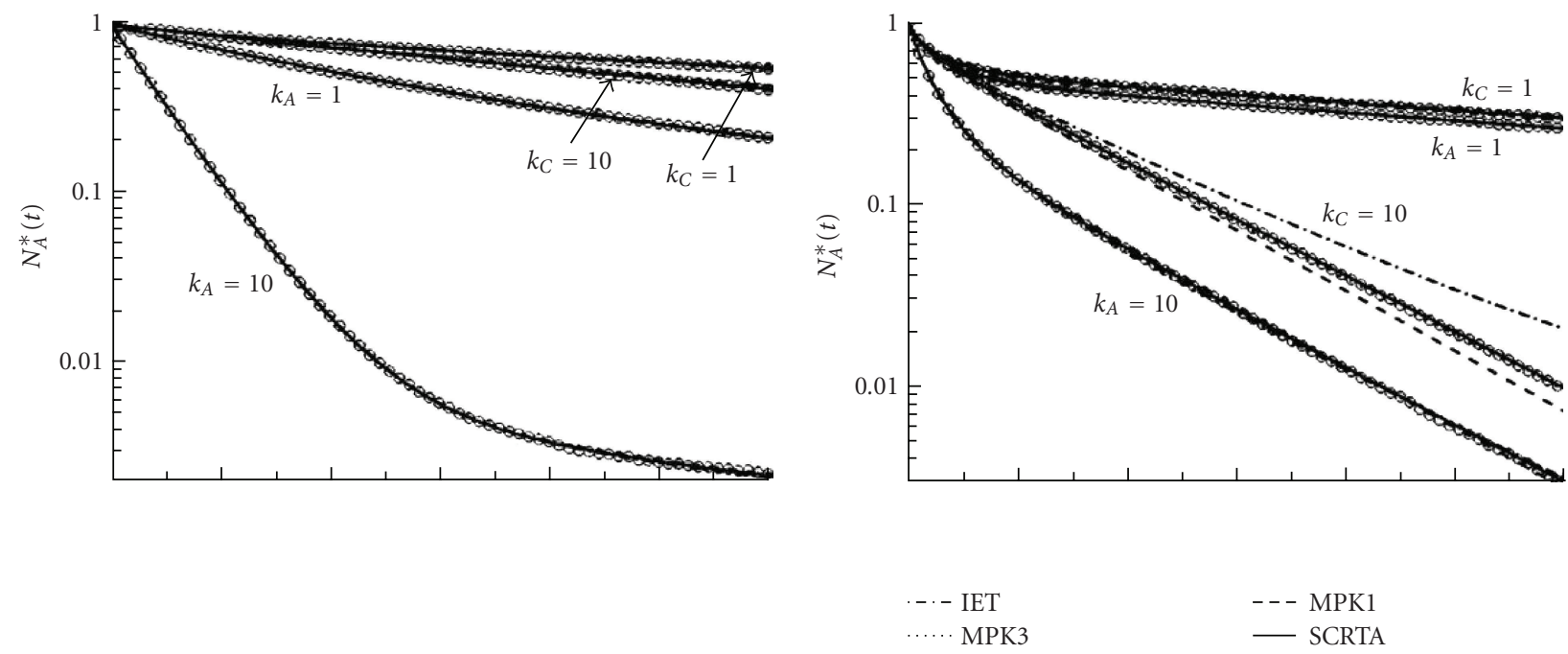

(a)

(b)
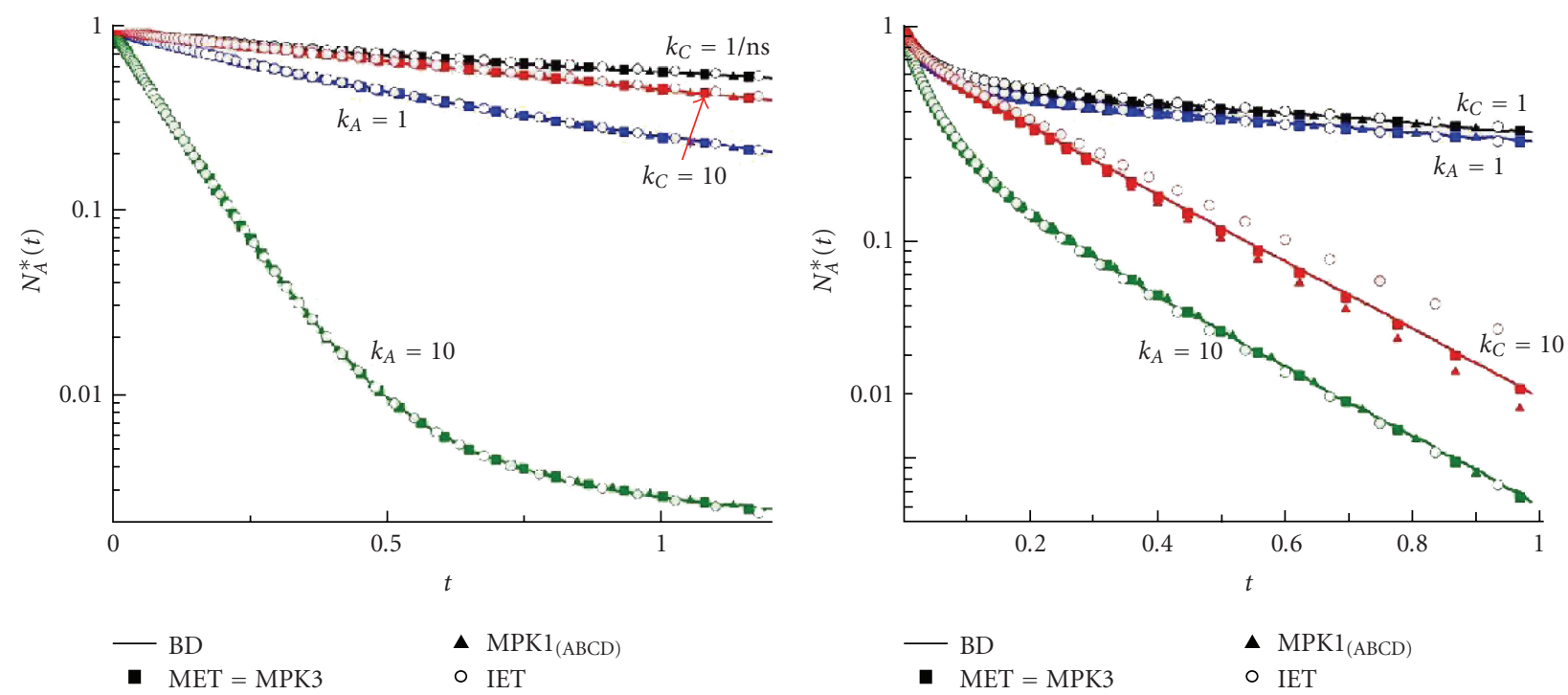

(c)

(d)

Figure 7: The time dependence of the survival probability, $N_{A}^{*}(t)$, of initially excited $A^{*}$ for several values of the unimolecular decay constants, $k_{A}$ and $k_{C}$ (in $\mathrm{ns}^{-1}$, one is zero and the other is indicated in the figure). The parameters are the same everywhere, except that the concentrations in the left panels are low $\left(c_{B}=c_{D}=10^{-3} \AA^{-4}\right)$ being large in the right ones $\left(c_{B}=c_{D}=10^{-3} \AA^{-3}\right)$. In the colored panels, the $\mathrm{BD}$ simulations are presented by solid lines, while all the rest are depicted by symbols indicated in these panels.

particle. The concentration dependence of the yield is always represented by the Stern-Volmer law

$$
\eta=\frac{\tilde{N}_{A}^{*}(0)}{N_{0} \tau_{A}}=\frac{1}{1+N_{B} k_{q} \tau_{A}},
$$

where $k_{q}$ is the quenching constant which is to be investigated.

Let us start doing this from the reversible energy transfer studied in Section 2.1. Using the Laplace transformation of the IET equations (17), one can easily find from (45) that

$$
k_{q}=\frac{K_{A B}}{1+K_{B A} N_{A} \tau_{B}} .
$$

Here $K_{A B}=k_{a} \tilde{\Sigma}(0)$ and $K_{B A}=k_{b} \tilde{\Sigma}(0)$, that is,

$$
\begin{aligned}
\frac{K_{A B}}{k_{a}} & =\frac{1}{1+\left(k_{a} / k_{D}\right) /\left(1+\sqrt{\tau_{d} / \tau_{A}}\right)+\left(k_{b} / k_{D}\right) /\left(1+\sqrt{\tau_{d} / \tau_{B}}\right)} \\
& =\frac{K_{B A}}{k_{b}} .
\end{aligned}
$$

This result obtained by means of IET is concentrationindependent but known to be valid at only the lowest concentrations of $A$ and $B$ [13].

3.2. Irreversible Geminate Reactions. To demonstrate how DET and MET, as well as other multiparticle theories, correct 
this result at higher concentrations, let us focus upon the irreversible transfer setting

$$
K_{B A}=k_{b}=0, \quad N_{B}=c .
$$

In such a case, the theory becomes universal that is equally good for any irreversible quenching, including the parallel transfer of energy and electron [47]

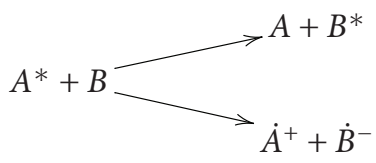

or the double-channel electron transfer studied in $[48,49]$ :

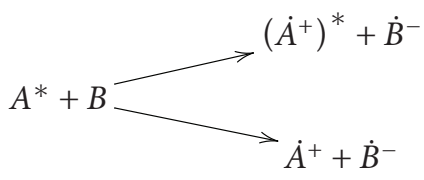

All of them can be briefly represented by the unified scheme of the irreversible energy quenching: $A^{*}+B \rightarrow A+B$.

The quantum yield of the irreversible quenching obeys the Stern-Volmer dependence on concentration $c=N_{B}$ represented in (45). As in the original Stern-Volmer law, the quenching constant obtained from (46) and (47) is concentration-independent:

$$
k_{q}=\frac{k_{a}}{1+k_{a} / k_{D}\left(1+\sqrt{\tau_{d} / \tau_{A}}\right)}=\kappa_{i}
$$

However, contrary to $\kappa_{i}$, the experimentally found $k_{q} \equiv \kappa$ is concentration-dependent and this is a challenge for the theory to find out how the true Stern-Volmer constant $\kappa(c)$ differs from its IET analog $\kappa_{i}=\lim _{c \rightarrow 0} \kappa(c)$.

MET solves this problem substituting $\tau_{A}$ in $(51)$ by $\tau_{A}^{*}(0)$ from (37):

$$
\kappa=\frac{k_{a}}{1+k_{a} / k_{D}\left(1+\sqrt{\left(\tau_{d} / \tau_{A}\right)\left[1+c \kappa_{i} \tau_{A}\right]}\right)} .
$$

In general, this $\kappa$ is concentration-dependent unless the transfer is strongly under kinetic control when $\kappa=k_{a} \ll k_{D}$. In the alternative limit of diffusional transfer, MET gives

$$
\kappa=k_{D}\left(1+\sqrt{\frac{\tau_{d}}{\tau_{A}}\left[1+c \kappa_{i} \tau_{A}\right]}\right)
$$

In [50] this result was compared with that of IET and other theories: SA [30-33] and DET (Figure 8). The Stern-Volmer constant of MET and SA differs a bit from the exact result represented by DET but all of them except IET increase with

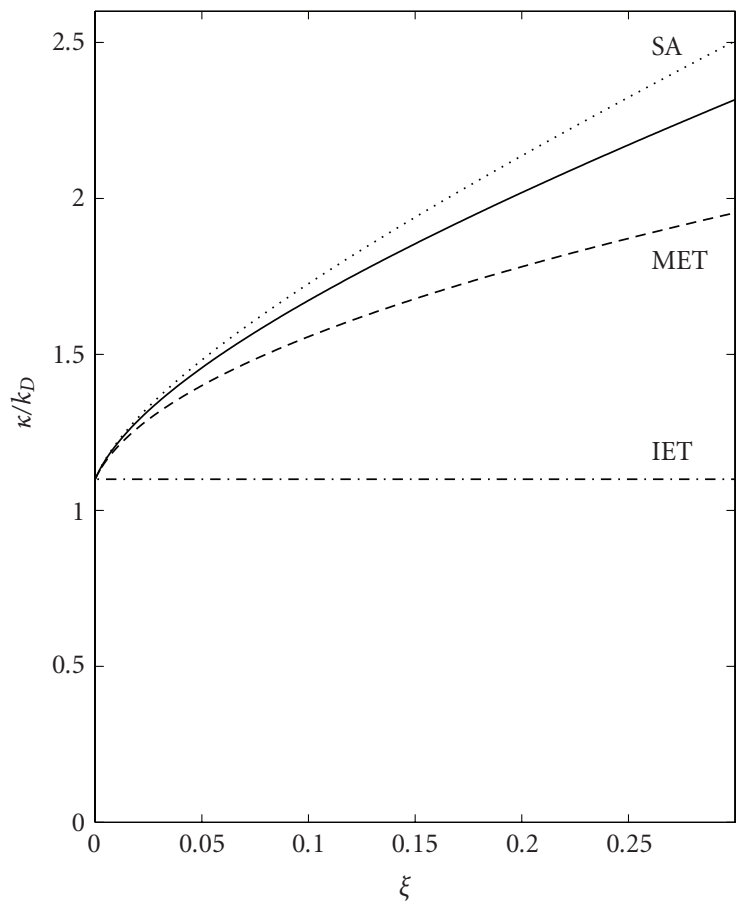

Figure 8: The Stern-Volmer constants as functions of the dimensionless concentration $\xi=4 \pi \sigma c^{3} / 3$ obtained in the contact approximation and under diffusional control at $\tau_{d} / \tau_{A}=0.01$. The thick line represents DET which is exact for the target problem (immobile donors and independently moving acceptors). The rest of the curves are obtained with SA, MET, and IET [50].

the dimensionless concentration $\xi=c v\left(v=(4 / 3) \pi \sigma^{3}\right)$. At moderate concentrations this dependence is always linear,

$$
\frac{\kappa}{k_{D}}=\frac{\kappa}{\kappa_{i}}+A \xi=1+\sqrt{\frac{\tau_{d}}{\tau_{A}}}+A \xi,
$$

but the slope of it appears to be different. For MET obtained by modification (37), $A=(3 / 2) \sqrt{\tau_{A} / \tau_{d}}+3 / 2$ while in the old MET originated from another modification, (39), $A=$ $(3 / 2) \sqrt{\tau_{A} / \tau_{d}}$. The former coincides with that of SA while the latter (shown in figure) is a bit smaller. The true DET value is in between $A=(3 / 2) \sqrt{\tau_{A} / \tau_{d}}+3(1-2 / \pi)$ [50]. Since the concentration correction in (54) is only actual for large $\tau_{A} \gg \tau_{d}$, the constant term in $A$ is insignificant. In the opposite case, the whole correction is negligible compared to the preceding IET term. Hence the difference between the concentration corrections at small $\xi$ is not essential.

However, the comparison of all the theories (of contact multiparticle quenching by point particles $B$ ) was done later for any $\xi$ (Figure 9) [47]. Theoretically, $\kappa$ increases with concentration from the IET value $\kappa_{i}$ up to the kinetic rate constant $k_{0} \equiv k_{a}$, though experimentally available is only the lower (left) part of the graph, $c v<1$. Contact MET, which is identical to MPK3 developed later [29], underestimates the exact $\kappa(c)$ represented by DET. The latter is equivalent to the irreversible version of the first multiparticle kernel theory, MPK1 [27]. The intermediate version of this theory [28], MPK2, as well as the latest model theory SCRTA [34], 


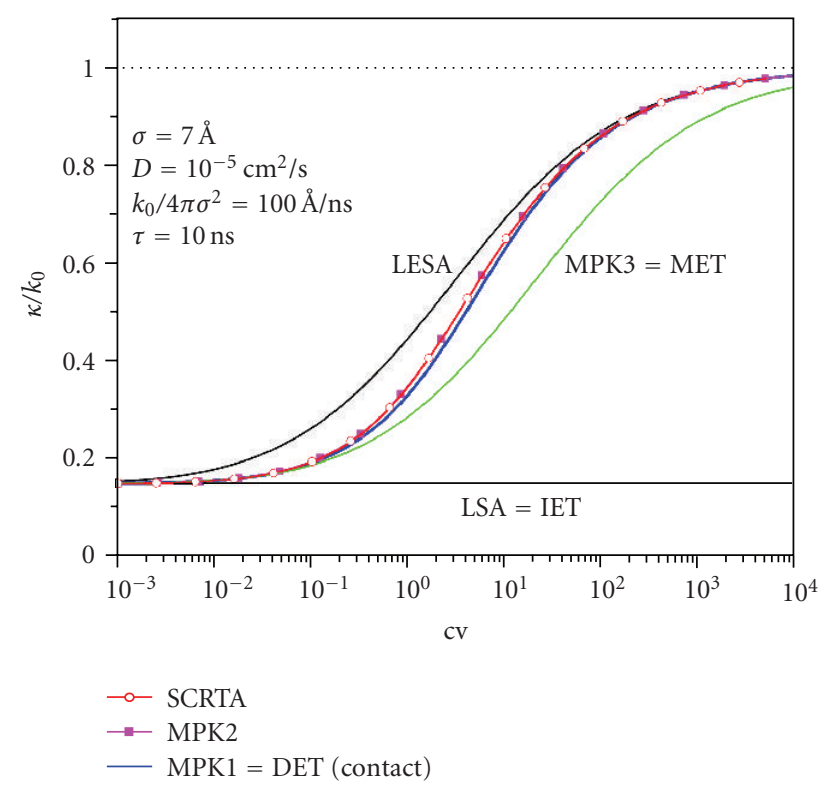

Figure 9: The concentration dependence of the irreversible quenching Stern-Volmer constant $\kappa$ in units of $k_{0} \equiv k_{a}$ for a number of contact theories, provided $k_{a}=3.7 \cdot 10^{10} \mathrm{M}^{-1} \mathrm{~s}^{-1}$ is the same for all of them [47] and [48, Figure 3.88].

almost coincide with DET $=$ MPK1 (irreversible), unlike the linearized extended superposition approximation [51] (LESA) which overestimates $\kappa(c)$.

3.3. IET of Reversible Geminate Reaction. Let us now turn to the geminate reaction similar to that included in scheme (49) but carried out by reversible electron transfer:

$$
\begin{aligned}
& A^{*}+D \underset{W_{B}}{\stackrel{W_{A}}{\gtrless}}\left[A^{-} \ldots D^{+}\right] \stackrel{\bar{\varphi}}{\longrightarrow} A^{-}+D^{+} \\
& I \uparrow \tau_{A} \quad W_{B} \quad \Downarrow W_{R} \\
& \text { A } \\
& {[A \cdots D]}
\end{aligned}
$$

Here $D$ is the donor of an electron, while $A$ is its acceptor. The excitation of $A$ by the short light pulse, $I(t)$, resulting in charge separation, produces the free ions, $D^{+}$and $A^{-}$, with the yield $\phi$. This is the fraction $\bar{\varphi}$ of ions initially born in amount $1-\eta$ but separated escaping recombination to the ground state with the rate $W_{R}$ and the backward electron transfer to excited products with the rate $W_{B}$.

If $A$ 's are present in very low concentration, the density of the free ions is small as well, so that their recombination in the bulk is negligible during a bounded time domain comparable with encounter time $\tau_{d} \sim \sigma^{2} / D$. The IET equations (31) can be reduced for this case by omitting the bulk terms quadratic in $P$, as well as the pumping term:

$$
\begin{gathered}
\dot{N}_{A}^{*}=-c \int_{0}^{t} R^{*}(\tau) N_{A}^{*}(t-\tau) d \tau-\frac{N_{A}^{*}(t)}{\tau_{A}}, \\
\dot{P}=c \int_{0}^{t} R^{\dagger}(\tau) N_{A}^{*}(t-\tau) d \tau .
\end{gathered}
$$

Instead of the pumping term one has to add the initial conditions for these equations which represent the instantaneous excitation of $A$ :

$$
N_{A}^{*}(0)=N_{0}, \quad P(0)=0 .
$$

The luminescence of the pulse excited $A^{*}$ quenched in a limited time has the yield specified by (44) which completely neglects the subsequent delayed fluorescence resulting from the bulk recombination. Such a yield obeys the Stern-Volmer law (45) but with the geminate quenching constant $k_{q} \equiv \kappa_{g}$. The latter is concentration-independent unlike its analog in (46) accounting for the backward energy transfer during bulk encounters.

Hence, the luminescence quenched by reversible ionization after instantaneous excitation has the yield calculated from (45) and (56) [52]:

$$
\eta=\frac{1}{1+c \kappa_{g} \tau_{A}}
$$

where the geminate Stern-Volmer constant is $[43,52]$

$$
\kappa_{g}=\widetilde{R}^{*}(0)=\frac{\kappa_{i}}{1+\kappa_{i} / K\left(k_{D}+k_{c}\right)} .
$$

Here $\kappa_{i}$ is the Stern-Volmer constant for irreversible transfer (51) while

$$
K=\frac{k_{a}}{k_{b}}=\exp \left(-\frac{\Delta G_{i}}{T}\right)
$$

is an equilibrium constant for ionization, with free energy $\Delta G_{i}$ (here and further on the Boltzmann constant $k_{B}=1$ ). The kinetic reaction constants of the outer-sphere electron transfer

$$
k_{a}=k_{a}^{\prime} e^{-u_{a} / T}, \quad k_{b}=k_{b}^{\prime} e^{-u_{b} / T}
$$

obey the Arrhenius law with activation energies satisfying the free energy gap (FEG) law [9]:

$$
u_{a}=u_{b}+\Delta G_{i}=\frac{\left(\Delta G_{i}+\lambda_{c}\right)^{2}}{4 \lambda_{c}},
$$

where $\lambda_{c}$ is the contact reorganization energy of the polar media. The charge recombination constant also obeys the FEG law:

$$
k_{c}=k_{c}^{\prime} e^{-u_{c} / T} \quad \text { with } u_{c}=\frac{\left(\Delta G_{r}+\lambda_{c}\right)^{2}}{4 \lambda_{c}}
$$

but with different free energy $-\Delta G_{r}=\mathcal{E}+\Delta G_{i}$ where $\mathcal{E}$ is the excitation energy of $A^{*}$.

For highly exergonic charge separation $\left(-\Delta G_{i}>T\right)$ when $K \rightarrow \infty$ the reaction (55) becomes irreversible, that is, $\kappa_{g} \rightarrow \kappa_{i}$ which is the same for the irreversible transfer of either energy or electron. In the opposite limit ( $K \lesssim 1$ ), the transfer can also be irreversible provided the charge separation or their geminate recombination is fast: either $k_{D} \rightarrow \infty$ or $k_{c} \rightarrow \infty$. Otherwise, the distribution between neutral and charged reactants is equilibrated and the luminescence having the Stern-Volmer constant $\kappa_{g} \approx$ $K\left(k_{D}+k_{c}\right)$ disappears simultaneously with the ion pair that either separates or recombines. 
3.4. Stationary Luminescence. The pumping light intensity is $I=\left(N_{0} / N_{A}\right) \delta(t)$ in the case of pulse excitation but $I=$ $I_{0}=$ const when the fluorescence is an induced and studied stationary. In the former case, the quantum yield $\eta$ has to be calculated from formula (45) while in the latter case it is defined in another way $[53,54]$ :

$$
\eta=\frac{N_{A}^{s}}{I_{0} N_{A} \tau_{A}}=\frac{1}{1+c \kappa_{0} \tau_{A}},
$$

where $N_{A}^{s}$ is the stationary density of the excited states. If the fluorescence is quenched by reversible intramolecular transfer according to scheme (24), then both recipes were shown to give the same result [42]. A different situation arises when the quenching is performed by the intermolecular transfer whose charged products recombine in the bulk restoring the excitation according to scheme (30). In such a case, $N_{A}^{s}$ can be found from the set of corresponding IET equations (31) setting $\dot{N}_{A}^{*}=\dot{P}=0$. Substituting the stationary solution thus obtained into (64) we get the corresponding Stern-Volmer constant

$$
\kappa_{0}=\widetilde{R}^{*}(0)\left[1-\frac{\widetilde{R}^{\sharp}(0)}{\widetilde{R}^{\ddagger}(0)} \times \frac{\widetilde{R}^{\dagger}(0)}{\widetilde{R}^{*}(0)}\right]=\kappa_{g}[1-\chi \cdot \bar{\varphi}] .
$$

It is smaller than the geminate one $\left(\kappa_{g}=\widetilde{R}^{*}(0)\right)$ because not all the excitations are quenched forever at first encounter. Some of them are restored with an efficiency

$$
\chi=\frac{\widetilde{R}^{\ddagger}(0)}{\widetilde{R}^{\ddagger}(0)}
$$

in the subsequent bulk encounters of the free ions, which are separated with the yield

$$
\bar{\varphi}=\frac{\widetilde{R}(0)}{\widetilde{R}^{*}(0)} .
$$

All the components of (65) are well defined via the IET kernels.

Calculated in the contact approximation, they reduce expression (65) to the following one $[43,54]$ :

$$
\kappa_{0}=\frac{\kappa_{i}}{1+\kappa_{i} / K k_{c}} .
$$

The principle difference between this result and the geminate one, (59), is the absence of $k_{D}$ in the denominator. Diffusional ion pair separation cannot make the stationary energy quenching irreversible. The charge separation does not put an end to the reversible reaction, though interrupts it for a while. Only the irreversible recombination to the ground state of the neutral products proceeding with rate constant $k_{c}$ causes this to happen. If $k_{c} \rightarrow \infty$, the ionization is fully irreversible, that is, $\kappa_{0} \equiv \kappa_{i}$ Figure 12 .

On the contrary, at $k_{c}=0$ the quenching is reversible, that is, the fluorescence is not quenched at all $\left(\kappa_{0}=0\right)$. Almost the same is true when $K \gg \kappa_{i} / k_{c}$, where $K$ and $k_{c}$ were specified in (60) and (63). The quasireversible ionization is controlled by RIP recombination which proceeds with the rate constant

$$
\kappa_{0} \approx K k_{c}=k_{c}^{\prime} e^{-U / T} .
$$

The total activation energy of such a reaction,

$$
U=\Delta G_{i}+u_{c},
$$

becomes negative at highly exergonic transfer (when $\left|\Delta G_{i}\right|>$ $\left.u_{c}\right)$.

3.5. Association/Dissociation of the Exciplex. A different situation arises when the luminescence is interrupted by the reversible association of $A^{*}$ with impurity $(B)$ present in great excess, resulting in exciplex $\left(C^{*}\right)$ formation [55]:

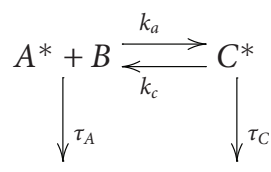

Here $\tau_{C}$ and $\tau_{A}$ are the decay times of bound and unbound excitation that may be either equal or different. The densities of the excited particles obey the set of IET equations:

$$
\begin{aligned}
\frac{d N_{A}^{*}}{d t}= & -c k_{a} \int_{0}^{t} \Sigma(t-\tau) N_{A}^{*}(\tau) d \tau \\
& +k_{c} \int_{0}^{t} \Sigma(t-\tau) N_{C}^{*}(\tau) d \tau-\frac{N_{A}^{*}}{\tau_{A}}, \\
\frac{d N_{C}^{*}}{d t}= & c k_{a} \int_{0}^{t} \Sigma(t-\tau) N_{A}^{*}(\tau) d \tau \\
& -k_{c} \int_{0}^{t} \Sigma(t-\tau) N_{C}^{*}(\tau) d \tau-\frac{N_{C}^{*}}{\tau_{C}} .
\end{aligned}
$$

The luminescence quantum yields of $A^{*}$ and $C^{*}$ are

$$
\eta_{A A}=\frac{1}{\tau_{A}} \int_{0}^{\infty} N_{A}^{*}(t) d t / N_{0}, \quad \eta_{C A}=1-\eta_{A A},
$$

provided that only $A$ is subjected to instantaneous light excitation: $N_{A}^{*}(0)=N_{0}, N_{C}^{*}(0)=0$.

The conventional Stern-Volmer law,

$$
\frac{1}{\eta_{A A}}=1+c \kappa \tau_{A} \quad \kappa=\frac{k_{a} \tilde{\Sigma}(0)}{1+k_{c} \tau_{C} \tilde{\Sigma}(0)},
$$

has the Stern-Volmer constant $\kappa$, which depends on the concentration via $\tilde{\Sigma}(0, c)$. Only its minimal value $\tilde{\Sigma}_{0}=\tilde{\Sigma}(0,0)$ calculated with IET from (73) and (72) is concentrationindependent:

$$
\begin{aligned}
& \kappa_{0}=\lim _{c \rightarrow 0} \kappa(c)=\frac{k_{a} \widetilde{\Sigma}_{0}}{1+k_{c} \tau_{C} \tilde{\Sigma}_{0}} \\
& \tilde{\Sigma}_{0}=\left[1+\frac{k_{a}}{k_{D}\left(1+\sqrt{\tau_{d} / \tau_{A}}\right)}\right]^{-1} .
\end{aligned}
$$

For the irreversible binding $\left(k_{c}=0\right)$ this $\kappa_{0}=k_{a} \widetilde{\Sigma}_{0}$ coincides with $\kappa_{i}$ given in (51).

Equations (72) as well as the general $\kappa$ definition given in (74) are common for all multiparticle theories. They differ only in $\tilde{\Sigma}(s)$ that were collected in [55, Tables 1 and 2] for the 


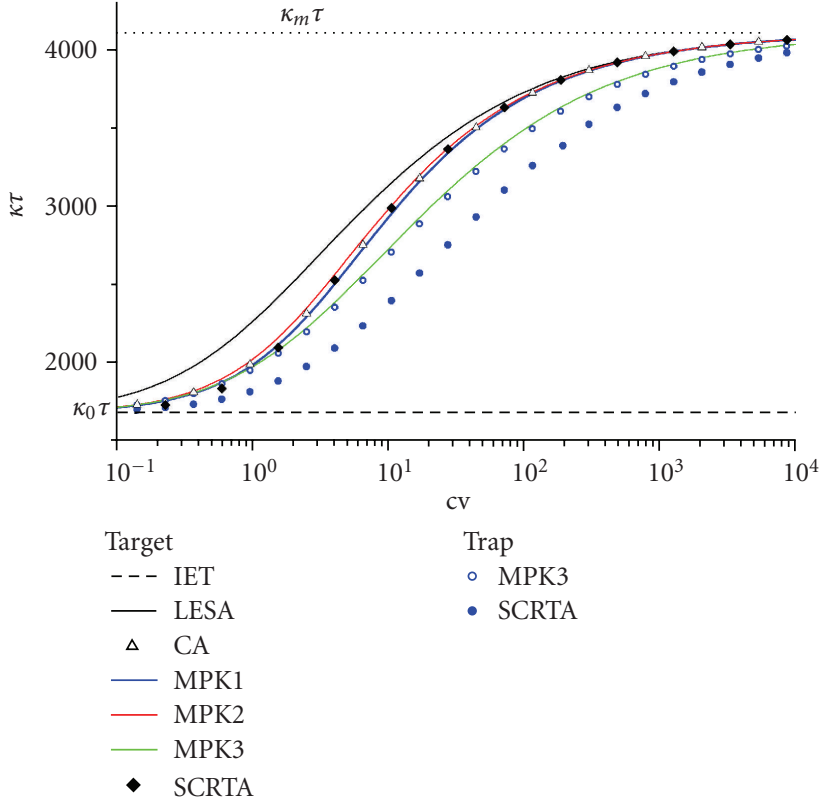

(a)

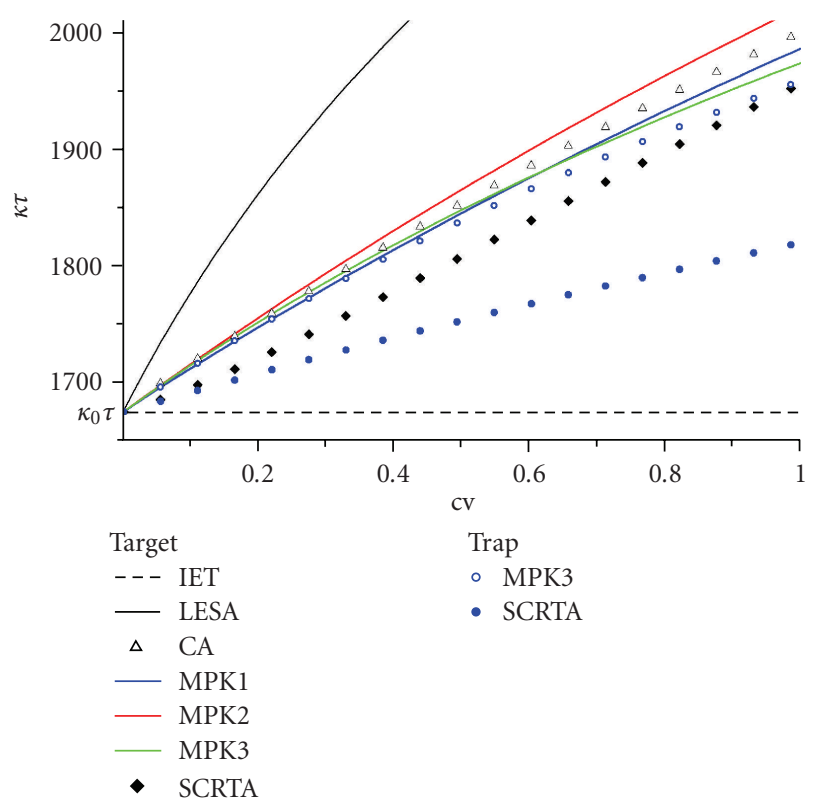

(b)

FIGURE 10: The Stern-Volmer constant of the reversible exciplex formation at $\tau_{A}=\tau_{C}=\tau$ as a function of the dimensionless concentration of $B$ 's. In a wide range of concentration (a) all curves increase from the minimal (IET) value $\kappa_{0}$ up to the maximal one, $\kappa_{m}$. At low concentrations (b) the difference between them is more pronounced [55].

target and trap problem (only $A$ is moving between immobile traps). All $\tilde{\Sigma}(0)$ monotonously increase with $c$ approaching 1 . Simultaneously the upper limit of the Stern-Volmer constant is achieved:

$$
\kappa_{m}=\frac{k_{a}}{1+k_{c} \tau_{C}} .
$$

Since only a few theories deal with $\tau_{A} \neq \tau_{C}$, in Figure 10, only the equal lifetimes case is examined. It is easy to see that all MPK theories and CA give similar results, unlike SCRTA and LESA which deviate from them into opposite sides. LESA was independently shown to give an inappropriate description of reversible transfer at equal times [42]. SCRTA in its turn strongly overestimates the difference between the target and trap problems as compared to MPK3 $\equiv$ MET. As to the concentration-independent IET result, it is always reproduced but only as $c \rightarrow 0$. At higher concentration it is better to replace IET with MET.

Quite recently the kinetics of energy quenching by exciplex formation and resulting free energy dependence of Stern-Volmer constant were thoroughly investigated with IET and compared with available experimental data [56].

3.6. Electroluminescence. The ions injected from electrodes recombine to either the ground or excited state of the neutral products. The latter can be detected by their luminescence and the quantum yield of such an electroluminescence is

$$
\phi_{\mathrm{ecl}}=\phi_{\mathrm{e}} \phi_{\mathrm{es}} .
$$

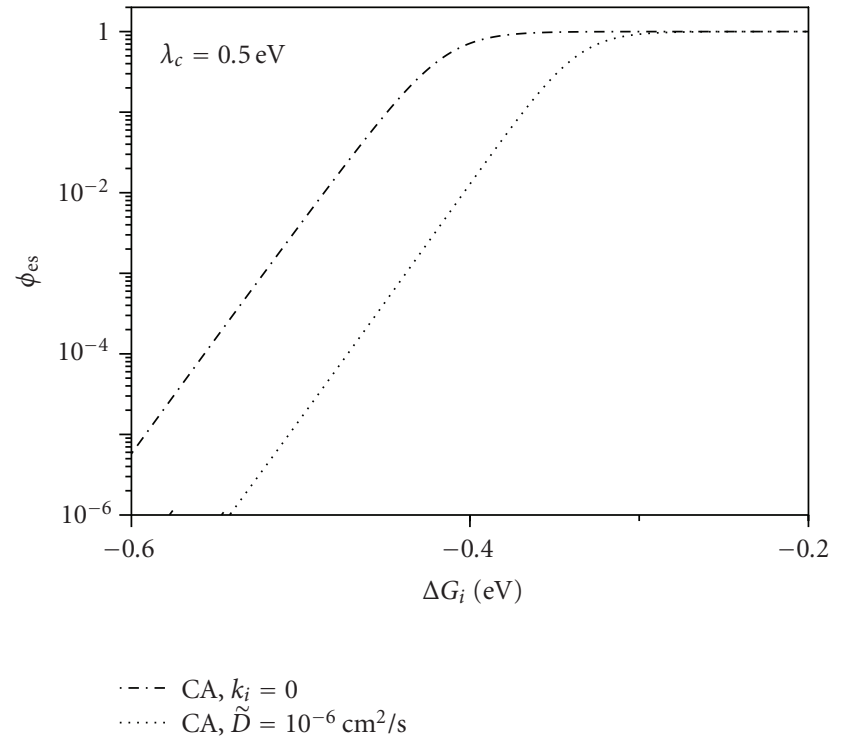

FIGURE 11: The quantum yield of the excited states, $\phi_{\text {es }}$, calculated in the contact approximation with (dashed line) and without (dasheddotted line), taking into account their ionization [43].

The quantum yield of excitations, $\phi_{\mathrm{es}}$, can be extracted from this relationship since the emission quantum yield from the excited state, $\phi_{\mathrm{e}}$, is usually known. In [57-59] the $\phi_{\mathrm{es}}$ dependence on the free energy of ionization, $\Delta G_{i}=-\Delta G_{b}$, 


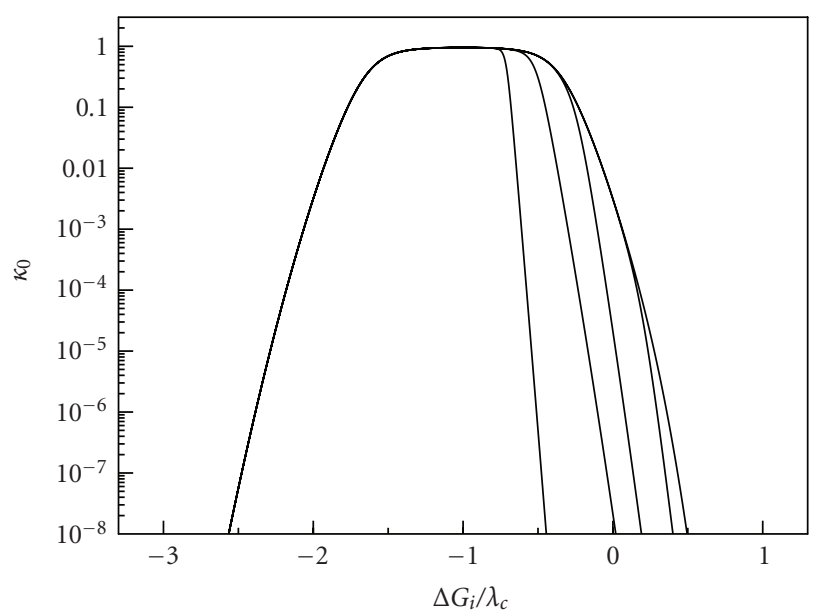

(a)

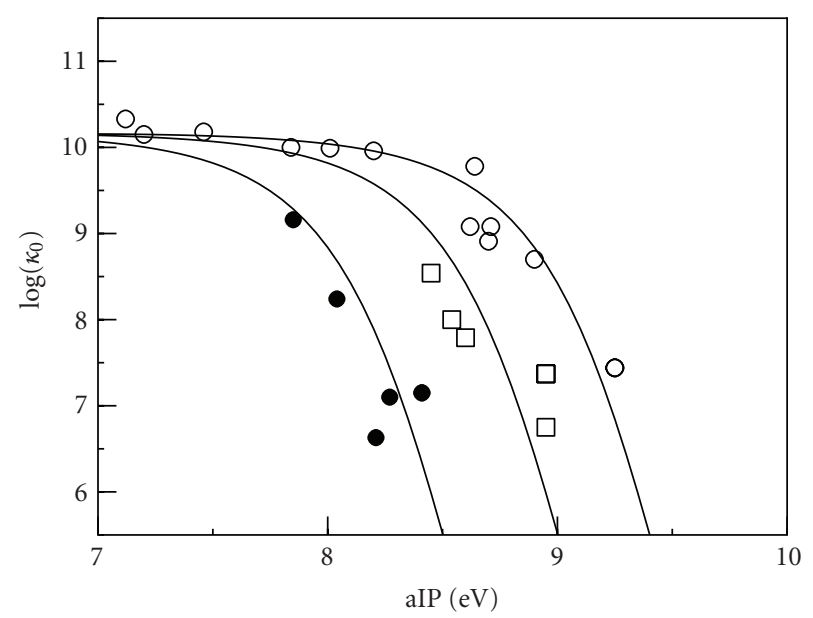

(b)

FIgURE 12: The Rehm-Weller plot for a few systems which differ by their triplet RIP recombination rate. (a) The theoretical curves for $k_{t}^{\prime} / k_{D}=0 ; 10^{-8} ; 10^{-5} ; 0.1 ; 20$ at $k_{c}^{\prime}=k_{b}^{\prime}=20 k_{D}$. (b) Interpolation through experimental points from [61, Figure 2].

was measured for a number of systems. To specify this dependence we have to calculate

$$
\phi_{\mathrm{es}}=\frac{\int_{0}^{\infty} N_{A}^{*}(t) d t}{P(0) \tau_{A}}
$$

borrowing $N_{A}^{*}(t)$ from the solution of (31) where we set $I_{0}=$ 0 and use the appropriate initial conditions created by the external injection of ions into solution:

$$
N_{A}^{*}(0)=0, \quad P(0)=P_{0} .
$$

Making the Laplace transformation of (31) we obtain from (78)

$$
\begin{aligned}
\phi_{\mathrm{es}} & =\frac{\widetilde{N}_{A}^{*}(0)}{P_{0} \tau_{A}} \\
& =\frac{1}{\widetilde{R}^{\ddagger}(0) / \widetilde{R}^{\sharp}(0)+c \tau_{A}\left[\widetilde{R}^{*}(0) \widetilde{R}^{\ddagger}(0) / \widetilde{R}^{\sharp}(0)-\widetilde{R}^{\dagger}(0)\right]} .
\end{aligned}
$$

Using the expressions for all the kernels obtained in contact approximation in [53] we get the contact analog of this equation [43]:

$$
\phi_{\mathrm{es}}=\frac{1}{1+\left(1+c \tau_{A} \kappa_{i}\right) k_{c} K / \kappa_{i}} .
$$

This expression reduces to a much simpler one provided the recombination into the excited state is irreversible as was assumed in [57-59]. If this is really the case, then $\kappa_{i}=k_{a}=0$ and $K / \kappa_{i}=K / k_{a}=1 / k_{b}$ so that (81) takes the form used in these works:

$$
\phi_{\mathrm{es}}=\frac{1}{1+k_{c} / k_{b}} .
$$

Using the contact estimates of the kinetic rate constants given in (61) and (63) we obtain the following final result:

$$
\phi_{\mathrm{es}}=\left[1+\frac{k_{c}^{\prime}}{k_{b}^{\prime}} \exp \left(-\frac{\mathcal{E}\left(\Delta G_{i}-\lambda_{c}+\mathcal{E} / 2\right)}{2 \lambda_{c} T}\right)\right]^{-1} .
$$

This is the stepwise function approaching unity when $\Delta G_{i}$ increases making the recombination to the excited state more favorable than to the ground one. Finally, the excitation becomes the unique reaction product since the recombination to the ground state is switched off.

Being calculated with (81), which accounts for the transfer reversibility (ionization of excited state), this function appears to be different from the simplest ones, (82) and (83), suited for irreversible recombination. Shown in Figure 11 these functions, although they are different, resemble the experimental results obtained in [57-59]. The correct accounting for the reaction reversibility is the main but not only advantage of IET, compared to DET and Markovian chemical kinetics. Taking into account the space dependence of the rates one should use (80) without contact simplifications and it was really employed in [43]. Moreover, in the next work the spin states of the free ions and radical ion pairs (RIPs) formed from them were also taken into account, as well as the spin conversion in the RIP and recombination to triplet products [60]. Even after that, the full correspondence with the experimental findings was not reached: the height of the true plateau remains lower than 1 for unknown reasons.

\section{Spin-Assisted Complex Reactions}

Until now we considered only the simplest reactions, which are sometimes termed as $A B C$ when dealing with exciplex formation (71) or $\mathrm{ABCD}$, when addressing either $A^{*} B A B^{*}$, or $A^{*} B A^{+} B^{-}$reactions (7), (49), and (50). It is rather easy to study the spinless reactants especially when transfer is contact and irreversible. However, the real chemistry deals with much more complex reactions than $\mathrm{ABC}, \mathrm{ABCD}$, and so on. They include the reactants with a few internal states and radical ions subjected to spin conversion and reverse recombination to their precursors and/or neutral products. 
Here we confine ourselves to spin-assisted contact reactions leaving noncontact effect for the next section.

Any realistic theory should discriminate between the singlet and triplet states of the radical-ion pair (RIP) and account for reversible transitions between them (Figure 13), either coherent (Hamiltonian) or incoherent with the model rate $k_{s}$. In general, the same is true for excited states of $A$ : singlet $\left({ }^{1} A^{*}\right)$ and triplet $\left({ }^{3} A^{*}\right)$, but the internal conversion is neglected here. If there are no triplet quenchers and the triplets are generated in a low concentration making their annihilation negligible, then the reaction scheme of reversible triplet production is the following one:

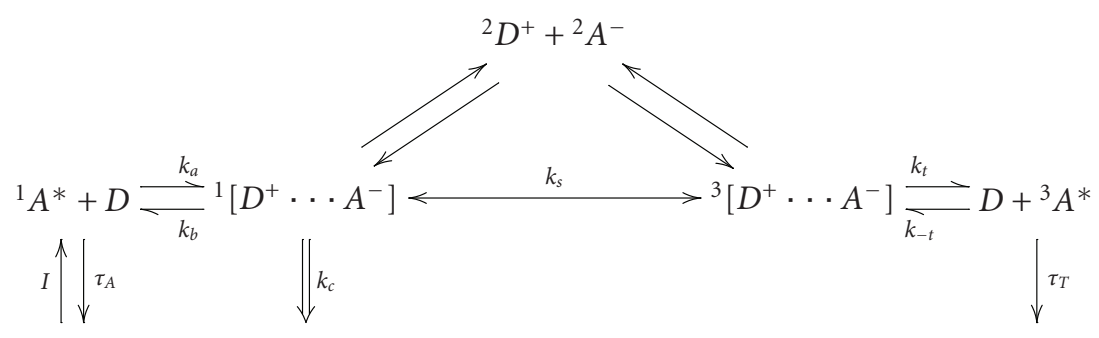

where $\tau_{A}$ and $\tau_{T}$ are the singlet and triplet life times. The forward and reverse electron transfer, to and from the excited triplet acceptor ${ }^{3} A^{*}$, has the rate constants $k_{t}$ and $k_{-t}$, respectively, which fit the detailed balance principle

$$
k_{-t}=k_{t} \exp \left(-\frac{\Delta G_{t}}{T}\right)
$$

where $\Delta G_{t}$ is the free energy of triplet ionization. There are two parallel ways of spin conversion: either within the geminate RIP or through the bulk where $1 / 4$ of the meetingfree ions associate into the singlet RIP and 3/4 into the triplet one.

The corresponding set of integral equations for the singlet and triplet populations, $N_{S}=\left[{ }^{1} A^{*}\right]$ and $N_{T}=\left[{ }^{3} A^{*}\right]$, and the concentration of charges, $P=\left[A^{-}\right]=\left[D^{+}\right]$, is the most complex one:

$$
\begin{aligned}
\dot{N}_{S}= & -\frac{N_{S}}{\tau_{A}}-c \int_{0}^{t} R^{*}(t-\tau) N_{S}(\tau) d \tau \\
& +\int_{0}^{t} R^{\sharp}(t-\tau) P^{2}(\tau) d \tau \\
& +c \int_{0}^{t} R^{\star}(t-\tau) N_{T}(\tau) d \tau,
\end{aligned}
$$

$$
\begin{aligned}
\dot{P}= & c \int_{0}^{t} R^{\dagger}(t-\tau) N_{S}(\tau) d \tau-\int_{0}^{t} R^{\ddagger}(t-\tau) P^{2}(\tau) d \tau \\
& +c \int_{0}^{t} R^{\diamond}(t-\tau) N_{T}(\tau) d \tau, \\
\dot{N}_{T}= & -\frac{N_{T}}{\tau_{T}}+c \int_{0}^{t} R^{\bullet}(t-\tau) N_{S}(\tau) d \tau+\int_{0}^{t} R^{\oplus}(t-\tau) P^{2}(\tau) d \tau \\
& -c \int_{0}^{t} R^{\star}(t-\tau) N_{T}(\tau) d \tau .
\end{aligned}
$$

There are 9 different kernels (memory functions), having separate IET definitions via different auxiliary functions obeying equations similar to (11). All of them were solved in the contact approximations. The kernels obtained were published in the appendix of [54] and later on in [62], where the general solution of the problem is given for both singlet and triplet luminescence.

4.1. Zero Spin Conversion. When the spin conversion in geminate pairs is rather slow, it can be neglected compared to the parallel track of triplet production; via bulk recombination of free ions into triplet RIPs:

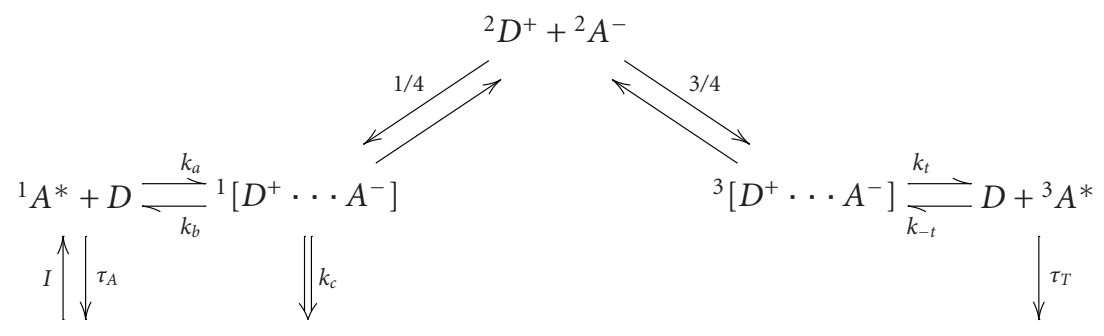




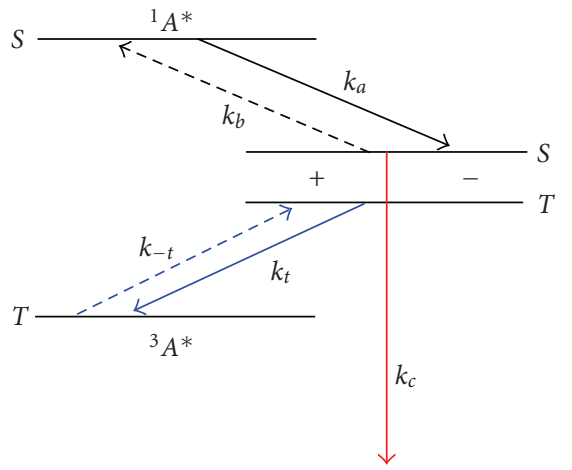

FIGURE 13: The energetic scheme of reversible ionization of singlet and triplet excitations, ${ }^{1} A^{*}$ and ${ }^{3} A^{*}$.

Under permanent illumination $I(t)=I_{0}=$ const, there are stationary populations of all species, $N_{S}^{s}, P_{s}, N_{T}^{s}$, which obey the set of equations following from (86):

$$
\begin{aligned}
\frac{N_{S}^{s}}{\tau_{A}}-I_{0} N_{A}= & -c \int_{0}^{t} R^{*}(t-\tau) N_{S}^{s}(\tau) d \tau \\
& +\int_{0}^{t} R^{\sharp}(t-\tau) P_{s}^{2}(\tau) d \tau \\
& +c \int_{0}^{t} R^{\star}(t-\tau) N_{T}^{s}(\tau) d \tau, \\
0= & c \int_{0}^{t} R^{\dagger}(t-\tau) N_{S}^{s}(\tau) d \tau \\
& -\int_{0}^{t} R^{\ddagger}(t-\tau) P_{s}^{2}(\tau) d \tau \\
& +c \int_{0}^{t} R^{\diamond}(t-\tau) N_{T}^{s}(\tau) d \tau, \\
\frac{N_{T}^{s}}{\tau_{T}}= & +c \int_{0}^{t} R^{\bullet}(t-\tau) N_{S}^{s}(\tau) d \tau \\
& +\int_{0}^{t} R^{\star}(t-\tau) P_{s}^{2}(\tau) d \tau \\
& -c \int_{0}^{t} R^{\star}(t-\tau) N_{T}^{s}(\tau) d \tau .
\end{aligned}
$$

After Laplace transformation, they become the algebraic equations for $N_{S}^{s}$ and other populations that can be easily found. Using the former in (64) instead of $N_{A}^{s}$, one can reproduce not only the general Stern-Volmer law but specify its constant (65) as well. The components of the latter are well defined through the Laplace transformations of the memory functions (kernels of (86)) [62].
At zero spin conversion $\left(k_{s}=0\right)$, they are

$$
\begin{aligned}
\kappa_{g} & =\widetilde{R}^{*}(0), \\
\chi & =\frac{\widetilde{R}^{\ddagger}(0)}{\widetilde{R}^{\ddagger}(0)} \cdot \frac{\widetilde{R}^{\star}(0) \widetilde{R}^{\ddagger}(0)}{\widetilde{R}^{\star}(0) \widetilde{R}^{\ddagger}(0)-\widetilde{R}^{\triangleleft}(0) \widetilde{R}^{\diamond}(0)}, \\
\bar{\varphi} & =\frac{\widetilde{R}^{\dagger}(0)}{\widetilde{R}^{*}(0)} .
\end{aligned}
$$

Calculating them in contact approximation, neglecting triplet decay, the following Stern-Volmer constant was obtained [62]:

$$
\begin{aligned}
\kappa_{0} & =\frac{\kappa_{i}}{1+\kappa_{i} / K k_{c}} \\
& = \begin{cases}\kappa_{i}=\frac{k_{a}}{1+k_{a} / k_{D}^{*}} & \text { as } k_{c} \longrightarrow \infty \text { (ionization control), } \\
k_{c} K & \text { as } k_{c} \longrightarrow 0 \text { (recombination control), }\end{cases}
\end{aligned}
$$

where $k_{D}^{*}=k_{D} /\left(1+\sqrt{\tau_{d} / \tau_{A}}\right)$. The reversible production of stable triplets does not affect this dependence identical to the spinless theory result (68).

As always one should discriminate between the ionization and recombination-controlled quenching. Under ionization control, the free energy dependence of $\ln \kappa_{0}$ reproduces that of $\ln \kappa_{i}$, which coincides with the bell-shaped FEG curve, $\ln k_{a}=-u_{a}\left(\Delta G_{i}\right) / T$, the top of which is cut by the diffusional plateau, $\ln \kappa_{i}=\ln k_{D}^{*}$. With increasing $\Delta G_{i}$, the irreversible singlet ionization becomes quasireversible and gives a way to recombination control, which turns down the free energy dependence of the Stern-Volmer constant and, the earlier, the slower is singlet RIP recombination (Figure 14). After the permanent illumination is suddenly switched off (as well as after $\delta$-pulse excitation), the luminescence goes out together with singlet excitations, $N_{S}(t)$. However, their population temporarily restores by recombination of ions into singlet: until the latter are reproduced by reverse electron transfer from the long-lived triplets. In this time domain, the reaction (87) proceeds from the right to left backing delayed luminescence.

Since the decay of singlets is the fastest process, the recombination of triplets proceeds quasistationary, so that, $N_{S}(t) \propto P^{2}(t) \propto N_{T}(t)$ and

$$
\begin{array}{r}
\dot{N}_{T}=-\frac{N_{T}}{\tau_{T}}-c k_{Q} N_{T}, \\
\text { where } k_{Q}=\widetilde{R}^{\star}(0)\left[1-\frac{\widetilde{R}^{\star}(0)}{\widetilde{R}^{\ddagger}(0)}\right] .
\end{array}
$$

In the contact approximation [62],

$$
k_{Q}=k_{-t} \frac{1+\kappa_{i} / k_{c} K}{1+\kappa_{i} / k_{c} K+\left(k_{-t}+4 k_{t}\right) / k_{D}+3 k_{t} / k_{c}} .
$$


The quasi-exponential quenching of triplets was actually detected recently in line with the luminescence quenching [63]. Some of the data obtained are shown in Figure 14. The circles and the lower curves obtained for quenching of Lumicrome (LC) by aliphatic amines relate to the kineticcontrolled transfer following classical FEG law which is the same for the Stern-Volmer and triplet quenching constants. The triangles and upper curves, obtained for LC quenched by aromatic donors relate to stronger electron transfer represented by diffusion by diffusional plateau which is higher for singlet quenching subjected to transient effect. Unfortunately, both plateaus are too long extending even into endothermic region. This paradox was solved only recently assuming that the quenching is due to exciplex formation [56].

4.2. Double-Channel Geminate Recombination/Separation. If the spin conversion is efficient, the geminate reaction followed pulse excitation produces a number of ions and triplets. The first experimental study of such a doublechannel reaction [64] raised a few questions about the accumulation kinetics and quantum yields of its products in solvents of different viscosities. The reaction scheme for the double-channel geminate reaction is easy to get from a general one, (84), by omitting all the bulk reactions:

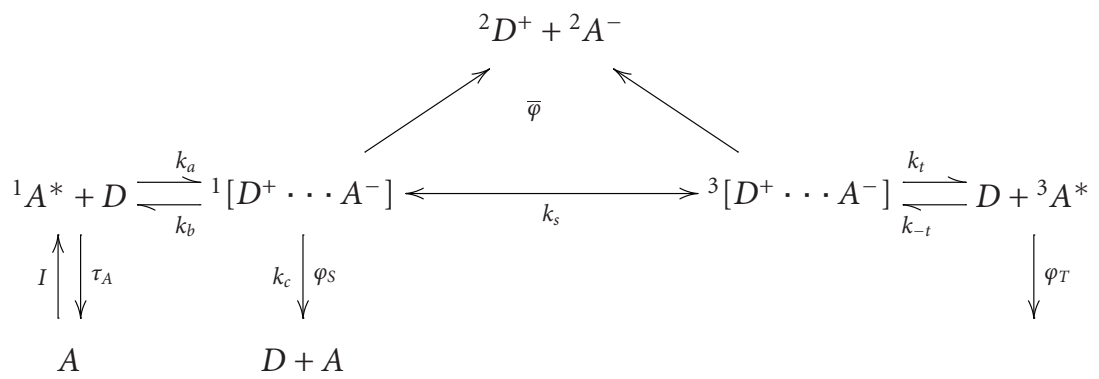

The yields of triplet products $\varphi_{T}$ and charge separation yield $\bar{\varphi}$ are two measurable quantities, while the yield of the ground state recombination products, $\varphi_{S}$, can be easily calculated from them: $\varphi_{S}=1-\varphi_{T}-\bar{\varphi}$ [64]. All yields are obtained from the limiting values of excitation, ions and triplet populations, which obey the following set of reduced equations:

$$
\begin{array}{cc}
\dot{N}_{S}=-\frac{N_{S}}{\tau_{A}}-c \int_{0}^{t} R^{*}(t-\tau) N_{S}(\tau) d \tau, & N_{S}(0)=N_{0}, \\
\dot{P}=c \int_{0}^{t} R^{\dagger}(t-\tau) N_{S}(\tau) d \tau, & P(0)=0, \\
\dot{N}_{T}=-\frac{N_{T}}{\tau_{T}}+c \int_{0}^{t} R^{\bullet}(t-\tau) N_{S}(\tau) d \tau, & N_{T}(0)=0 .
\end{array}
$$

In reality, $\tau_{T}$ is the longest time that may be set infinite, when the geminate reaction is studied. In such a case, one can easily find from the Laplace transformed equations (88) that

$$
\begin{gathered}
\tilde{N}_{S}(0)=\frac{N_{0} \tau_{A}}{1+c \tau_{A} \widetilde{R}^{*}(0)}, \\
P(\infty)=\lim _{s \rightarrow 0} s \widetilde{P}(s)=c \widetilde{R}^{\dagger}(0) \tilde{N}_{S}(0)=N_{0} \psi \bar{\varphi}, \\
N_{T}(\infty)=\lim _{s \rightarrow 0} s \widetilde{N}_{T}(s)=c \widetilde{R}^{\bullet} \widetilde{N}_{S}(0)=N_{0} \psi \varphi_{T},
\end{gathered}
$$

where the total yield of ions

$$
\psi=1-\eta=\frac{c \kappa_{g} \tau_{A}}{1+c \kappa_{g} \tau_{A}} .
$$

Here $\kappa_{g}=\widetilde{R}^{*}(0)$ is the same as in (89) while [62]

$$
\bar{\varphi}=\frac{\widetilde{R}^{\dagger}(0)}{\widetilde{R}^{*}(0)}, \quad \varphi_{T}=\frac{\widetilde{R}^{\bullet}(0)}{\widetilde{R}^{*}(0)} .
$$

If the spin conversion is negligible,

$$
\bar{\varphi}=\frac{1}{1+k_{c} / k_{D}}, \quad \varphi_{T}=0
$$

are exactly the same as in the spinless theory [9]. How these quantities are affected by spin-conversion, either coherent or incoherent, will be discussed later in the frame of the unified theory (Section 5.5).

Equations (88) describe the whole geminate reaction followed instantaneous creation of singlet excitation at $t=$ 0 . This reaction is composed from two sequential stages: accumulation and dissipation of ions. Another situation appears when the ions are created by a straightforward pulse excitation at the moment $t=0$. If the highly positioned singlet excitation is out of game $\left(k_{a}=k_{b}=0\right)$, the set of $(94)$ is reduced to the following one:

$$
\begin{gathered}
\dot{P}=c \int_{0}^{t} R^{\dagger}(t-\tau) N_{S}(\tau) d \tau, \quad P(0)=P_{0}, \\
\dot{N}_{T}=-\frac{N_{T}}{\tau_{T}}+c \int_{0}^{t} R^{\bullet}(t-\tau) N_{S}(\tau) d \tau, \quad N_{T}(0)=0 .
\end{gathered}
$$

The natural triplet decay is accompanied by their quenching by ionization and subsequent recombination of ions to either ground or triplet states. 


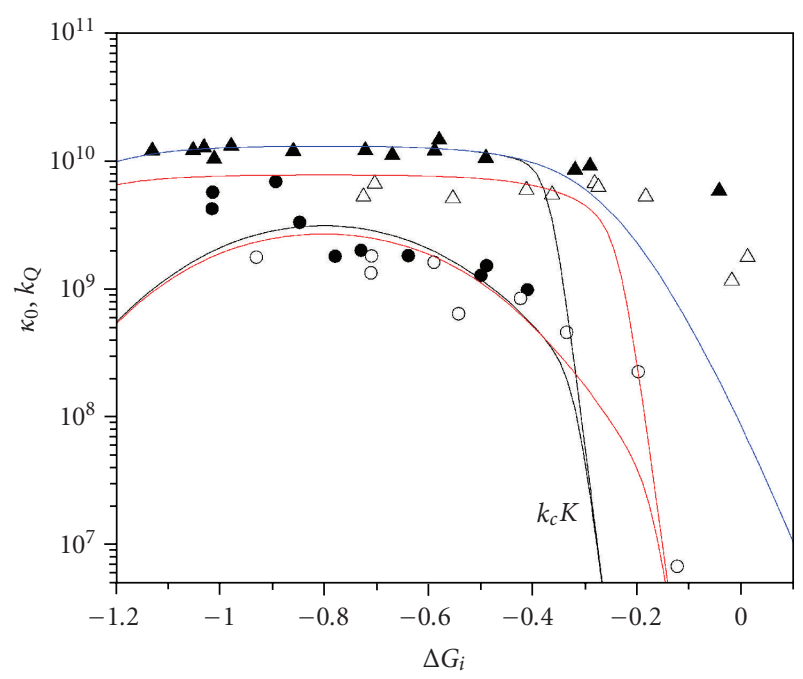

FIGURE 14: The experimental results from [63] for the Stern-Volmer constants $\kappa_{0}(\boldsymbol{\Lambda}, \bullet)$ and triplet quenching constants $k_{Q}(\Delta, \circ)$ fitted in [62]. The black lines are $\kappa_{0}$ and red ones are $k_{Q}$. The triangles and upper curves, calculated for strong electron transfer $\left(k_{a}=\right.$ $k_{t}=30 k_{D}$ ), are related to Lumicrome (LC) quenched by aromatic donors in methanol. The circles and the lower curves, obtained for weak kinetic controlled transfer $\left(k_{a}=k_{t}=0.5 k_{D}\right)$, belong to the LC quenched by aliphatic amines in the same solvent. The blue line shows the Stern-Volmer constant for irreversible ionization of singlet LC, $\kappa_{i}$, at the very same parameters.

4.3. Magnetic Field Effect in Double-Channel RIP Recombination. If besides that the ionization of triplet is also negligible $\left(k_{-t}=0\right)$, then the geminate reaction scheme (93) reduces to the simplest one:

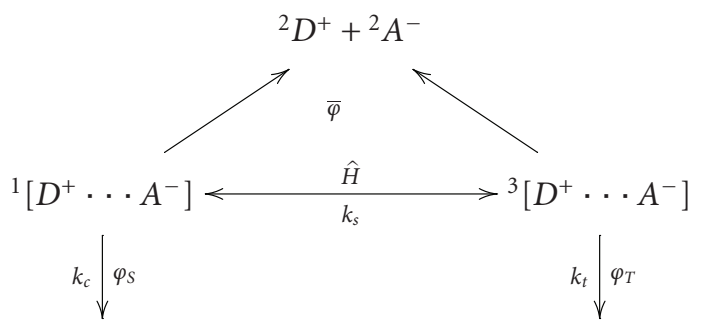

Since any electron transfer in this scheme is irreversible there is an alternative to IET how to find the solution of the problem by conventional methods of quantum chemistry [65]. In contact approximation, the total populations of charged and triplet products may be expressed via pair correlation functions of singlet and triplet RIPs, $m_{S}(r, t)$ and $m_{T}(r, t)$ :

$$
P(t)=\int\left[m_{S}(r, t)+m_{T}(r, t)\right] d^{3} r, \quad \dot{N}_{T}=k_{t} m_{T}(0, t) .
$$

The rate equations for these densities account for spin conversion in ion pair born at $r=r_{0}$ :

$$
\begin{gathered}
\dot{m}_{S}=-3 k_{s} m_{S}+k_{s} m_{T}+\widehat{L} m_{S}, \quad m_{S}(r, 0)=\frac{\delta\left(r-r_{0}\right)}{4 \pi r^{2}}, \\
\dot{m}_{T}=3 k_{s} m_{S}-k_{s} m_{T}+\widehat{L} m_{T}, \quad m_{T}(r, 0)=0,
\end{gathered}
$$

where

$$
\widehat{L}=\widetilde{D} \frac{1}{r^{2}} \frac{\partial}{\partial r} r^{2} e^{-U(r) / k_{B} T} \frac{\partial}{\partial r} e^{U(r) / k_{B} T}
$$

is an operator of the encounter diffusion (with coefficient $(\tilde{D}))$ in the interparticle potential $U(r)$. For an ion-radical pair this is the Coulomb interaction $U(r)=-r_{c} / r$, with Onsager radius $r_{c}=e^{2} / \epsilon k_{B} T$ (at temperature $T$ and dielectric constant $\epsilon$ ). This is the simplest but widely used elementary spin model (ESM) of incoherent spin-conversion in RIP with a single phenomenological parameter (rate) $k_{s}$ $[9,16]$.

The ion recombination into ground and triplet states can be taken into account via boundary conditions to (102):

$$
\begin{aligned}
& \left.4 \pi \sigma^{2} \tilde{D} e^{r_{c} / r} \frac{\partial}{\partial r} e^{-r_{c} / r} m_{S}\right|_{\sigma}=k_{c} m_{S}, \\
& \left.4 \pi \sigma^{2} \tilde{D} e^{r_{c} / r} \frac{\partial}{\partial r} e^{-r_{c} / r} m_{T}\right|_{\sigma}=k_{t} m_{T} .
\end{aligned}
$$

They represent the double-channel irreversible recombination at contact.

Equations (102) may be also written in operator form for two-component vector $\left(\begin{array}{c}m_{S} \\ m_{T}\end{array}\right)$ where $m_{T}=m_{-}+m_{0}+m_{+}$is actually a sum of three triplet components populations. For any particular mechanism of incoherent spin-conversion, the true rate equations should be written for either fourcomponent vector

$$
\left(\begin{array}{l}
m_{S} \\
m_{0} \\
m_{-} \\
m_{+}
\end{array}\right) \text {or density matrix } \rho=\left(\begin{array}{cccc}
m_{S} & * & * & * \\
* * & m_{0} & * & * \\
* * & * & m_{-} & * \\
* * & * & * & m_{+}
\end{array}\right)
$$

with account of real transitions between the components peculiar to the chosen mechanism. For instance, the stochastic Liouville equation for the density matrix of Ruthenium complex was specified in $[66,67]$ for the $\Delta g$-mechanism of spin conversion governed by the spin Hamiltonian

$$
\mathbf{H}=\frac{1}{2}\left(\begin{array}{cccc}
\omega_{+} & 0 & 0 & 0 \\
0 & -\omega_{+} & 0 & 0 \\
0 & 0 & 0 & \omega_{-} \\
0 & 0 & \omega_{-} & 0
\end{array}\right)
$$

Here

$$
\omega_{ \pm}=\frac{\left(g_{+} \pm g_{-}\right) \beta}{\hbar} H,
$$

$\beta$ is the Bohr magneton, $g_{+}$and $g_{-}$are $g$-factors of positive and negative ions and $H$ is the magnetic field. The relaxation superoperator that has the rank $16 \times 16$ in the Liouville space was specified in $[66,67]$ as well.

For moderate magnetic field and relatively fast transversal relaxation rate

$$
\omega_{-}=\frac{\Delta g \beta H}{\hbar} \ll \frac{1}{T_{2}},
$$


the quasistationary solution for all off-diagonal elements is allowed [9, Section VIII A]. It reduces the coherent description of density matrix evolution to the incoherent one which substitutes the phenomenological ESM set (102) by the more appropriate one $[9,68,69]$ :

$$
\begin{aligned}
\dot{m}_{S}= & \left(k_{0}+\frac{1}{T_{2}}-\frac{1}{2 T_{1}}\right) m_{0}-\left(k_{0}+\frac{1}{T_{2}}+\frac{1}{2 T_{1}}\right) m_{S} \\
& +\frac{m_{+}+m_{-}}{2 T_{1}}+\widehat{L} m_{S}, \\
\dot{m}_{0}= & \left(k_{0}+\frac{1}{T_{2}}-\frac{1}{2 T_{1}}\right) m_{S}-\left(k_{0}+\frac{1}{T_{2}}+\frac{1}{2 T_{1}}\right) m_{0} \\
& +\frac{m_{+}+m_{-}}{2 T_{1}}+\widehat{L} m_{0}, \\
\dot{m}_{+}= & \frac{m_{S}+m_{0}}{2 T_{1}}-\frac{m_{+}}{T_{1}}+\widehat{L} m_{+}, \\
\dot{m}_{-}= & \frac{m_{S}+m_{0}}{2 T_{1}}-\frac{m_{-}}{T_{1}}+\widehat{L} m_{-},
\end{aligned}
$$

where the ab-initio-derived rate of spin-conversion is

$$
k_{0}=\omega_{-}^{2} T_{2} .
$$

The rest depends on the relationship between the transversal $\left(T_{2}\right)$ and longitudinal $\left(T_{1}\right)$ relaxation times. At $T_{1}=\infty$ the general set (109) reduces to only two equations for $S$ and $T_{0}$ components [68] which are converting with the rate

$$
k_{s}=k_{0}+\frac{1}{T_{2}} .
$$

On the other hand, for $T_{2}=T_{1}=T_{0}$ the ESM equations (102) are approved but with another conversion rate [68]:

$$
k_{s}=\frac{1}{2 T_{0}}+\frac{1}{3} k_{0} .
$$

It was proven by the exact analytic solution of the general set (109) for a contact born pair $\left(r_{0}=\sigma\right)$. The comparison of exact double-channel solution from [70] with that of ESM obtained in [64] showed that they are exactly the same only at zero magnetic field $\left(k_{0}=0\right)$ when conversion is carried on by the transversal relaxation but differ a bit even in the lowest order approximation with respect to

$$
k_{0} T_{0}=\left(\omega_{-} T_{0}\right)^{2} \ll 1,
$$

which is quadratic in magnetic field $\left(\omega_{-} \propto H\right)$.

To remove any limitations, on magnetic field or other strength of spin conversion, one should give up the incoherent (rate) description of such a process and dill with the original operator equations in Liouville space. This was first done for a very special case of "spin-independent recombination" when RIP recombination through both channels is the same: $k_{c}=k_{t}[71]$. The authors considered simultaneous action of $\Delta g$ and HFI mechanisms of spin-conversion. The former was considered separately later on setting $k_{t}=0$

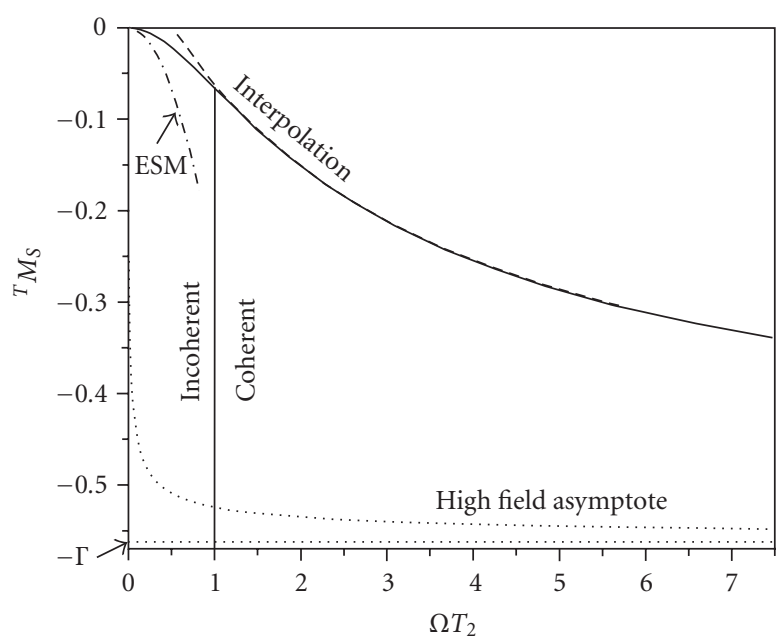

Figure 15: The field dependence of the MFE at contact start in the exact theory (solid line) and in the elementary spin model (dasheddotted parabolic line). The vertical line separates the low field (incoherent) $\Omega$-dependence from the high field MFE, originating from the coherent spin conversion. The latter is well interpolated by the empirical formula shown as the dashed curve approaching the exact result from above. The highest field asymptotic behavior and its limit, $-\Gamma$, are shown by the dotted lines below. The rate of contact recombination, $z_{s}$, and other parameters are the same as in the previous figure while $D=10^{-6} \mathrm{~cm}^{2} / \mathrm{s}$. $\left(\omega_{-}=2 \Omega\right.$. $)$

that is leaving only single (singlet) recombination channel [69]. The double recombination via $\Delta g$-mechanism, at any $k_{c}$ and $k_{t}$, was considered rather recently [72, 73]. The exact analytical solution of this problem essentially corrects the results obtained earlier with incoherent approximation even within the limits of their validity. This indicates the main weakness of the rate theory first reducing the coherent spin conversion to incoherent and only then accounting for the encounter diffusion and recombination of radicals. The exact theory does the opposite: first solves the problem by simultaneously taking into account the relative motion, recombination and conversion and only then turn to a limit where the incoherent approximation is assumed to be right. Unfortunately, this is true for only zero field case while the magnetic field effect is reproduced by ESM only qualitatively (Figure 15) and only for law fields limited by inequality (113).

4.4. Viscosity Dependence of the Double-Channel Recombination Yields. The coherent HFI-induced spin conversion was studied even earlier for a simplified model of a single-proton spin $\hat{\mathbf{I}}$ interacting with the electron spin $\widehat{\mathbf{S}}_{1}$ of that ion-radical where the proton is located [74]. The corresponding term in spin Hamiltonian of RIP, $A \widehat{\mathbf{I S}}_{1}$, contains the HFI constant $A$ which is the fitting parameter of the system instead of $k_{s}$. Accounting for this interaction and electron spin exchange at the closest distance, this problem was also solved analytically for zero field, and viscosity dependence of the yields (97) was specified at different starting distance $r_{0}$. The diffusional dependence of $\varphi_{T}$ compared with its ESM analogs was shown 


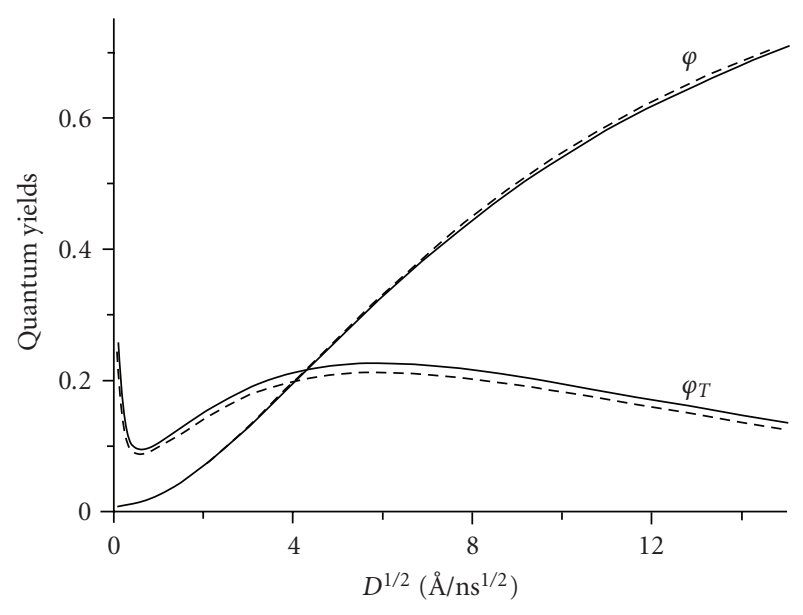

Figure 16: The charge separation, $\bar{\varphi}$, and triplet quantum yield, $\varphi_{T}$, as functions of diffusion started from $r_{0}=7.55 \AA>\sigma(\sigma=7.5 \AA)$. At zero magnetic field the recombination rates through singlet and triplet channels are $k_{c}=4800 \AA^{3} / \mathrm{ns}$ and $k_{t}=50000 \AA^{3} / \mathrm{ns}$ while the phenomenological rate of spin conversion is $k_{s}=0.15 \mathrm{~ns}^{-1}$ versus HFI constant $A=4.8 \mathrm{~ns}^{-1}$. The solid lines result from the coherent theory of HFI-induced spin conversion, while the dashed lines are obtained with the model (incoherent) theory at $k_{s}=A / 32$.

to be very similar (see [74, Figure 1]). The closest similarity was reached after special investigation of this problem in [75] accounting for Coulomb interaction between the ions. The triplet yield dependence on Onsager radius as well as on encounter diffusion was shown to be practically the same provided the phenomenological spin conversion rate $k_{s}$ relates to HFI constant $A$ as follows:

$$
k_{s}=\frac{A}{32} .
$$

The diffusional dependencies of charge separation and triplet production yields calculated with a model (incoherent) theory and its coherent (HFI) analog are shown also in Figure 16. Their coincidence under condition (114) is almost perfect, but the advantage of coherent theory is the possibility to get $A$ independently, from the ESR spectroscopy, and thus to verify the fitting objectively if $A$ is the same.

\section{Remote Transfer}

No matter how perfect is the fitting of the contact theories to the $\mathrm{BD}$ simulations, these theories are not good enough to treat the real experimental data. The main advantage of the encounter theories compared to their contact alternatives is their accounting for the true space dependence of the transfer rates. In the case of electron transfer, the most common is the usage of the so-called Marcus transfer rate, which is actually the perturbation theory estimate of the transition rate between the parabolic terms [76-78]:

$$
W(r)=U \exp \left(-\frac{(\Delta G+\lambda)^{2}}{4 \lambda T}\right)
$$

where $\Delta G$ is constant in highly polar liquids while

$$
\begin{gathered}
U(r)=\frac{V_{0}^{2}}{\hbar} \exp \left(-\frac{2(r-\sigma)}{L}\right) \frac{\sqrt{\pi}}{\sqrt{\lambda T}}, \\
\lambda(r)=\lambda_{0}\left(2-\frac{\sigma}{r}\right) .
\end{gathered}
$$

The contact reorganization energy,

$$
\lambda_{0}=\frac{e^{2}}{\sigma}\left(\frac{1}{n^{2}}-\frac{1}{\epsilon}\right)
$$

depends on the static dielectric constant $\epsilon$ and the refraction index $n$ which are usually known but specific for any particular solvent.

The electron coupling $V_{0}$ and tunneling length $L$ are the main fitting parameters of noncontact theory, instead of a single contact constant $\int W(r) d^{3} r$. However, the highly exergonic transfer is accompanied by vibrational excitations of the final state, so that such a multichannel transfer has the rate

$$
W(r)=U(r) \sum_{0}^{\infty} e^{-S} \frac{S^{n}}{n !} \exp \left[-\frac{(\Delta G+\lambda+\hbar \omega n)^{2}}{4 \lambda T}\right],
$$

where $S=\lambda_{q} / \hbar \omega$, while $\omega$ is the frequency and $\lambda_{q}$ is the reorganization energy of a single-assisted quantum mode.

One should discriminate between the transfer in the normal Marcus region $(-\Delta G<\lambda)$ and in the inverted one $(-\Delta G>\lambda)$. In the former the rate decreases monotonously and quasiexponentially [79] with distance (Figure 17), while in the latter it is bell-shaped and remote but shifts backward to contact if the transfer becomes multichannel (Figure 18). Two approximate models are used to simplify these alternative dependencies:

$$
W(r) \approx \begin{cases}W_{c} \exp \left(-\frac{2(r-\sigma)}{l}\right) & \text { normal region, } \\ \frac{W_{0}}{c h^{2}}\left(\frac{r-R}{\Delta}\right) & \text { inverted region. }\end{cases}
$$

The exponential model contains the effective tunneling length $l$ whose analog is $\Delta$ in the bell-shaped $W(r)$ proposed in [80]. Keeping it the same, the bell-shaped curve is sometimes substituted by the rectangular one $[16,81,82]$.

5.1. Transient Quenching Kinetics. The nonexponential kinetics of irreversible energy quenching is represented in DET by the solution of (3):

$$
N_{A}^{*}=N_{0} e^{-c \int_{0}^{t} k_{A}\left(t^{\prime}\right) d t^{\prime}} .
$$

The non-Markovian rate constant $k_{A}(t)$ given in the contact approximation by (2) diverges at the very beginning: $k_{A}(0)=$ $\infty$. The same quantity in the encounter theory, (5), tends to kinetic rate constant at $t \rightarrow 0$ :

$$
k_{A}(0)=\int W_{A}(r) d^{3} r=k_{a}, \quad \text { so that } \ln \frac{N_{A}^{*}}{N_{0}}=-c k_{a} t .
$$




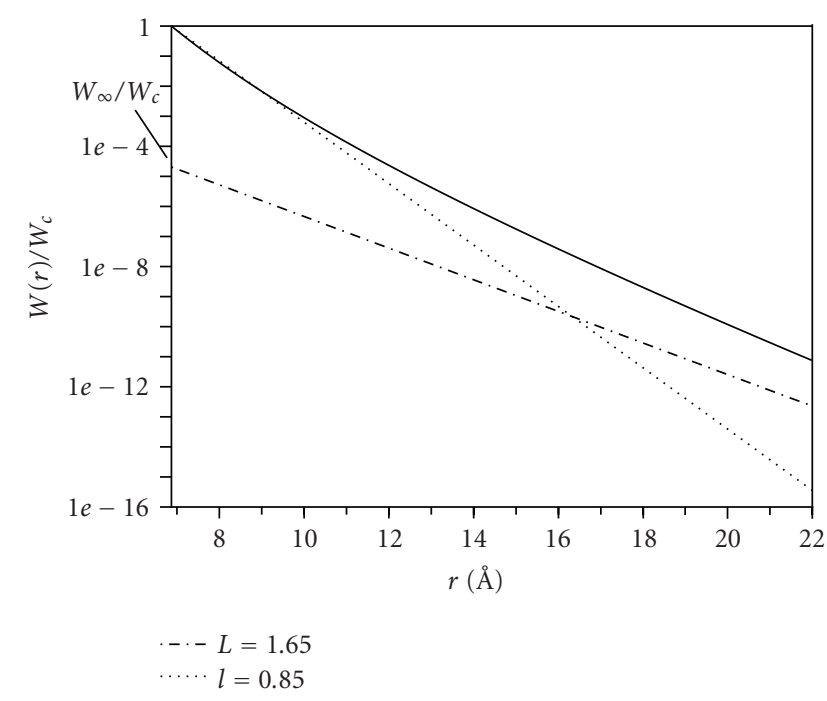

FIgURE 17: The distance dependence of the Marcus transfer rate (115) in the normal region $(\Delta G=-0.59 \mathrm{eV})$ with $L=1.65 \AA$ and $\lambda_{0}=1.3 \mathrm{eV}$ (thick line) in comparison to its exponential approximations for short (dotted line) and long (dashed-dotted line) distances. From [79].

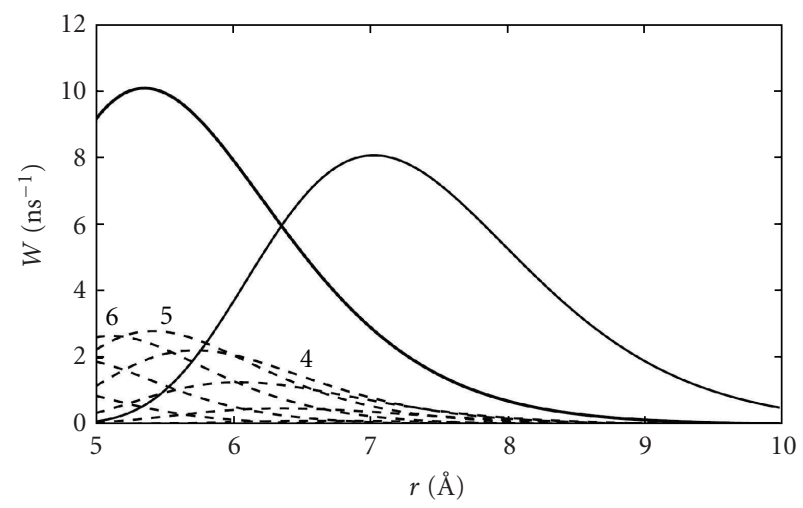

FIGURE 18: The rates of ionization in the inverted Marcus region $\left(-\Delta G=2 \lambda_{0}\right)$ in a polar solvent $\left(r_{c}=7 \AA, \lambda_{0}=1.4 \mathrm{ev}\right.$, $\sigma=5 \AA, T=300 \mathrm{~K}, \hbar \omega / T=6)$. The single channel reaction $(S=0)$ is exhibited by the right curve while the multichannel reaction $(S=2)$ is represented by the left one. The latter is decomposed in contributions related to different channels (dashed lines) nominated by the numbers of vibronic states $n$.

Only in the opposite limit (at long times) does the general asymptotic expression for $k_{A}(t)$ have the same shape as (2), except that the effective reaction radius $R_{Q}$ is substituted for $\sigma$. Integrating this expression in (120), the following long time asymptote for quenching kinetics is obtained:

$$
\begin{array}{r}
\ln \frac{N_{A}^{*}}{N_{0}}=-c\left[4 \pi R_{Q} D t+8 R_{Q}^{2} \sqrt{\pi D t}\right] \\
\text { as } t \longrightarrow \infty .
\end{array}
$$

The first term in the rhs of this equation represents the final exponential energy quenching with stationary rate constant $k=4 \pi R_{Q} D$, while the second one describes the transient

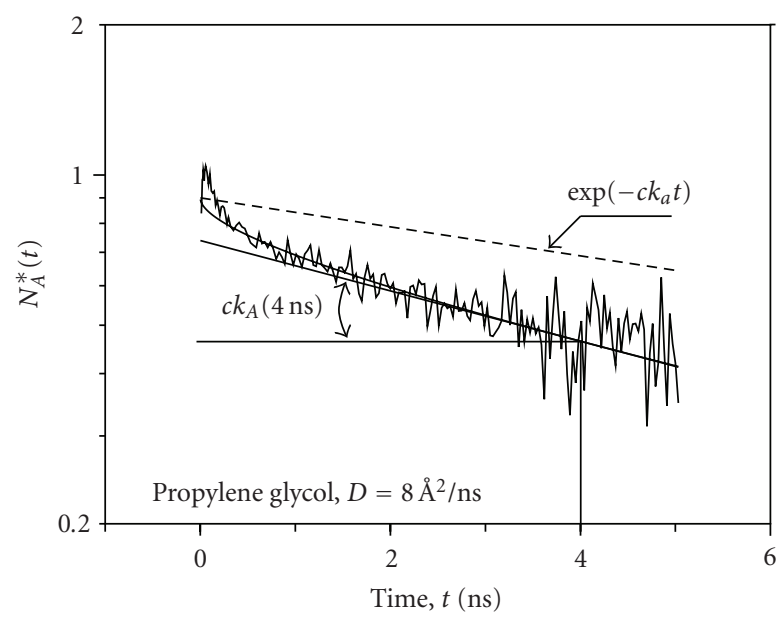

Figure 19: The fit of the non-stationary electron transfer kinetics (thick curve) to the experimental data obtained in [83]. The thin line represents the tangent to the kinetic curve at the largest time available $(t=4 \mathrm{~ns})$. Its slope is the time dependent rate of ionization, $c k_{A}(t)$, which differs essentially from that of the purely exponential decay, with the stationary rate constant $k_{a}=4 \pi R_{Q} D$ (dashed straight line). (From [79]).

effect: the preceding nonexponential development, though determined by the same $R_{Q}$.

The first attempt to extract $R_{Q}$ from the experimentally studied quenching of pheophytin $a$ by toluquinone [8] was not flawless. Since the short times were hardly available for the techniques of those times, the detected long time quenching was thought to be exponential. Thus obtained the pseudostationary rate constant $k$ was greatly overestimated, as well as $R_{Q}=k / 4 \pi D$. Its diffusional dependence was specified using 40 solvents of different viscosities but the tunneling length extracted from fitting DET to the thus obtained $R_{Q}(D)$ dependence was also significantly overestimated.

Only 10 years later, the quenching of Rhodamine 3B by N.N-dimethyleaniline was first recognized as a nonstationary one and $R_{Q}$ was obtained from fitting the asymptote (122) to better data and in a wider time interval [79]. As can be seen from Figure 19, the current $k_{A}(t)$ is essentially larger in its asymptotic value $k_{a}$, even at the longest attainable time $t=4$ ns. Hence, the whole quenching kinetics available in this study is nonstationary and should be fitted with an appropriate asymptotic expression, (122). The single quenching parameter $R_{Q}$ was obtained from this fit, employing 7 solvents of different viscosity and such obtained $R_{Q}(D)$ dependence, compared with the theoretical predictions of the contact theory and DET.

The results of this comparison are shown in Figure 20(a). At fast diffusion (under kinetic control), there is an agreement with all theories but at slow diffusion the experimental points lay far above the predictions of Collins-Kimball model following from (4):

$$
R_{Q}=\sigma \frac{k_{a}}{k_{a}+4 \pi \sigma D} .
$$




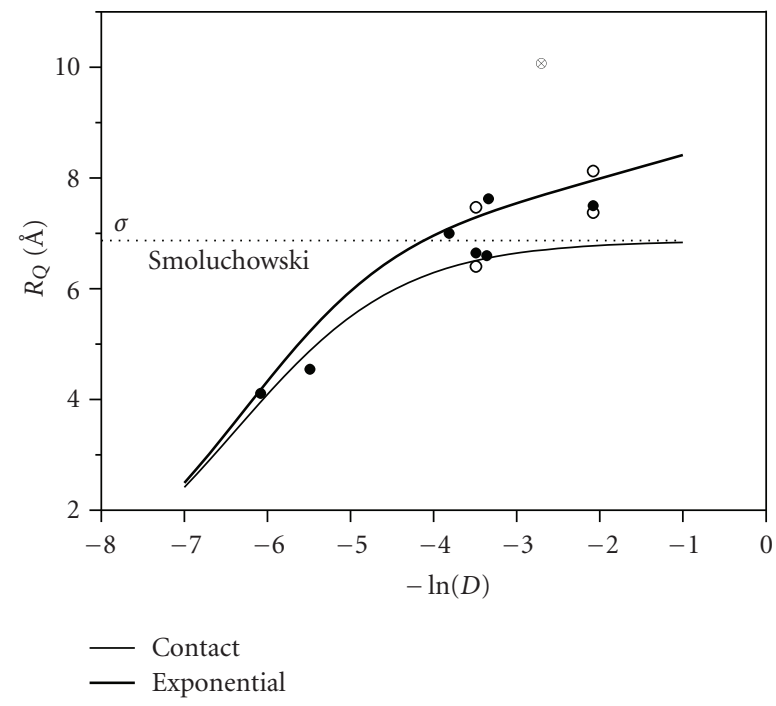

(a)

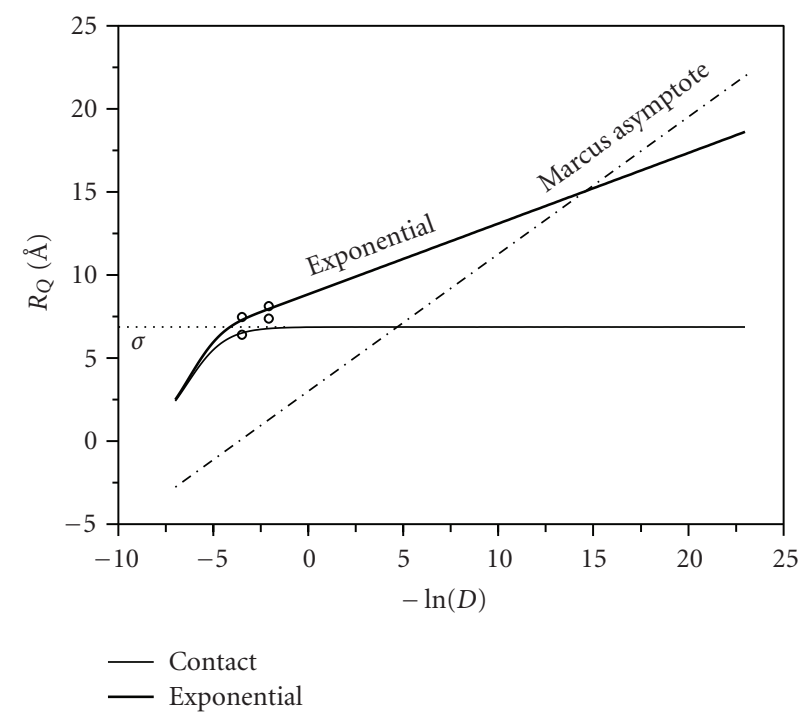

(b)

Figure 20: The dependence on diffusion of the effective electron transfer radius, $R_{Q}$. (a) The experimental data, indicated by circles, is approximated by a thin line representing the contact Smoluchowski-Collins-Kimball approach and by thick line depicting the same dependence, but for the exponential transfer rate from (119) with $l=0.85 \AA$ and $W_{c}=180 \mathrm{~ns}^{-1}$. (b) The same but in a wider diffusion diapason. The dashed-dotted line at the bottom represents the high viscosity asymptote of the same dependence but for the Marcus transfer rate. (From [79]).

Much better fitting is achieved with the exponential rate model (119), using the exact analytic expression for $R_{Q}(D)$ available in DET [7]. At fast and slow diffusion this expression has the following simple asymptotes:

$$
R_{Q}= \begin{cases}\sigma \frac{k_{a}}{4 \pi \sigma D} & \text { at } R_{Q}<\sigma \text { (kinetic control), } \\ \sigma+\frac{l}{2} \ln \left(\frac{\gamma^{2} W_{c} l^{2}}{4 D}\right) & \text { at } R_{Q}>\sigma \text { (diffusional control), }\end{cases}
$$

where $\gamma=\exp (C)$ and $C$ is the Euler constant. However, the real points are in between these limits and are well fitted with only the general solution for exponential $W_{A}(r)$ (Figure 20). The latter is also just a short distance approximation for the true Marcus rate. At larger distances the latter is also exponential but with true $L$ (Figure 17). The substitution of $L \approx 2 l$ for $l$ in (124) makes the slope of $R_{Q}(\ln D)$ at the highest viscosities almost twice as large but this region was not attainable with the solvents studied (Figure 20(b)). However, even at moderate viscosities the excitations never reach contact if ionization is under diffusional control. They are quenched farther apart, at $R_{Q}>\sigma$, and this is a noncontact reaction accessible for only DET or IET of the remote transfer.

By now, the best fitting of the transient effect and the whole ionization kinetics was made in [48] where the subpicosecond kinetics of the irreversible double-channel electron transfer (50) was studied. This is the highly exergonic quenching of Perylene (Per) by tetracyanoethylene (TCNE) producing the ion pairs in their ground and excited states. The pulse-induced quenching kinetics $N_{A}^{*}(t)$ was accurately studied in three different time intervals. The shortest and the longest ones were fitted with asymptotes (121) and (122), respectively, for specifying $k_{a}$ and $R_{Q}$ while from the middle one the relative strength of the parallel channels was inferred. The whole kinetics $N_{A}^{*}(t)$ appeared to be fitted perfectly, within the accuracy of experimental data (Figure 21).

5.2. Stern-Volmer Constant. The concentration dependence of the quantum yields or the Stern-Volmer constant is actually the central problem under investigation with numerous contact theories. Yet the noncontact DET corrects essentially even this dependence obtained with contact DET or its Smoluchowski-like analogs. To make this point clear a straightforward fitting of the remote transfer theory to the data presented by Stevens and Biver III [84] was undertaken in [47].

The highly exergonic fluorescence quenching of 9,10dicyanoanthracene (DCNA) by $\mathrm{N}, \mathrm{N}, \mathrm{N}^{\prime}, \mathrm{N}^{\prime}$-tetramethyl- $p$ phenylenediamine (TMPD) was studied in [84] in the broad range of TMPD concentrations, at three different temperatures $\left(T=0^{\circ}, 22^{\circ}, 50^{\circ} \mathrm{C}\right)$ in acetonitrile solutions. Neither a contact nor exponential approximation of the ionization rate $W_{I}(r)$ is good for the real fitting of this data. Instead, one should use the true Marcus rate of electron transfer accounting for the energy balance and the properties of the solution. The results of the best fit are shown in Figure 22. The theoretical constants (thick curves), depending on the dimensionless concentration $c v=\xi$, are in good agreement with the experimental points in [84]. The agreement could even be better if a number of additional factors were taken into account: the liquid structure near the contact, the spacial dependence of the 


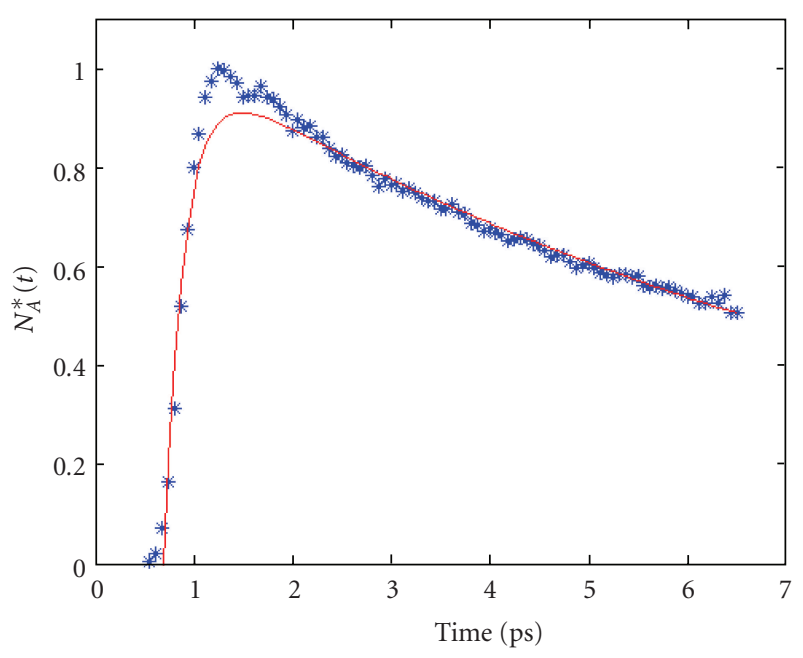

(a)

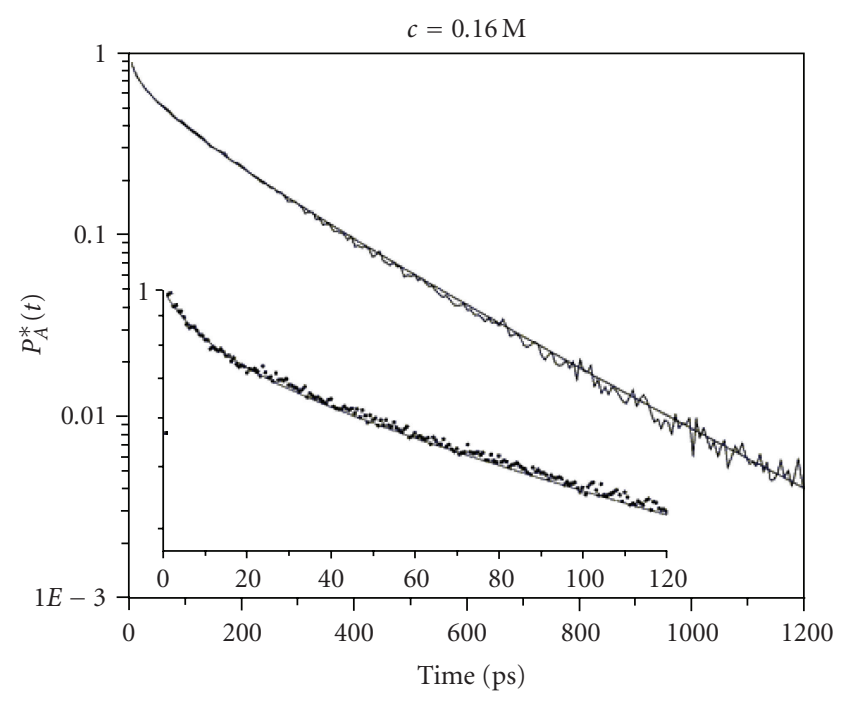

(b)

Figure 21: Fitting the double channel ionization kinetics $N_{A}^{*}=P_{A}^{*} \exp \left(-t / \tau_{A}\right)$ at the shortest time (above) as well as at the longest and medium ones (below). From [48].

diffusional coefficients [85], and the chemical anisotropy which is sometimes essential $[86,87]$. Neglecting all these factors, only two fitting parameters were used, the tunneling length $L$ and the electron coupling element $V_{0}$, which are found to be [47]: $L=1.6 \AA, V_{0}=4.07 \cdot 10^{-2} \mathrm{eV}$. However, even the simplest theory of the remote transfer fits the actual experimental data much better than its contact analog (thin curves in Figure 22).

It is also illustrated in Figure 23 borrowed from [48]. The fluorescence quantum yield obtained in pulse experiment was compared in this work [48] with that measured stationary (Figure 23(a)). Generally, they are in conformity with each other as well as with that calculated theoretically. The latter was obtained by integrating in (44) the best fitted $N_{A}^{*}(t)$ from Figure 21 . This integration can be restricted by the duration of the pulse experiment or extended to infinity taking into account the delayed fluorescence (contributed into the stationary luminescence). The Stern-Volmer constants subtracted from all the data are shown in Figure 23(b). There is only a small difference between the concentration dependence of $\kappa$ obtained from the time-resolved $(\bullet)$ and the stationary $(\star)$ experiments. The coincidence between this data is best at the highest concentration where the quenching is accomplished within the credibility time interval. The theoretical description of the whole concentration dependence is quite satisfactory and this is a great success of DET that it accounts for distant transfer.

Contrary to DET, the contact Smoluchowski-CollinsKimball approach is completely unable to fit the same data even at zero concentration. The ideal (IET) Stern-Volmer constant $\kappa_{0}=\kappa(0)$ is underestimated despite the usage of the same kinetic constant of the double channel reaction (50): $k_{a}=k_{0}+k_{1}$. This indicates that the reaction is under diffusional control when the contact $\kappa_{0}^{\text {con }} \approx k_{D}=4 \pi \sigma D$ is smaller than its distant analog $\kappa_{0} \approx 4 \pi R_{Q} D$, because

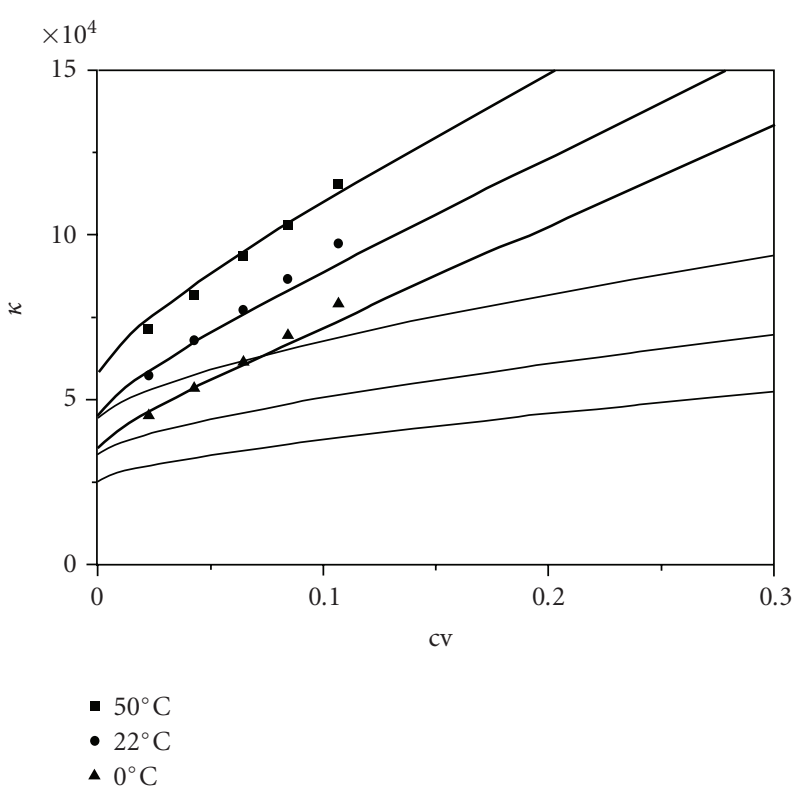

Figure 22: The experimental concentration dependence of the Stern-Volmer constant for three different temperatures (points) fitted by DET with the single-channel Marcus transfer rate (thick lines). The thin lines represent the contact analogs of the above curves at the same temperatures (decreasing from top to bottom). From [47].

$R_{Q}>\sigma$. Indeed, the accurately calculated $R_{Q}$ appears to be $8.6 \AA$ while $\sigma=4 \AA$ [48].

There is also an independent confirmation that this is actually the case. The Per/TCNE reaction is the most exergonic among the systems collected by Rehm and Weller when they inspected the applicability of the FEG law [88]. As was pointed out by Marcus and Siders [89], the top of the 


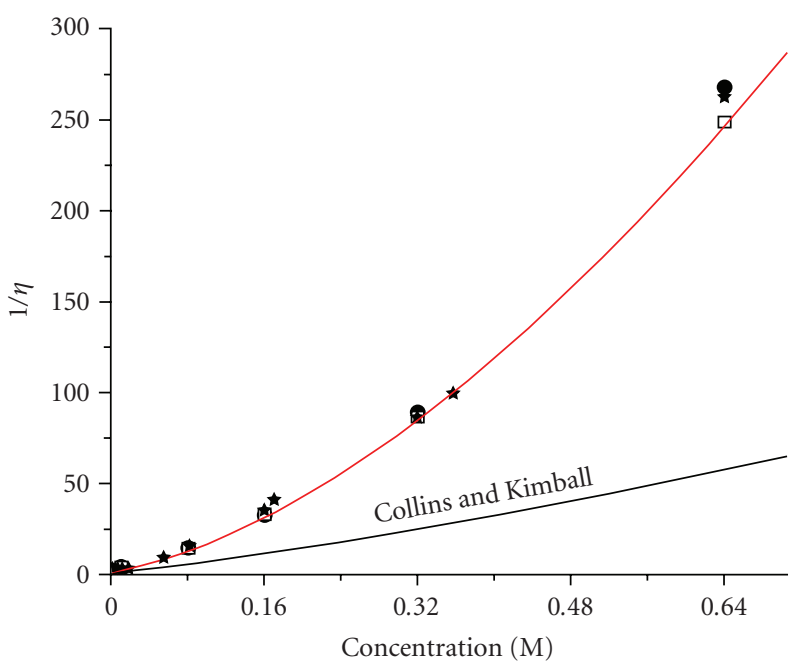

(a)

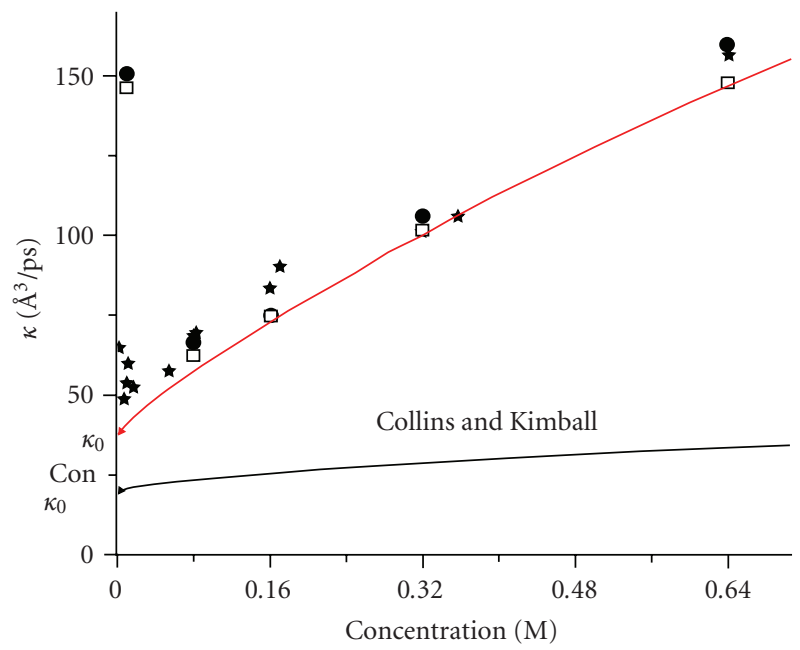

(b)

Figure 23: The non-linear Stern-Volmer law (a) and the Stern-Volmer constants (b) obtained from the time-resolved $(\bullet)$ and stationary $(\star)$ data in comparison with the theoretical predictions, following from the quenching kinetics integrated over the credibility intervals $(\square)$ and up to infinite time (red lines). From [48].

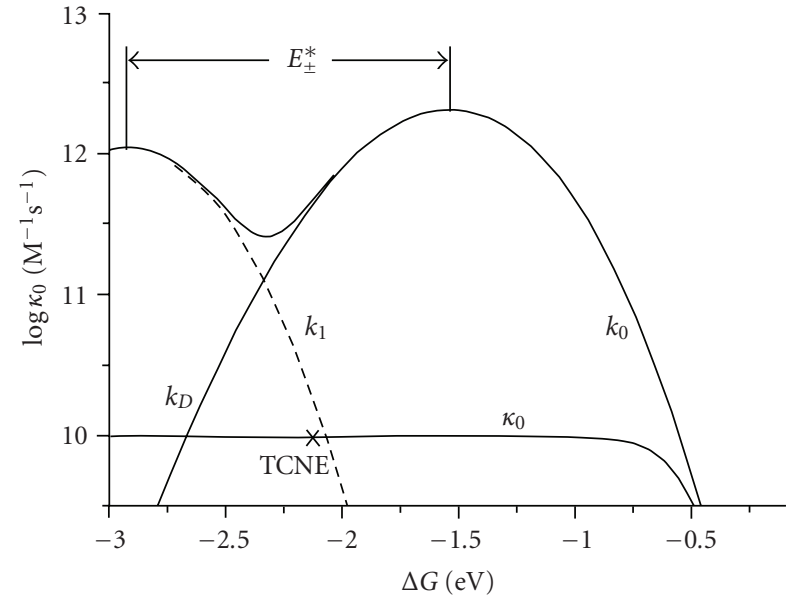

Figure 24: The Rehm-Weller plot of the Stern-Volmer constant of the double channel ionization, $\kappa_{0}(\Delta G)$, with a cross $(\times)$ marking the position of the Per/TCNE system. The bell-shaped FEG dependencies of the partial kinetic constants of the ground state and excited ions production, $k_{0}$ and $k_{1}$, split on the value of the ion pair excitation energy $E_{ \pm}^{*}$. From [95].

parabolic FEG dependence has to be cut by the diffusional plateau: $\kappa_{0} \approx k_{D}$. The Per/TCNE system is located at the left edge of this plateau, confusing everybody (Figure 24). There the descending branch representing the inverted region was expected to be detected instead of a plateau. A number of explanations were proposed for this paradox [37, 90-94] but nobody took the liberty of suggesting that the most exergonic ionization is still so fast that the reaction remains under diffusional control.

After fitting in [48] the double-channel ionization kinetics, $N_{A}^{*}(t)$, at all times, we needed no suggestions: with an obtained set of parameters, the partial constants $k_{0}=$ $\int W_{0} d^{3} r$ and $k_{1}=\int W_{1} d^{3} r$ were calculated without adding any new fitting parameters [95]. Not only the sum of these constants but even each of them appear to be larger than $k_{D}$ at the point where Per/TCNE is located (Figure 24). Such a relationship proves that the ionization is really under diffusion control in this system. This is the final resolution of the old paradox.

If there is no time-resolved data about $k_{A}(t)$ in the shortest time diapason but the good value of $k=k_{A}(\infty)=$ $4 \pi R_{Q} D$ is obtained, then the quantities $R_{Q}$ and $D$ can be separated, fitting the theory to the Stern-Volmer constants available from the stationary experiments. This program was successfully realized in a very recent paper [96]. The lack of information was filled in by relating the Stern-Volmer constant $\kappa(c)$ to the DET rate constant, $k_{A}(t)$ :

$$
\frac{1}{1+c \kappa \tau_{A}}=\int_{0}^{\infty} e^{-c \int_{0}^{t} k_{A}\left(t^{\prime}\right) d t^{\prime}-t / \tau_{A}} d t / \tau_{A}
$$

Unfortunately, the theoretical $\kappa(c, D)$ dependence that has to be fitted to the experimental data strongly depends on how accurately $k_{A}(t)$ is related to the transfer rate, $W_{A}(r)$, and encounter diffusion $D$ considered as a fitting parameter. This relationship is generally more complex than (5):

$$
k_{A}(t)=\int W_{A}(r) n(r, t) d^{3} r
$$

where $n(r, t)=\nu(r, t) e^{t / \tau_{A}}$ obeys the auxiliary diffusional equation alternative to $(6)$ :

$$
\dot{n}(r, t)=-W_{A} n(r, t)+\frac{1}{r^{2}} \frac{\partial}{\partial r} r^{2} D(r) g(r) \frac{\partial}{\partial r} \frac{n(r, t)}{g(r)} .
$$

The latter has to be solved with the reflecting boundary condition and initial condition $n(r, 0)=g(r)$. Here $g(r)$ is 
the equilibrium pair distribution function accounting for the internal structure of the liquid. Besides, the hydrodynamic effect is taken into account, which results in the space dependence of the diffusion coefficient at short distances between reactants. This dependence is represented by the simple expressions taken from either the Northrup and Hynes or Deutch and Felderhof models [96]:

$$
D_{\mathrm{NH}}(r)=D\left(1-\frac{1}{2} e^{-(r-\sigma) / \sigma}\right) \quad \text { or } D_{\mathrm{DF}}(r)=D\left(1-\frac{3 \sigma}{4 r}\right) .
$$

In fact, the authors of [96] tested 6 different models of $k_{A}(t)$ calculations (with and without accounting for $g(r)$ and $D(r)$ ) and selected only two of them which account simultaneously for the liquid structure and the space dependence of $D(r)$ (one or another). 150 experimental data points for $\kappa(c, D)$ were obtained from the fluorescence quantum yield studied in the 8 solvents of different compositions (viscosities). Not only the rate parameters, $L$ and $V_{0}$, were found from best fitting the theory to this database, but also the diffusion coefficient was allowed to vary individually for each solution, during the fitting procedure. The obtained relationships between diffusion and viscosity, $D(\eta)$, for the models (128) were shown to be very close to the StokesEinstein models for the stick and slip friction coefficient. Specifying $D$ in this way, the authors of [96] got $R_{Q}=k / 4 \pi D$ from $k=k_{A}(\infty)$ and demonstrated that such obtained $R_{Q}(D)$ dependence is in good agreement with its theoretical prediction having the asymptotes (124).

5.3. Products Accumulation. If the transfer occurs only at contact, then the products of the reaction (say RIPs) also appear only there, creating the contact initial condition for the subsequent geminate evolution. In contrast, the remote transfer proceeding from any distance creates the broad distribution of charged products around the contact that can be specified by means of DET. For this goal the differential theory should be completed by adding an equation for the free ion concentration to (3):

$$
\begin{aligned}
\dot{N}_{A}^{*} & =-k_{A}(t) c N_{A}^{*}-\frac{N_{A}^{*}}{\tau_{A}}, \\
\dot{P} & =k_{A}(t) c N_{A}^{*},
\end{aligned}
$$

where $k_{A}(t)$ was defined in (5). This set of equations is the irreversible analog of the IET equations (31).

The total number of transfer products $P$ is related to their pair distribution as usual:

$$
P(t)=c \int m(r, t) d^{3} r
$$

It follows from (129b) and (5) that

$$
\begin{gathered}
\dot{m}-W_{A} v e^{t / \tau_{A}} N_{A}^{*}=0, \\
m(r, t)=W_{A}(r) \int_{0}^{t} v\left(r, t^{\prime}\right) e^{t^{\prime} / \tau_{A}} N_{A}^{*}\left(t^{\prime}\right) d t^{\prime} .
\end{gathered}
$$

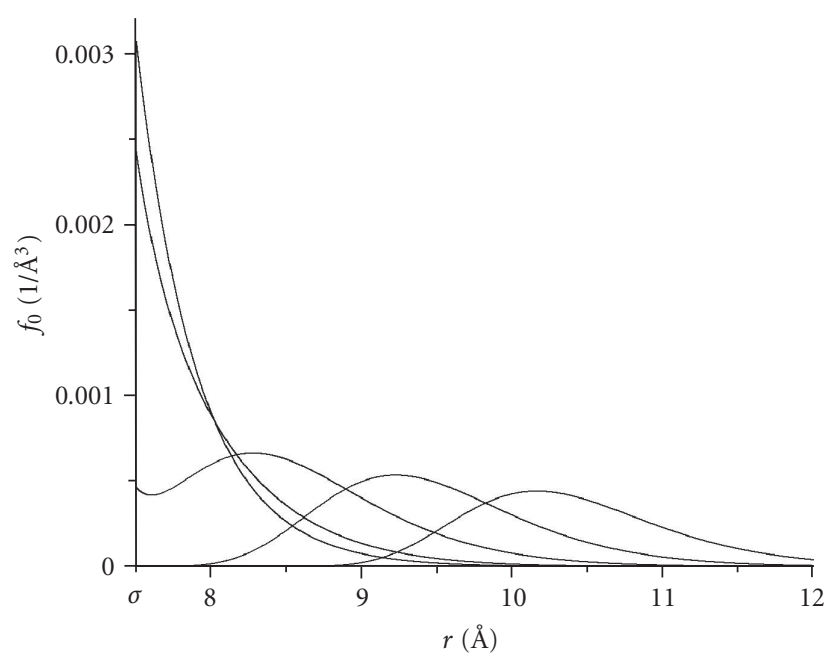

FIgURE 25: The distribution of ion pairs resulting from the ionization with exponential transfer rate $\left(W_{c}=29.12 \mathrm{~ns}^{-1}\right.$, $l=0.81 \AA$ ) at different encounter diffusion of neutral precursor: $D=10^{-4}, 10^{-6}, 10^{-7}, 10^{-8}, 10^{-9} \mathrm{~cm}^{2} / \mathrm{s}$ (from left to right).

The final distribution of charged products and its normalized version are

$$
\begin{gathered}
m(r, \infty)=W_{A}(r) \int_{0}^{\infty} v(r, t) e^{t / \tau_{A}} N_{A}^{*}(t) d t=m_{0}(r), \\
f_{0}=\frac{m_{0}(r)}{\int m_{0}(r) d^{3} r} .
\end{gathered}
$$

This distribution is weakly concentration dependent but changes dramatically with solvent viscosity (diffusion).

The family of such distributions obtained for exponential $W_{A}(r)$ from (119) is shown in Figure 25. At slow diffusion the distribution looks like a quasisymmetric hill shifted out of contact. With increasing diffusion it draws nearer to the contact, only slightly changing shape until ionization remains diffusional. As soon as it becomes kinetic the distribution approaches the contact, taking the shape of the ionization rate (exponential in our example). This phenomenon peculiar for only remote transfer was first studied theoretically $[97,98]$ and then specified in a few experimental works $[99,100]$.

5.4. Charge Separation. However, the accumulated ions are not frozen but start to separate and recombine immediately after their appearance at starting point $r_{0}$. Their total number, summarizing the contributions from any $r_{0}$, decreases with time as

$$
P(t)=c \int \Omega\left(t \mid r_{0}\right) m\left(r_{0}, t\right) d^{3} r_{0} .
$$

Here $m\left(r_{0}, t\right)$ is defined in (131), while $\Omega\left(t \quad \mid r_{0}\right)=$ $\int m\left(r, r_{0}, t\right) d^{3} r$ is the share of ions starting from distance $r_{0}$ but having escaped recombination up to time $t$. The latter obeys the conjugate diffusional equation $[16,101]$ for 
geminate recombination with distant tunneling rate $W_{R}(r)$ and ion diffusion coefficient $\widetilde{D}$ :

$$
\frac{\partial}{\partial t} \Omega(t \mid r)=-W_{R}(r) \Omega(t \mid r)+\frac{\widetilde{D} e^{-r_{c} / r}}{r^{2}} \frac{\partial}{\partial r} r^{2} e^{r_{c} / r} \frac{\partial}{\partial r} \Omega(t \mid r),
$$

where $r_{c}=e^{2} / \epsilon T$ is the Onsager radius of the Coulomb well in the solvent with dielectric constant $\epsilon$. Equation (134) should be solved with the following initial and boundary conditions:

$$
\begin{array}{r}
\Omega(0 \mid r)=1, \\
\left.\frac{\partial \Omega(t \mid r)}{\partial r}\right|_{r=\sigma}=0, \\
\Omega(t \mid \infty)=1 .
\end{array}
$$

The quantum yield of charge separation from the particular starting distance $r_{0}$ is

$$
\varphi\left(r_{0}\right)=\Omega\left(\infty \mid r_{0}\right)=\frac{1}{1+Z\left(r_{0}\right) / \widetilde{D}},
$$

while the total yield of the free ions is

$$
\phi=P(\infty)=c \int \varphi\left(r_{0}\right) m_{0}\left(r_{0}\right) d^{3} r_{0}=\psi \bar{\varphi},
$$

where the yield of ionization

$$
\psi=c \int m_{0}\left(r_{0}\right) d^{3} r_{0}=1-\eta
$$

and the charge separation yield

$$
\bar{\varphi}=\int \varphi\left(r_{0}\right) f_{0}\left(r_{0}\right) d^{3} r_{0}=\frac{1}{1+Z / \widetilde{D}} .
$$

The last expressions in (136) and (139) relate the charge separation yields to the partial and total recombination efficiencies, $Z\left(r_{0}\right)$ and $Z$. Such a diffusional dependence of the yields is natural for the archaic "exponential model" which implied that $Z=z=$ const $[9,16]$. Though incorporated into the encounter theory, the recombination efficiency $Z\left(r_{0}\right)$ becomes the function of not only $\widetilde{D}$ but also $r_{0}$, while $Z$ appears to be dependent on both $\widetilde{D}$ and $D$.

Two factors determine the type of this dependence: the shape of the recombination layer and the location of the starting point. The layer may be adjacent to the contact or shifted out depending on the form of the distant recombination rate $W_{R}(r)$, while $r_{0}$ can be located either inside or outside this layer or be distributed around it in accordance with $f_{0}\left(r_{0}\right)$.

For the exponential and rectangular models of $W_{R}(r)$, the diffusional dependence of $Z\left(r_{0}\right)$ is demonstrated in Figure 26. For starts taken from contact (or deeply inside the reaction zone), the efficiency of recombination monotonously decreases with diffusion, separating the ions. In contrast, the same diffusion accelerates the recombination bringing together the ions born outside the recombination zone. If initially ions were born inside the reaction layer but close to its outer boundary, then the initial inhibition of recombination finally gives way to its acceleration. Only as $\widetilde{D} \rightarrow \infty$ the efficiency of recombination, $Z_{\infty}$, is the same wherever the ions start from.

As a matter of fact, one cannot change the ion diffusion by varying solvent viscosity but keeping fixed and unique the initial separation $r_{0}$. The diffusion of neutral reactants changes simultaneously with that of ions, $D \approx \widetilde{D} \propto 1 / \eta$, where $\eta$ is the viscosity varying with solvent composition or pressure. As a result, the distribution over $r_{0}$ gradually approaches the contact when viscosity decreases (Figure 25) and $\bar{r}_{0}=\int r_{0} f_{0}\left(r_{0}\right) d^{3} r_{0} \rightarrow \sigma$. The total efficiency $Z$ shown in Figure 27 is averaged over the current distributions and therefore depends not only on $\widetilde{D}$ but also on $D$. When the diffusion is slowest, the start is more remote than recombination and efficiency increases, with diffusion controlling the reaction. At faster diffusion the situation changes to the opposite and a further increase in diffusion reduces the recombination efficiency [102].

However, the maximum which separates these regions is not sometimes seen. The recombination layer having the shape of the Marcus rate (115) is broad and far from contact, if the recombination proceeds in the inverted Marcus region (Figure 18) but attached to contact and narrow in the normal Marcus region (Figure 17). In the former case the ascending branch of $Z(D)$ gives way to the descending one much earlier than in the latter and does not give at all if recombination is contact (Figure 27). Only in the latter case is the reaction always under classic diffusional control (dotted line). Otherwise efficiency passes through the maximum before approaching its kinetic (i.e., "exponential model") limit, $z$.

Unfortunately, just detecting the monotonously growing $Z(\widetilde{D})$ does not guarantee that the recombination is contact or at least controlled by diffusion. Simultaneously with diffusion (viscosity), the variation of solvent composition changes also $n$ and $\epsilon$, as well as the contact reorganization energy (117) determined by these parameters. As a result, $W_{R}(r)$ becomes different at different compositions, as well as the curves $Z(\widetilde{D})$, corresponding to the fixed $\lambda_{0}$ particular for the given composition. These curves are shown by dashed and dotted lines in Figure 28 for the highest and lowest values of $\lambda_{0}$ that were achieved in the system studied by Wolf et al. [103]. This was the ruthenium complex quenched by methylviologen $\left(\mathrm{MV}^{++}\right)$, according to the reaction scheme

$$
\begin{gathered}
\left(\mathrm{Ru}^{2+}\right)^{*}+\mathrm{MV}^{2+} \stackrel{W_{I}}{\longrightarrow}\left[\dot{\mathrm{Ru}}{ }^{3+} \ldots \dot{\mathrm{M}} \mathrm{V}^{+}\right] \longrightarrow \dot{\mathrm{R}} \mathrm{u}^{3+}+\dot{\mathrm{M}} \mathrm{V}^{+} \\
{\| W_{R}} \\
{\left[\mathrm{Ru}^{2+} \ldots \mathrm{MV}^{2+}\right]}
\end{gathered}
$$

This reaction was studied in ionic solutions, when an electrolyte is dissolved in an inert solvent, like acetonitrile. The viscosity changed with mixture composition.

It can be seen easily from Figure 28 that both curves, calculated at fixed $\lambda_{0}$, pass through the maximum and their 


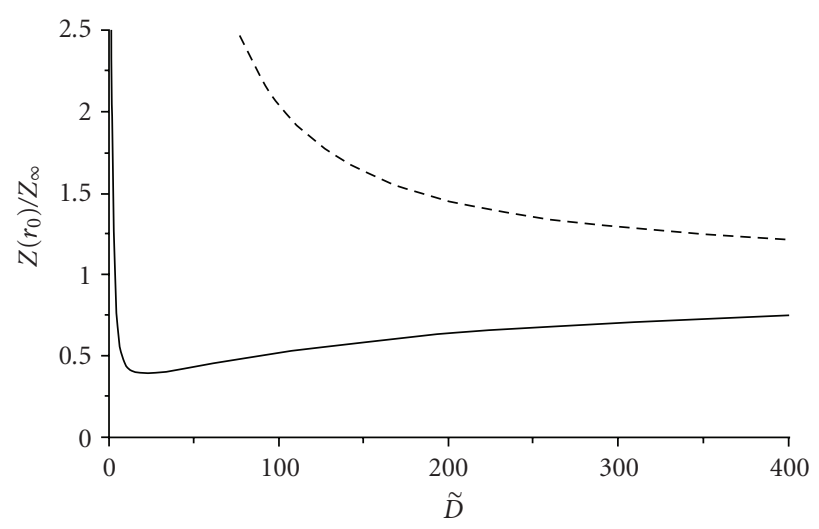

(a)

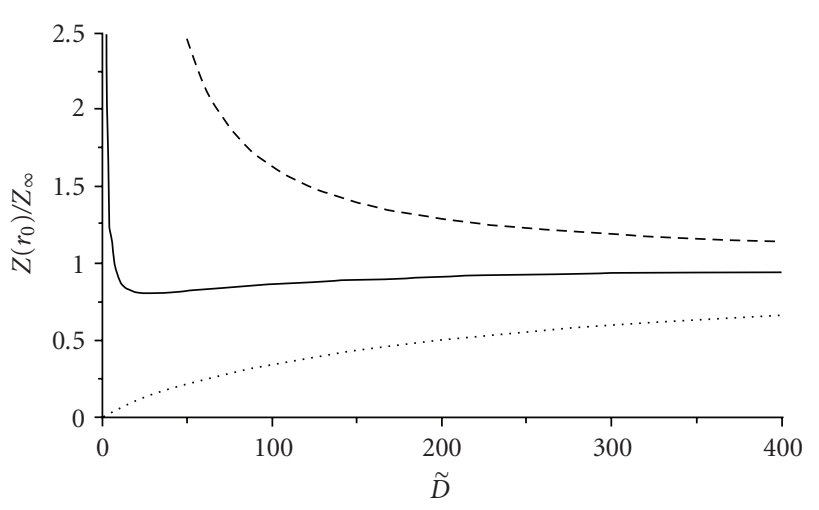

(b)

Figure 26: The partial efficiency of geminate recombination, $Z\left(r_{0}\right)$, related to its value at infinite diffusion, $Z_{\infty}$, for the exponential (a) and rectangular (b) models of the reaction rate. Contact starts (dashed lines), non-contact starts from reaction zones (solid lines) and start from outside of the rectangular zone (dotted line).

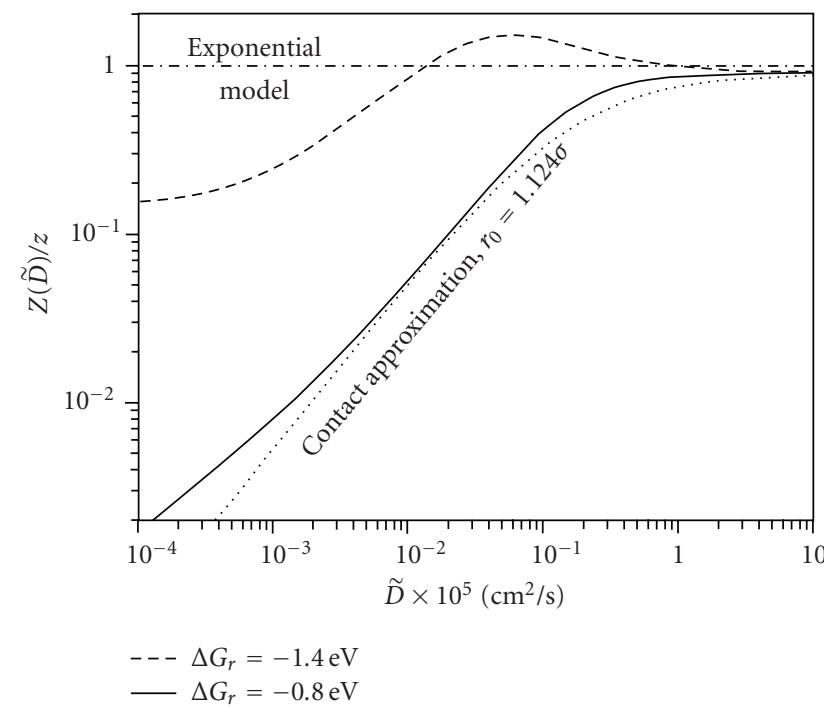

FIGURE 27: The diffusional dependence of the recombination efficiency $Z$ in the contact approximation (dotted line) at starting distance $r_{0}=1.124 \sigma$ and the same for the remote recombination in the normal (solid line) and inverted (dashed line) Marcus regions, in highly polar solvents. The horizontal dashed-dotted line represents the exponential model result, $Z=z=$ const. From [102].

viscosity dependence at faster diffusion is the opposite to that obtained experimentally in this system. The ascending branches of these curves can be attributed to the diffusion delivery of remote RIPs into the recombination zone but the experimental points are situated on the opposite, descending branches of them. Due to relatively fast diffusion, the forward electron transfer in this system is always under kinetic control and the RIPs are initially produced almost completely inside the recombination layer. Hence, the diffusional delivery of RIPs into this layer cannot be responsible for a pronounced increase in $Z$ with diffusion, as was thought earlier [104,

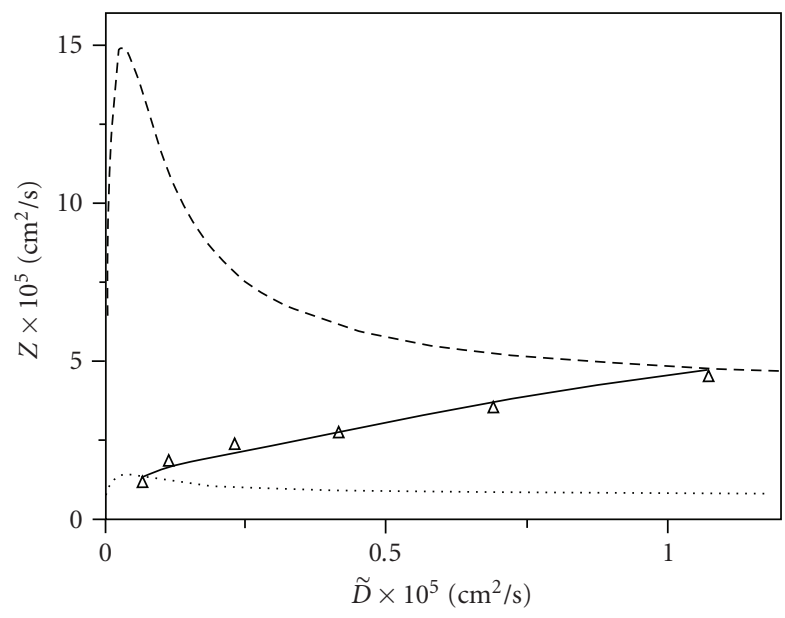

Figure 28: The experimental dependence of the recombination efficiency $Z$ on the diffusion coefficient $\tilde{D}$ shown by triangles. The solid line was calculated theoretically, taking into account that the reorganization energy changes along with diffusion. The dashed line corresponds to the calculation with fixed but maximal $\lambda_{0}$ reached at the fastest diffusion $\widetilde{D}=1.072 \times 10^{-5} \mathrm{~cm}^{2} / \mathrm{s}$. The dotted line was also calculated with a fixed but slowest diffusion $\widetilde{D}=6.67 \times 10^{-7} \mathrm{~cm}^{2} / \mathrm{s}$. From [102].

105]. It is due to the collateral change in $\lambda_{0}$ and $\tilde{D}$ with solvent composition. All experimental points belong to the descending branches of the curves which are intermediate between the dotted and dashed ones. The points shift up when viscosity goes down. The resulting increase in $Z$ just imitates the situation of diffusion-controlled recombination.

5.5. Unified Theory. More transparent results were obtained with dimethylsulfoxide-glycerol mixtures, which allow for a wide variation of solvent viscosity, without changing the parameters which affect $\lambda_{0}$. The system studied in 


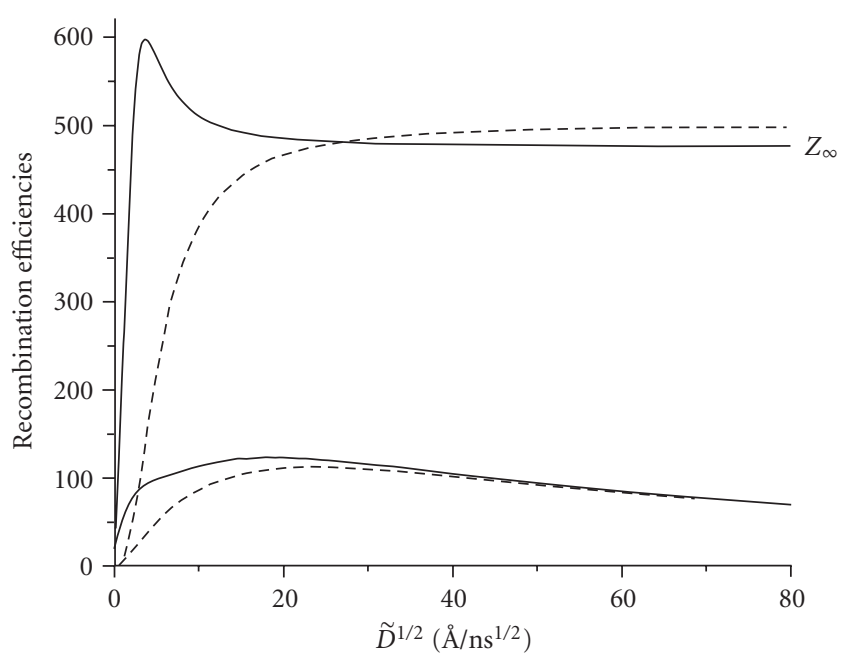

Figure 29: The total (above) and triplet (below) efficiencies of recombination obtained with contact (dashed lines) and exponential (solid lines) approximations of the recombination layer. From [106].

such mixtures was the photo-excited perylene quenched by aromatic amines:

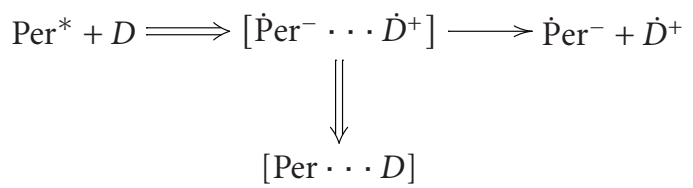

Being detailed, this reaction is represented by the scheme (93) accounting for the spin states of the reactants and RIPs. The spinless theory of Section 5.4 is inadequate to calculate the yields of the free ions and triplet products of the geminate recombination. The general unified theory (UT) should be employed for reaching this goal.

The original UT links together the geminate RIP recombination and RIP accumulation accounted for by the DET equation (131) $[97,98]$. Discriminating between singlet and triplet RIPs, one should consider their pair densities, $m_{S}(r, t)$ and $m_{T}(r, t)$, governed by a set of equations identical to [ 9 , equations (9.6)]:

$$
\begin{aligned}
\dot{m}_{S}-W_{A} \nu e^{t / \tau_{A}} N_{A}^{*}= & -W_{S}(r) m_{S}+\widetilde{D} \frac{1}{r^{2}} \frac{\partial}{\partial r} r^{2} e^{r_{c} / r} \frac{\partial}{\partial r} e^{-r_{c} / r} m_{S} \\
& +k_{s} m_{T}-3 k_{s} m_{S} \\
\dot{m}_{T}= & -W_{T}(r) m_{T}+\widetilde{D} \frac{1}{r^{2}} \frac{\partial}{\partial r} r^{2} e^{r_{c} / r} \frac{\partial}{\partial r} e^{-r_{c} / r} m_{T} \\
& -k_{s} m_{T}+3 k_{s} m_{S} .
\end{aligned}
$$

They have to be solved using the reflecting boundary conditions and assuming that initially all RIP states were empty: $m_{S}(0)=m_{T}(0)=0$. The right-hand side of the set (142) describes the geminate recombination/separation of RIPs subjected to spin conversion, while the left-hand side

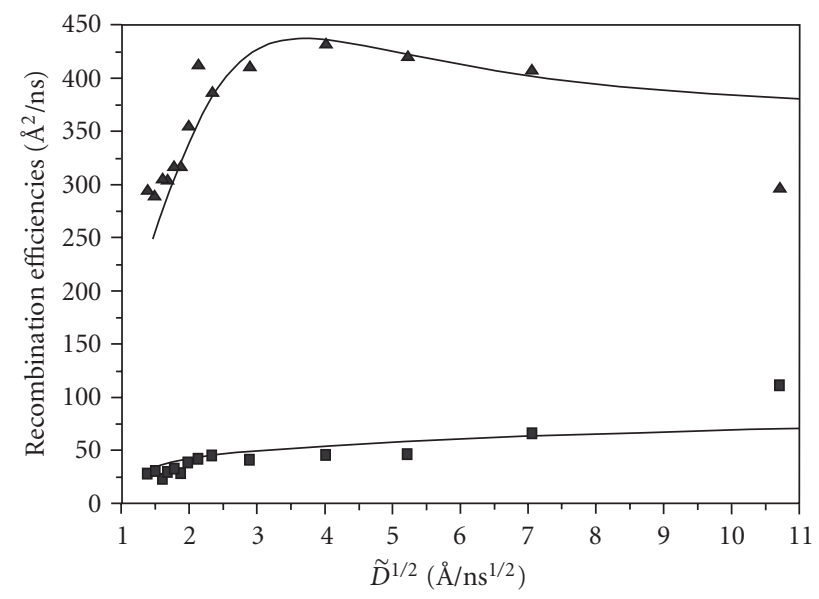

Figure 30: Fitting the theoretical efficiencies (solid lines) to the real experimental data for total $(\mathbf{\Delta})$ and triplet $(\boldsymbol{\square})$ recombination efficiencies, using the exponential models for both the ionization and recombination rates. Parameters obtained from the best fit are the following: $W_{i}=29.12 \mathrm{~ns}^{-1}, W_{s}=77 \mathrm{~ns}^{-1}=1.2 \cdot W_{t}$, $l_{I}=0.81 \AA, l_{R}=1.24 \AA, k_{s}=0.75 \mathrm{~ns}^{-1}, \sigma=7.5 \AA$. Reproduced from Figure 3.75 of the recent review [16].

accounts for their generation, via the same pumping term as in (131). This term composed from the DET quantities, $v$ and $N_{A}^{*}$, should be specified by the preliminary solution of DET equations (6) and (3). Using these solutions in the UT equations (142) and solving them, one obtains all that is necessary to calculate the quantum yields of the singlet (ground state) and triplet products:

$$
\begin{aligned}
& \phi_{S}\left(r^{\prime}\right)=\int_{0}^{\infty} \int W_{S}(r) m_{S}(r, t) d^{3} r d t=\psi \bar{\varphi}_{S}, \\
& \phi_{T}\left(r^{\prime}\right)=\int_{0}^{\infty} \int W_{T}(r) m_{T}(r, t) d^{3} r d t=\psi \bar{\varphi}_{T},
\end{aligned}
$$

where $\psi$ is the total RIP yield (138).

The yields of singlet and triplet products determine the efficiencies of recombination through these channels, $Z_{S}$ and $Z_{T}$ :

$$
\bar{\varphi}_{S}=\frac{Z_{S}}{Z+\widetilde{D}}, \quad \bar{\varphi}_{T}=\frac{Z_{T}}{Z+\widetilde{D}},
$$

while the charge separation yield is defined, as in (139):

$$
\bar{\varphi}=1-\bar{\varphi}_{S}-\bar{\varphi}_{T}=\frac{\widetilde{D}}{Z+\widetilde{D}},
$$

where $Z=Z_{S}+Z_{T}$. In view of the conservation law (145) it suffices to consider only two of the yields, $\bar{\varphi}$ and $\bar{\varphi}_{T}$, which are both available for the experimental detection.

In the contact approximation, the UT equations are available for the analytic solution obtained in [107, 108]. To do the same for remote transfer, the program Qyield developed by Dr. Krissinel (see http://www.fh.huji .ac.il/ krissinel/software.html) can be employed. If the exponential models for both the ionization and recombination rates are used, it should somehow reflect the fact 


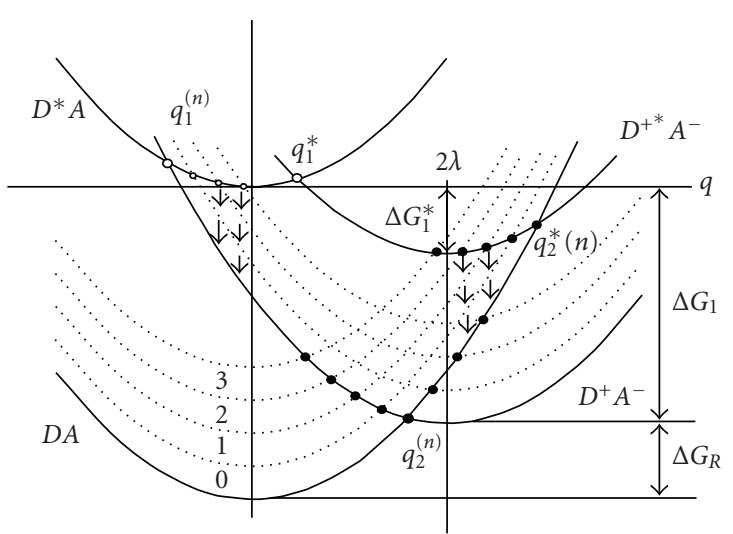

Figure 31: Ground and excited electronic states of the reactants, $D A$ and $D^{*} A$, and charged products, $D^{+} A^{-}$and $D^{+*} A^{-}$. The dotted lines represent vibrational excited states $(n=1,2,3)$, the crossing points for forward transfer are marked by circles ( 0 ) and for backward transfer by bullets $(\bullet)$. The arrows show the direction of the intra-molecular vibrational relaxation. From [49].

that the recombination rates are usually more extended, due to the larger exergonicity of the backward electron transfer. Accounting for this feature in our recent work we assumed [106] that $l_{R}>l_{I}$ thus makes the extension of recombination larger than ionization. Under this condition the total efficiency of remote recombination $Z$ is a nonmonotonous function of diffusion contrary to what is specific to the contact approximation (Figure 29). This is due to diffusional deceleration, following the diffusional acceleration of the recombination. At fastest diffusion the initial ion distribution coincides in shape with $W_{I}(r)$ which is narrower than $W_{S, T}(r)$. Therefore, the weaker the backward transfer is, the faster the ions get rid of the recombination layer interior. Passing the maximum, the total recombination efficiency $Z$ falls off with $\widetilde{D}$, approaching the plateau $Z_{\infty} \approx z=k_{c} / 4 \pi \sigma$.

The efficiency of triplet production also has a maximum, separating the diffusional control of recombination from the conversion control that turns $Z_{T}$ to zero, when too fast diffusion leaves no time for spin conversion. However, this maximum is usually not achievable in the experimentally available interval of diffusion variation. Fortunately, the principle difference between remote and contact recombination is seen even in these limits and is worthy of special attention.

The diffusional dependencies of both recombination efficiencies were subjected to experimental study in [108]. The extremum in $Z(\widetilde{D})$ first obtained there was later termed as Angulo effect. In Figure 30 we clearly see this feature. It gained a preliminary interpretation within the rectangular model of the recombination rate $[102,108]$, substituted later by the exponential one $[16,106]$. For fitting better the preexponent, $W_{s}=W_{S}(\sigma)$ was not assumed to be equal to $W_{t}=W_{T}(\sigma)$ but the relationship $W_{s}=1.2 \cdot W_{t}$ was preferred. Though at the greatest diffusion, the experimental points deviate a bit from the theoretical curves, all the rest are fitted quite well.

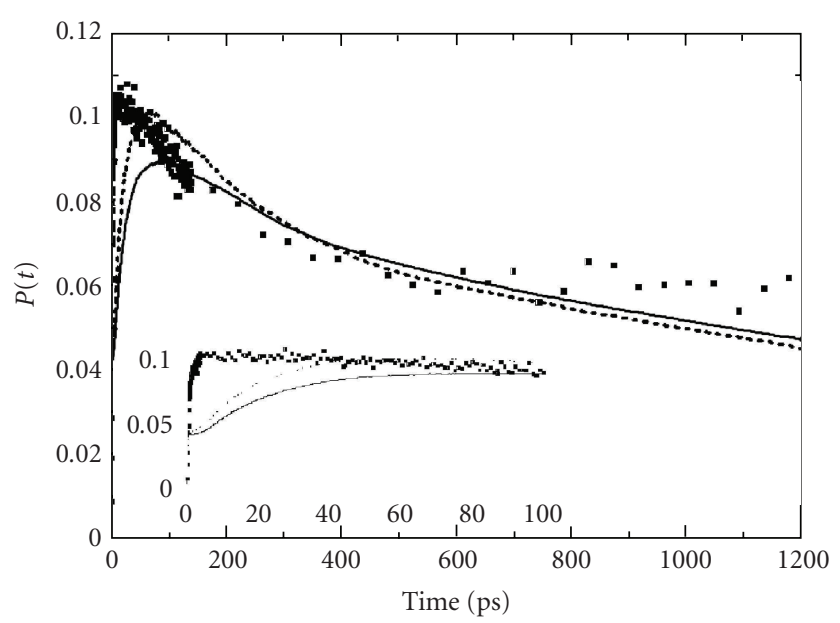

FIGURE 32: The best fit of the ion pairs accumulation/recombination kinetics (dots), using the multi-channel model of backward transfer with fixed $D$ and $\epsilon$ (solid lines) and accounting for their $r$ dependencies [49].

These results were essentially improved later on by the substitution of the real Marcus rates for their exponential approximations as well as the true (coherent) Hamiltonian description of spin-conversion for its rate (incoherent) model [109]. Assuming the HFI induced spin-conversion mechanism is responsible for the singlet-triplet mixing the true HFI constant $A$ was extracted from the best fitting. Neglecting the Coulomb interaction between solvated ions the viscosity dependence of the free ions and triplet quantum yields were specified as well as their magnetic field dependence. The only demerit was too large fitting value of $A \simeq 9 \mathrm{~ns}^{-1}=505 \times 10^{-4} \mathrm{~T}$ compared to that expected from ESR data $23 \times 10^{-4} \mathrm{~T}$. However, it has been shown recently that an accounting for the Coulomb interaction between the counter ions allows to get as good fitting but with much smaller $A$. Even in contact approximation, $A=47 \mathrm{~ns}^{-1}$ was obtained at moderate Onsager radius $r_{c}=12 \AA$ while for the remote electron transfer, $A=0.9 \mathrm{~ns}^{-1}$ is expected [75].

5.6. Adding the Reaction Coordinate. Usually all the encounter theories considering any kind of the electron transfer between the reactants ignore completely the internal structure of the latter, either electronic or vibrational. In fact, the intramolecular relaxation accompanied the electron transfer is responsible for the hot (transient) recombination and equilibration of transfer products, forming the transfer rates for subsequent thermal relaxation [49]. The thermal transfer rates of the forward and backward transfer, $W(\mathbf{r})=W(x, y, z)$, are usually the input data for the encounter theories. In actual fact, one should start earlier from the Hamiltonian and intraparticle relaxation that shape $W(\mathbf{r})$. Nobody can ignore the fact that the reaction develops not only in space but also along the reaction coordinate $q$, towards the term crossing points where it actually proceeds. In fact, only 4-dimensional $W(\mathbf{r}, \mathbf{q})$ should be used from the very beginning. 
The transient processes take some time and develop coincidentally with the encounter diffusion in $\mathbf{r}$ space. The conventional encounter theories presume the time separation: the faster formation of $W(r)$ compared to its modulation by space diffusion. This is never the case and the deep advancement of the reaction even during short times should not be excluded. Both processes were first considered simultaneously in DET assuming that the ionization proceeds with the rate $W(r, q)=A(r) \delta(q-$ $\left.q_{0}\right)$ in a single crossing point $q_{0}$ [110]. Next time the UT was extended correspondingly, taking into consideration not only double-channel ionization at a few crossing points $\left(q_{1}\right)$ but also the hot recombination in even more numerous intersections $\left(q_{2}\right)$ between the ground and excited states of Per/TCNE and $\mathrm{Per}^{+} / \mathrm{TCNE}^{-}$(Figure 31) [49]. It was shown experimentally that the hot recombination preceding the recombination of thermalized RIPs leaves for the latter only $10 \%$ of the initially produced ion pairs (Figure 32 ). This phenomenon is well proved by the 4-dimensional UT, although it predicts a bit slower accumulation than it is in reality. This demerit has been removed just recently by correcting the reorganization energy of the process making ion pair accumulation activationless and thus almost instantaneous [111]. Alternatively, it might be a consequence of the straightforward excitation of excited RIP as happens sometimes in stable complexes [112]. If this is an effect of light pumping it should be taken into account as it was done a few times for other purposes $[113,114]$.

The 4-dimensional IET, accounting for the reversibility of all the transitions included in Figure 31, was developed in [115]. Not only the reaction coordinate was included in this consideration but also the off-diagonal elements of transfer Hamiltonian instead of the rates of electron transfer. Quite recently, the charge transfer in the donoracceptor complex was studied, accounting for even two reaction coordinates: the fast and slow modes of the polar medium [116]. The implementation of this mechanism into the encounter theory will make it 5-dimensional, allowing one to correctly account for the biexponential dynamic solvent effect. The synthesis of the elementary act with an encounter diffusion of reactants within the framework of multidimensional encounter theory opens a new horizon for the chemical kinetics of liquid phase reactions.

\section{Conclusions}

There are two dyads of encounter theories: DET/UT and IET/MET. DET is applicable only to irreversible reactions but at any quencher concentrations, while UT is the most convenient approach for geminate recombination/separation of charged products. The integral theories are suitable for reversible reactions of any complexity, at the lowest (IET) and moderate (MET) concentrations. Neither of the encounter theories is universal but all of them together constitute a firm basis for studying any transfer reactions in liquids.

Both IET/MET and DET/UT are capable of accounting for the complex electronic structure of reactants, including their spin states. Any encounter theory considers energy and/or electron transfer as a remote reaction, resorting to the contact approximation only for simplification.

\section{Acknowledgment}

The author is sincerely grateful to Dr. A. Popov for placing at his disposal the colored panels of Figure 7 and Dr. K. L. Ivanov for helpful consultation.

\section{References}

[1] M. V. Smoluchowski, "Versuch einer mathematischen Theorie der Koagulationskinetik kolloider Lösungen," Zeitschrift für Physikalische Chemie, vol. 92, pp. 129-168, 1917.

[2] F. C. Collins and G. E. Kimball, "Diffusion-controlled reaction rates," Journal of Colloid Science, vol. 4, no. 4, pp. 425-437, 1949.

[3] N. N. Tunitskii and Kh. S. Bagdasar'yan,Optika i Spektroskopiya, vol. 15, p. 303, 1963.

[4] S. F. Kilin, M. S. Mikhelashvili, and I. M. Rozman, "On the transfer of electronic excitation energy in liquid solutions," Optika i Spektroskopiya, vol. 16, p. 576, 1964.

[5] I. I. Vasil'ev, B. P. Kirsanov, and V. A. Krongaus,Kinetika $i$ kataliz, vol. 5, p. 792, 1964.

[6] I. Z. Steinberg and E. Katchalski, "Theoretical analysis of the role of diffusion in chemical reactions, fluorescence quenching, and nonradiative energy transfer," The Journal of Chemical Physics, vol. 48, no. 6, pp. 2404-2410, 1968.

[7] A. B. Doktorov and A. I. Burshtein, "Quantum theory of distance transfer accelerated by diffusion," Soviet Physics JETP, vol. 41, p. 671, 1975.

[8] A. I. Burshtein, E. I. Kapinus, I. Yu. Kucherova, and V. A. Morozov, "Kinetics of long-range electron transfer: pheophytin a fluorescence quenching by toluquinone in liquid solutions," Journal of Luminescence, vol. 43, no. 5, pp. 291-299, 1989.

[9] A. I. Burshtein, "Unified theory of photochemical charge separation," in Advances in Chemical Physics, Volume 114, Advances in Chemical Physics, pp. 419-587, John Wiley \& Sons, New York, NY, USA, 2000.

[10] V. P. Sakun, "Intermolecular spin-spin interactions in liquids," Physica A, vol. 80, no. 2, pp. 128-148, 1975.

[11] A. B. Doktorov, "The impact approximation in the theory of bimolecular quasi-resonant processes," Physica A, vol. 90, no. 1, pp. 109-136, 1978.

[12] A. A. Kiprianov, A. B. Doktorov, and A. I. Burshtein, "Binary theory of dephasing in liquid solutions. II. Motiontransformed isotropic Raman spectra," Chemical Physics, vol. 76, no. 2, pp. 163-174, 1983.

[13] N. N. Lukzen, A. B. Doktorov, and A. I. Burshtein, "NonMarkovian theory of diffusion-controlled excitation transfer," Chemical Physics, vol. 102, no. 3, pp. 289-304, 1986.

[14] A. I. Burshtein and N. N. Lukzen, "Reversible reactions of metastable reactants," The Journal of Chemical Physics, vol. 103, no. 22, pp. 9631-9641, 1995.

[15] A. I. Burshtein and N. N. Lukzen, "Excitation trapping in liquid solutions," The Journal of Chemical Physics, vol. 105, no. 21, pp. 9588-9596, 1996.

[16] A. I. Burshtein, "Non-Markovian theories of transfer reactions in luminescence and chemiluminescence and photoand electrochemistry," in Advances in Chemical Physics, Volume 129, S. A. Rice, Ed., p. 105, John Wiley \& Sons, New York, NY, USA, 2004. 
[17] A. A. Kipriyanov, I. V. Gopich, and A. B. Doktorov, "A modification of non-Markovian encounter theory. I. Markovian description in non-Markovian theories," Chemical Physics, vol. 187, no. 3, pp. 241-249, 1994.

[18] A. A. Kipriyanov, I. V. Gopich, and A. B. Doktorov, "A modification of the non-Markovian encounter theory. III. Hopping and diffusion mechanisms of reactions," Chemical Physics, vol. 191, no. 1-3, pp. 101-118, 1995.

[19] A. A. Kipriyanov, I. V. Gopich, and A. B. Doktorov, "A manyparticle approach to the derivation of binary non-Markovian kinetic equations for the reaction $\mathrm{A}+\mathrm{B} \rightarrow \mathrm{B}$," Physica A, vol. 255, no. 3-4, pp. 347-405, 1998.

[20] I. V. Gopich, A. A. Kipriyanov, and A. B. Doktorov, "A manyparticle treatment of the reversible reaction $A+B \Leftrightarrow C+B$," The Journal of Chemical Physics, vol. 110, no. 22, pp. 1088810898, 1999.

[21] A. A. Kipriyanov, O. A. Igoshin, and A. B. Doktorov, "New approach to the derivation of binary non-Markovian kinetic equations," Physica A, vol. 268, no. 3-4, pp. 567-606, 1999.

[22] O. A. Igoshin, A. A. Kipriyanov, and A. B. Doktorov, "Manyparticle treatment of nonuniform reacting systems $A+B \rightarrow$ $C$ and $A+B \rightarrow C+D$ in liquid solutions," Chemical Physics, vol. 244, no. 2-3, pp. 371-385, 1999.

[23] A. A. Kipriyanov, O. A. Igoshin, and A. B. Doktorov, "The effect of chemical displacement of $B$ species in the reaction $A+B \rightarrow B$," Physica A, vol. 275, no. 1-2, pp. 99-133, 2000.

[24] K. L. Ivanov, N. N. Lukzen, A. B. Doktorov, and A. I. Burshtein, "Integral encounter theories of multistage reactions. I. Kinetic equations," The Journal of Chemical Physics, vol. 114, no. 4, pp. 1754-1762, 2001.

[25] K. L. Ivanov, N. N. Lukzen, A. B. Doktorov, and A. I. Burshtein, "Integral encounter theories of multistage reactions. II. Reversible inter-molecular energy transfer," The Journal of Chemical Physics, vol. 114, no. 4, pp. 1763-1774, 2001.

[26] K. L. Ivanov, N. N. Lukzen, A. B. Doktorov, and A. I. Burshtein, "Integral encounter theories of the multistage reactions. III. Reversible intramolecular energy transfer," The Journal of Chemical Physics, vol. 114, no. 13, pp. 5682-5690, 2001.

[27] J. Sung, J. Chi, and S. Lee, "Nonequilibrium distribution function theory of diffusion-influenced reversible energytransfer reactions," The Journal of Chemical Physics, vol. 111, no. 3, pp. 804-815, 1999.

[28] J. Sung and S. Lee, "Relations among the modern theories of diffusion-influenced reactions. I. Reduced distribution function theory versus memory function theory of Yang, Lee, and Shin," The Journal of Chemical Physics, vol. 111, no. 22, pp. 10159-10170, 1999.

[29] J. Sung and S. Lee, "Relations among the modern theories of diffusion-influenced reactions. II. Reduced distribution function theory versus modified integral encounter theory," The Journal of Chemical Physics, vol. 112, no. 5, pp. 21282138, 2000.

[30] S. Lee and M. Karplus, "Kinetics of diffusion-influenced bimolecular reactions in solution. I. General formalism and relaxation kinetics of fast reversible reactions," The Journal of Chemical Physics, vol. 86, no. 4, pp. 1883-1903, 1987.

[31] S. Lee and M. Karplus, "Kinetics of diffusion-influenced bimolecular reactions in solution. II. Effects of the gating mode and orientation-dependent reactivity," The Journal of Chemical Physics, vol. 86, no. 4, pp. 1904-1921, 1987.
[32] S. Lee, M. Yang, K. J. Shin, K. Y. Choo, and D. Lee, "Theory of diffusion-influenced fluorescence quenching: dependence of the Stern-Volmer curve on light intensity," Chemical Physics, vol. 156, no. 3, pp. 339-357, 1991.

[33] J. Sung, K. J. Shin, and S. Lee, "Effect of light intensity on the fluorescence quenching kinetics probed by frequencydomain fluorometry," The Journal of Chemical Physics, vol. 101, no. 9, pp. 7241-7247, 1994.

[34] I. V. Gopich and A. Szabo, "Kinetics of reversible diffusion influenced reactions: the self-consistent relaxation time approximation," The Journal of Chemical Physics, vol. 117, no. 2, pp. 507-517, 2002.

[35] S. Park, K. J. Shin, A. V. Popov, and N. Agmon, "Diffusioninfluenced excited-state reversible transfer reactions, $A^{*}+$ $B \rightleftharpoons C^{*}+D$, with two different lifetimes: theories and simulations," The Journal of Chemical Physics, vol. 123, no. 3, Article ID 034507, 14 pages, 2005.

[36] O. G. Berg, "On diffusion-controlled dissociation," Chemical Physics, vol. 31, no. 1, pp. 47-57, 1978.

[37] N. Agmon, "From energy profiles to structure-reactivity correlations," International Journal of Chemical Kinetics, vol. 13, no. 4, pp. 333-365, 1981.

[38] D. Huppert, E. Pines, and N. Agmon, "Long-time behavior of reversible geminate recombination reactions," Journal of the Optical Society of America B, vol. 7, no. 8, p. 1545, 1990.

[39] I. V. Gopich and N. Agmon, "Excited-state reversible geminate reaction. III. Exact solution for noninteracting partners," The Journal of Chemical Physics, vol. 110, no. 21, pp. 10433-10444, 1999.

[40] N. Agmon and I. V. Gopich, "Kinetic transition in excitedstate reversible reactions," Chemical Physics Letters, vol. 302, no. 5-6, pp. 399-404, 1999.

[41] A. I. Burshtein, "Reversible charge separation through exciplex formation," The Journal of Chemical Physics, vol. 117, no. 16, pp. 7640-7648, 2002.

[42] I. V. Gopich and A. I. Burshtein, "Inter-system crossing catalyzed by solute encounters," The Journal of Chemical Physics, vol. 109, no. 7, pp. 2833-2843, 1998.

[43] A. I. Burshtein, A. A. Neufeld, and K. L. Ivanov, "Reversible electron transfer in photochemistry and electrochemistry," The Journal of Chemical Physics, vol. 115, no. 6, pp. 26522663, 2001.

[44] K. L. Ivanov, “Theoretical treatment of reversible energy transfer reactions of metastable reactants: modification of the integral encounter theory," Chemical Physics, vol. 315, no. 3, pp. 303-318, 2005.

[45] P. A. Frantsuzov, O. A. Igoshin, and E. B. Krissinel, "Differential approach to the memory-function reaction kinetics," Chemical Physics Letters, vol. 317, no. 3-5, pp. 481-489, 2000.

[46] A. J. Lotka, "Undamped oscillations derived from the law of mass action," Journal of the American Chemical Society, vol. 42, no. 8, pp. 1595-1599, 1920.

[47] A. V. Popov, V. S. Gladkikh, and A. I. Burshtein, "SternVolmer law in competing theories and approximations," The Journal of Physical Chemistry A, vol. 107, no. 40, pp. 81778183, 2003.

[48] V. Gladkikh, A. I. Burshtein, G. Angulo, S. Pagès, B. Lang, and E. Vauthey, "Kinetics and yields of electron transfer in the inverted region," The Journal of Physical Chemistry A, vol. 108, no. 32, pp. 6667-6678, 2004.

[49] V. Gladkikh, A. I. Burshtein, S. V. Feskov, A. I. Ivanov, and E. Vauthey, "Hot recombination of photogenerated ion pairs," 
The Journal of Chemical Physics, vol. 123, no. 24, Article ID 244510, 11 pages, 2005.

[50] A. I. Burshtein, I. V. Gopich, and P. A. Frantsuzov, "Accumulation and distribution of energy quenching products," Chemical Physics Letters, vol. 289, no. 1-2, pp. 60-66, 1998.

[51] W. Naumann, N. V. Shokhirev, and A. Szabo, "Exact asymptotic relaxation of pseudo-first-order reversible reactions," Physical Review Letters, vol. 79, no. 16, pp. 3074-3077, 1997.

[52] A. I. Burshtein and P. A. Frantsuzov, "Universal binary theory of photochemical charge separation and distribution," The Journal of Chemical Physics, vol. 106, no. 10, pp. 3948-3955, 1997.

[53] A. I. Burshtein and K. L. Ivanov, "Reversible photoionization in liquid solutions," The Journal of Physical Chemistry A, vol. 105, no. 13, pp. 3158-3166, 2001.

[54] A. I. Burshtein and K. L. Ivanov, "The crucial role of triplets in photoinduced charge transfer and separation," Physical Chemistry Chemical Physics, vol. 4, no. 17, pp. 4115-4125, 2002.

[55] A. V. Popov and A. I. Burshtein, "Theories of reversible dissociation: a comparative study," The Journal of Physical Chemistry A, vol. 107, no. 45, pp. 9688-9694, 2003.

[56] A. I. Ivanov and A. I. Burshtein, "Luminescence Quenching by Reversible Ionization or Exciplex Formation/Dissociation," The Journal of Physical Chemistry A, vol. 112, no. 28, pp. 6392-6397, 2008.

[57] R. D. Mussell and D. G. Nocera, "Effect of long-distance electron transfer on chemiluminescence efficiencies," Journal of the American Chemical Society, vol. 110, no. 9, pp. 2764$2772,1988$.

[58] R. D. Mussell and D. G. Nocera, "Partitioning of the electrochemical excitation energy in the electrogenerated chemiluminescence of hexanuclear molybdenum and tungsten clusters," Inorganic Chemistry, vol. 29, no. 19, pp. 37113717, 1990.

[59] P. Szrebowaty and A. Kapturkiewicz, "Free energy dependence on tris (2, 2' -bipyridine)ruthenium(II) electrochemiluminescence efficiency," Chemical Physics Letters, vol. 328, no. 1-2, pp. 160-168, 2000.

[60] A. I. Burshtein, A. A. Neufeld, and K. L. Ivanov, "Fluorescence and phosphorescence resulting from electrochemical generation of triplet excitations," The Journal of Chemical Physics, vol. 115, no. 22, pp. 10464-10471, 2001.

[61] P. Jacques and X. Allonas, "Is $\Delta G_{\text {et }}$ really the main factor governing photoinduced electron transfer rates?" Journal of Photochemistry and Photobiology A, vol. 78, no. 1, pp. 1-5, 1994.

[62] A. I. Burshtein, "Quenching of singlets and triplets by reversible ionization followed by charge recombination," The Journal of Physical Chemistry A, vol. 110, no. 51, pp. 1366713675, 2006.

[63] G. Porcal, S. G. Bertolotti, C. M. Previtali, and M. V. Encinas, "Electron transfer quenching of singlet and triplet excited states of flavins and lumichrome by aromatic and aliphatic electron donors," Physical Chemistry Chemical Physics, vol. 5, no. 19, pp. 4123-4128, 2003.

[64] G. Angulo, G. Grampp, A. A. Neufeld, and A. I. Burshtein, "Delayed fluorescence due to annihilation of triplets produced in recombination of photo-generated ions," The Journal of Physical Chemistry A, vol. 107, no. 36, pp. 69136919, 2003.
[65] K. M. Salikhov, Yu. N. Molin, R. Z. Sagdeev, and A. L. Buchachenko, Spin Polarization and Magnetic Effects in Radical Reactions, Akademiai Kiado, Budapest, Hungary, 1984.

[66] D. Bürßner, H.-J. Wolff, and U. Steiner, "Magnetokinetic probing of extremely fast electron spin relaxation in paramagnetic ruthenium complexes," Zeitschrift für Physikalische Chemie B, vol. 182, p. 297, 1993.

[67] D. Bürßner, H.-J. Wolff, and U. Steiner, "Magnetic spin effects on photooxidation quantum yields of $\mathrm{Ru}^{\mathrm{II}}$-tris(bipyridine) type complexes in magnetic fields up to 17.5 tesla," Angewandte Chemie International Edition, vol. 33, no. 17, pp. 1772-1775, 1994.

[68] A. I. Burshtein, "Recombination and separation of photochemically created radical-ion pairs subjected to incoherent spin-conversion," Chemical Physics, vol. 323, no. 2-3, pp. 341-350, 2006.

[69] R. G. Mints and A. A. Pukhov, "The influence of paramagnetic impurities on magnetic effects in radical reactions," Chemical Physics, vol. 87, no. 3, pp. 467-472, 1984.

[70] V. S. Gladkikh and A. I. Burshtein, "Double-channel recombination of the radical pairs via incoherent $\delta g$-mechanism of spin-conversion," Chemical Physics, vol. 323, no. 2-3, pp. 351-357, 2006.

[71] Z. Schulten and K. Schulten, "The generation, diffusion, spin motion, and recombination of radical pairs in solution in the nanosecond time domain," The Journal of Chemical Physics, vol. 66, no. 10, pp. 4616-4634, 1977.

[72] E. V. Gorelik, N. N. Lukzen, R. Z. Sagdeev, and U. E. Steiner, "Application of integral encounter theory to account for the spin effects in radical reactions I. $\delta g$ and spin relaxation effects on recombination kinetics of free radicals," Chemical Physics, vol. 262, no. 2-3, pp. 303-323, 2000.

[73] V. S. Gladkikh and A. I. Burshtein, "Double-channel contact recombination of radical pairs subjected to spin conversion via the $\delta g$ mechanism," Journal of Physical Chemistry A, vol. 110, no. 10, pp. 3364-3376, 2006.

[74] N. N. Lukzen, J. B. Pedersen, and A. I. Burshtein, "Singlet and triplet products of the geminate recombination of a radical pair with a single magnetic nucleus $(I=1 / 2)$," Journal of Physical Chemistry A, vol. 109, no. 51, pp. 11914-11926, 2005.

[75] A. I. Ivanov and A. I. Burshtein, "The double-channel contact recombination and separation of geminate radical ion pairs in a Coulomb well," The Journal of Physical Chemistry A, vol. 112, no. 28, pp. 6392-6397, 2008.

[76] R. A. Marcus, "On the theory of oxidation-reduction reactions involving electron transfer. I," The Journal of Chemical Physics, vol. 24, no. 5, pp. 966-978, 1956.

[77] V. G. Levich and R. R. Dogonadze, "Teoriya bezyzluchatel'nykh elektronnykh perekhodov mezhdu ionami v rastvorakh," Doklady Akademii Nauk SSSR, vol. 124, pp. 123-126, 1959.

[78] V. G. Levich and R. R. Dogonadze, "Adiabatic theory of electron-transfer processes in solutions," Doklady Akademii Nauk SSSR, vol. 133, no. 1, pp. 159-161, 1960.

[79] V. S. Gladkikh, A. I. Burshtein, H. L. Tavernier, and M. D. Fayer, "Influence of diffusion on the kinetics of donoracceptor electron transfer monitored by the quenching of donor fluorescence," Journal of Physical Chemistry A, vol. 106, no. 30, pp. 6982-6990, 2002.

[80] A. I. Burshtein and P. A. Frantsuzov, "Noncontact diffusionaccelerated photoionization," Journal of Luminescence, vol. 51, no. 4, pp. 215-222, 1992. 
[81] A. I. Burshtein and N. V. Shokhirev, "Viscosity and free energy dependence of photochemical charge separation," Journal of Physical Chemistry A, vol. 101, no. 1, pp. 25-30, 1997.

[82] A. A. Neufeld, A. I. Burshtein, G. Angulo, and G. Grampp, "Viscosity dependence of geminate recombination efficiency after bimolecular charge separation," Journal of Chemical Physics, vol. 116, no. 6, pp. 2472-2479, 2002.

[83] H. L. Tavernier, M. M. Kalashnikov, and M. D. Fayer, "Photoinduced intermolecular electron transfer in complex liquids: experiment and theory," Journal of Chemical Physics, vol. 113, no. 22, pp. 10191-10201, 2000.

[84] B. Stevens and C. J. Biver III, "Parameterization of diffusioninfluenced intermolecular electron transfer in the static quenching limit," Chemical Physics Letters, vol. 226, no. 3-4, pp. 268-274, 1994.

[85] A. I. Burshtein and A. Yu. Sivachenko, "Photochemical accumulation and recombination of ion pairs undergoing the singlet-triplet conversion," Chemical Physics, vol. 235, no. 13, pp. 257-266, 1998.

[86] A. I. Burshtein, I. V. Khudyakov, and B. I. Yakobson, "Fast reactions between radicals. Pseudodiffusion control," Progress in Reaction Kinetics, vol. 13, no. 4, pp. 221-305, 1984.

[87] V. S. Gladkikh and A. I. Burshtein, "Photoionization affected by chemical anisotropy," Journal of Chemical Physics, vol. 126, no. 1, Article ID 014506, 7 pages, 2007.

[88] D. Rehm and A. Weller, "Kinetics of fluorescence quenching by electron and h-atom transfer," Israel Journal of Chemistry, vol. 8, pp. 259-271, 1970.

[89] R. A. Marcus and P. Siders, "Theory of highly exothermic electron transfer reactions," Journal of Physical Chemistry, vol. 86, no. 5, pp. 622-630, 1982.

[90] R. Ballardini, G. Varani, M. T. Indelli, F. Scandola, and V. Balzani, "Free energy correlation of rate constants for electron transfer quenching of excited transition metal complexes," Journal of the American Chemical Society, vol. 100, no. 23, pp. 7219-7223, 1978.

[91] K. Kikuchi, Y. Takahashi, T. Katagiri, T. Niwa, M. Hoshi, and T. Miyashi, "A critical consideration on the lack of inverted region in the Rehm-Weller plot for electron-transfer fluorescence quenching," Chemical Physics Letters, vol. 180, no. 5, pp. 403-408, 1991.

[92] M. Tachiya and S. Murata, "New explanation for the lack of the inverted region in charge separation reactions," Journal of Physical Chemistry, vol. 96, no. 21, pp. 8441-8444, 1992.

[93] C. Turró, J. M. Zaleski, Y. M. Karabatsos, and D. G. Nocera, "Bimolecular electron transfer in the marcus inverted region," Journal of the American Chemical Society, vol. 118, no. 25, pp. 6060-6067, 1996.

[94] S. Yasui, M. Tsujimoto, K. Itoh, and A. Ohno, "Quenching of a photosensitized dye through single-electron transfer from trivalent phosphorus compounds," Journal of Organic Chemistry, vol. 65, no. 15, pp. 4715-4720, 2000.

[95] A. I. Burshtein and A. I. Ivanov, "A diffusional alternative to the Marcus free energy gap law," Physical Chemistry Chemical Physics, vol. 9, no. 3, pp. 396-400, 2007.

[96] A. Rosspeintner, D. R. Kattnig, G. Angulo, S. Landgraf, G. Grampp, and A. Cuetos, "On the coherent description of diffusion-influenced fluorescence quenching experiments," Chemistry-A European Journal, vol. 13, no. 22, pp. 64746483, 2007.
[97] A. I. Burshtein, "Geminate recombination after binary photoionization," Chemical Physics Letters, vol. 194, no. 3, pp. 247-251, 1992.

[98] R. C. Dorfman and M. D. Fayer, "The influence of diffusion on photoinduced electron transfer and geminate recombination," The Journal of Chemical Physics, vol. 96, no. 10, pp. 7410-7422, 1992.

[99] S. Murata and M. Tachiya, "Transient effect in fluorescence quenching by electron transfer. 3. Distribution of electron transfer distance in liquid and solid solutions," Journal of Physical Chemistry, vol. 100, no. 10, pp. 4064-4070, 1996.

[100] S. Murata and M. Tachiya, "Electron transfer reactions studied through the transient effect in fluorescence quenching," Journal de Chimie Physique et de Physico-Chimie Biologique, vol. 93, no. 10, pp. 1577-1590, 1996.

[101] N. N. Korst, "Semiclassical theory of spin relaxation in media with a high viscosity," Theoretical and Mathematical Physics, vol. 6, no. 2, pp. 196-205, 1971.

[102] A. I. Burshtein and A. A. Neufeld, "Separation of photogenerated radical ion pairs in viscous solutions," Journal of Physical Chemistry B, vol. 105, no. 49, pp. 12364-12373, 2001.

[103] H.-J. Wolff, D. Bürßner, and U. E. Steiner, "Spin-orbit coupling controlled spin chemistry of $\mathrm{Ru}(\mathrm{bpy})_{3}^{2+}$ photooxidation: detection of strong viscosity dependence of in-cage backward electron transfer rate," Pure and Applied Chemistry, vol. 67, no. 1, pp. 167-174, 1995.

[104] E. B. Krissinel, A. I. Burshtein, N. N. Lukzen, and U. E. Steiner, "Magnetic field effect as a probe of distancedependent electron transfer in systems undergoing free diffusion," Molecular Physics, vol. 96, no. 7, pp. 1083-1097, 1999.

[105] A. I. Burshtein, E. B. Krissinel, and U. E. Steiner, "Diffusion, spin and reaction control in geminate reverse electron transfer," Physical Chemistry Chemical Physics, vol. 3, no. 2, pp. 198-203, 2001.

[106] V. S. Gladkikh, G. Angulo, and A. I. Burshtein, "Production of free radicals and triplets from contact radical pairs and from photochemically generated radical ions," Journal of Physical Chemistry A, vol. 111, no. 18, pp. 3458-3464, 2007.

[107] A. I. Burshtein and E. B. Krissinel, "Photochemical charge separation suppressed by spin conversion," Journal of Physical Chemistry A, vol. 102, no. 5, pp. 816-824, 1998.

[108] V. S. Gladkikh, A. I. Burshtein, G. Angulo, and G. Grampp, "Quantum yields of singlet and triplet recombination products of singlet radical ion pairs," Physical Chemistry Chemical Physics, vol. 5, no. 12, pp. 2581-2588, 2003.

[109] D. V. Dodin, A. I. Ivanov, and A. I. Burshtein, "Noncontact bimolecular photoionization followed by radical-ions separation and their geminate recombination assisted by coherent HFI induced spin-conversion," Journal of Physical Chemistry A, vol. 112, no. 5, pp. 889-897, 2008.

[110] A. A. Zharikov and A. I. Burshtein, "Nonlocal ionization in encounter theory," The Journal of Chemical Physics, vol. 93, no. 8, pp. 5573-5579, 1990.

[111] A. I. Burshtein, S. V. Feskov, A. I. Ivanov, and E. Vauthey, (work in progress).

[112] S. V. Feskov, V. S. Gladkikh, and A. I. Burshtein, "Kinetics of non-thermal electron transfer controlled by the dynamical solvent effect," Chemical Physics Letters, vol. 458, no. 1-3, pp. 71-75, 2008.

[113] O. A. Igoshin and A. I. Burshtein, "Impurity quenching of fluorescence in intense light. Violation of the Stern-Volmer law," Journal of Chemical Physics, vol. 112, no. 24, pp. 10930 10940, 2000. 
[114] O. A. Igoshin and A. I. Burshtein, "Quenching of fluorescence by irreversible energy transfer at arbitrary strong pumping light," Journal of Luminescence, vol. 92, no. 1-2, pp. 123-132, 2000.

[115] S. V. Feskov, A. I. Ivanov, and A. I. Burshtein, "Integral encounter theory of strong electron transfer," Journal of Chemical Physics, vol. 122, no. 12, Article ID 124509, 11 pages, 2005.

[116] S. V. Feskov, V. N. Ionkin, and A. I. Ivanov, "Effect of high-frequency modes and hot transitions on free energy gap dependence of charge recombination rate," Journal of Physical Chemistry A, vol. 110, no. 43, pp. 11919-11925, 2006. 


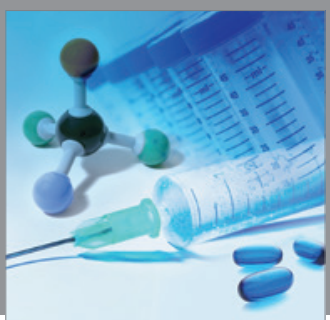

International Journal of

Medicinal Chemistry

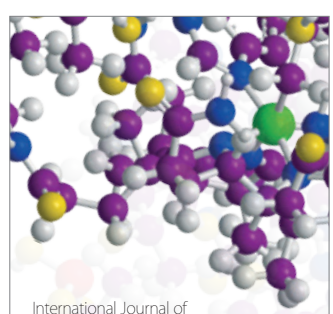

Carbohydrate Chemistry

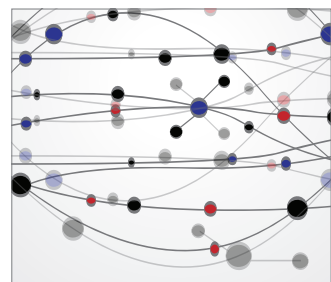

The Scientific World Journal
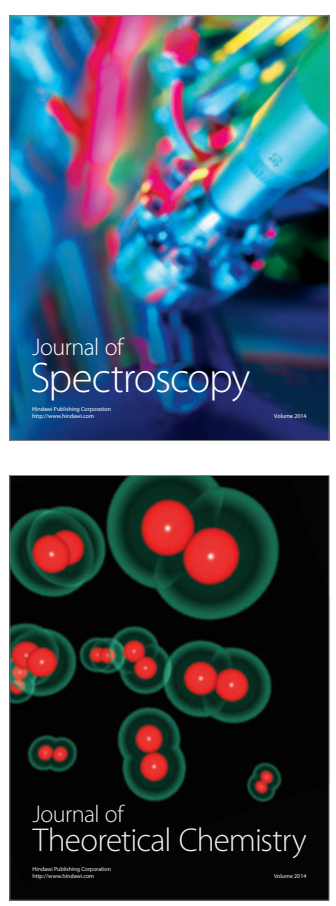
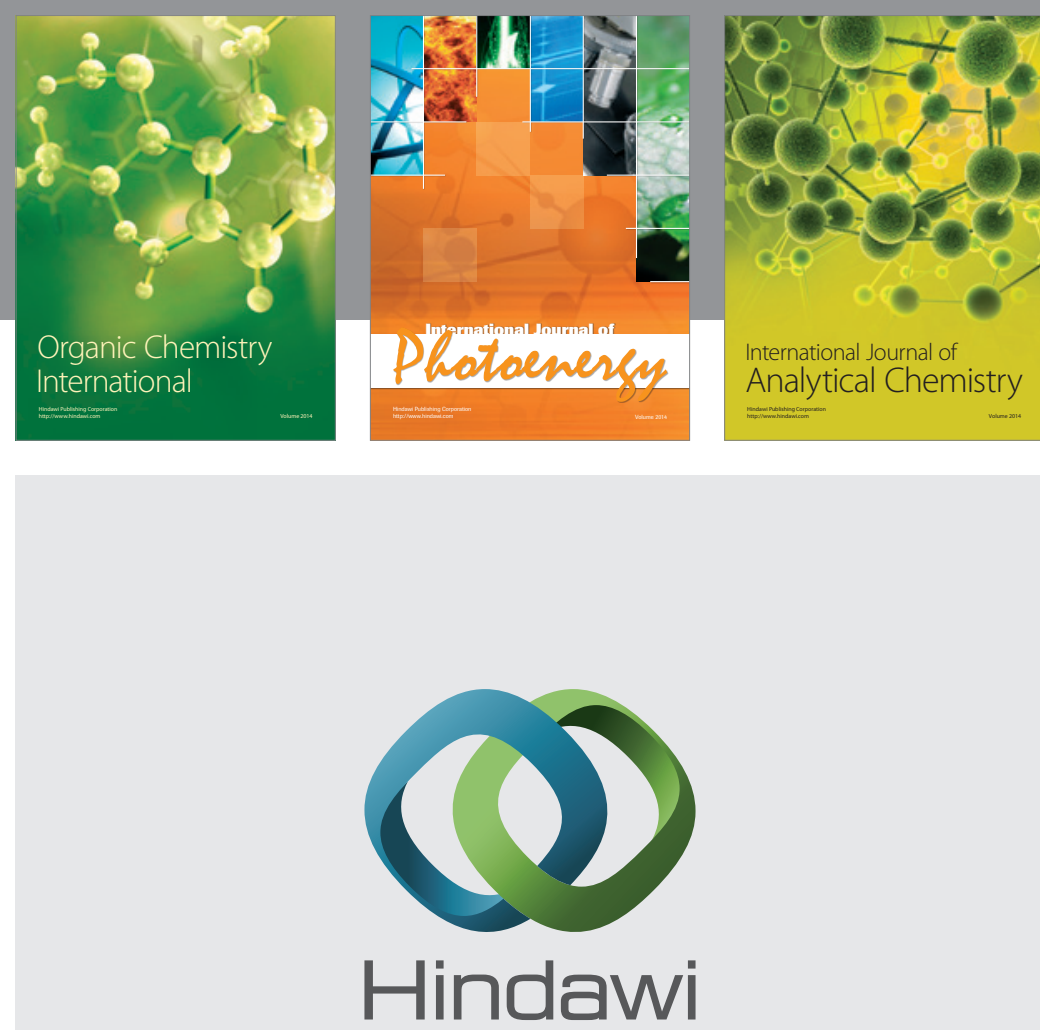

Submit your manuscripts at

http://www.hindawi.com
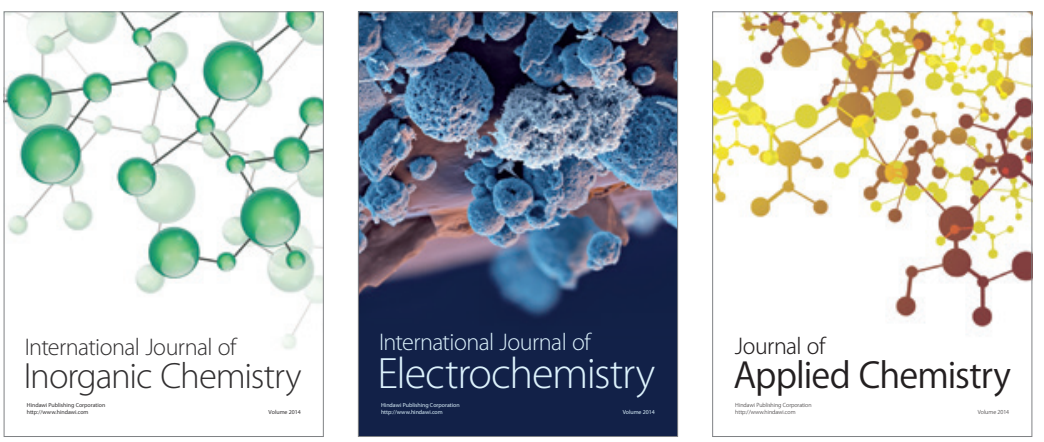

Journal of

Applied Chemistry
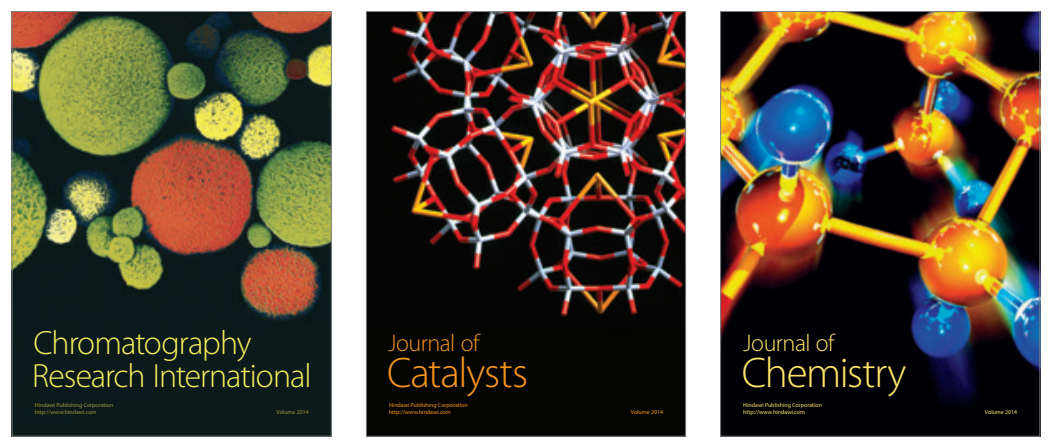
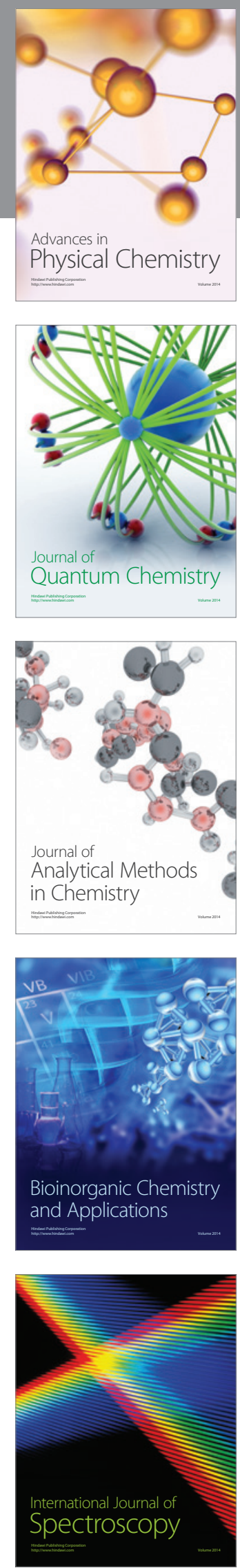